GU16 GEOLOGICAL SUREY

JLW 2 1977

Weak-Field Magnetic

Susceptibility Anisotropy

and its Dynamic Measurement

\begin{tabular}{llllll}
\hline G OLO G I A L S URVEY BULLETIN & 1418
\end{tabular}

QE 75

B9

no/418

OGS 


\section{Weak-Field Magnetic Susceptibility Anisotropy and its Dynamic Measurement}

By WILLIAM F. HANNA

$\begin{array}{llll}\text { GE L OGICAL S U R E Y B ULLETN } & 1418\end{array}$

Theory of directional properties of rock magnetic susceptibility and their measurement using a spinner magnetometer 


\section{UNITED STATES DEPARTMENT OF THE INTERIOR \\ CECIL D. ANDRUS, Secretary}

\section{GEOLOGICAL SURVEY}

V. E. McKelvey, Director

\section{Library of Congress Cataloging in Publication Data}

Hanna, William F 1938-

Weak-field magnetic susceptibility anisotropy and its dynamic measurement.

(Geological Survey bulletin ; 1418)

Bibliography: p.

Supt. of Docs. no.: I 19.3:1418

1. Rocks-Magnetic properties. 2. Magnetic susceptibility-Measurement. I. Title.

II. Series: United States. Geological Survey. Bulletin ; 1418.

QE75.B9 no. 1418 [QE431.6.M3] 557.3'08s [552'.06] 76-608101

For sale by the Superintendent of Documents, U.S. Government Printing Office Washington, D.C. 20402

Stock Number 024-001-02934-1 


\section{CONTENTS}

\begin{tabular}{|c|c|}
\hline & $g e$ \\
\hline Abstract & \\
\hline tion & \\
\hline 品 & \\
\hline Definitions of magnetic susceptibility & \\
\hline General definition & \\
\hline Definitions based on magnetization curve & \\
\hline The definition used in geophysics & \\
\hline Definitions based on other parameters & \\
\hline Units of magnetic susceptibility & \\
\hline hysical and theoretical framework of susceptibility anisotropy & \\
\hline Intrinsic vs. apparent susceptibility & \\
\hline Intrinsic susceptibility of a magnetic domain & \\
\hline Magnetocrystalline anisotropy & \\
\hline Intrinsic susceptibility of a multidomain grain & \\
\hline Apparent susceptibility of a spherical grain & \\
\hline Shape anisotropy of an ellipsoidal grain & \\
\hline Combinations of intrinsic susceptibility and shape anisotropies --- & \\
\hline Intrinsic susceptibility of a rock body & \\
\hline Minimizing anisotropy caused by rock specimen shape & \\
\hline Apparent susceptibility anisotropy of a rock formation & \\
\hline Analysis of susceptibility anisotropy of a rock specimen & \\
\hline Tensor components of linear susceptibilities & \\
\hline tric representations & \\
\hline ipal susceptibility components & \\
\hline ities & \\
\hline Anisotropy measurements using a spinner magnetometer & \\
\hline Principles of measurement & \\
\hline Independent measurement of normal susceptibility component & \\
\hline Methods of expressing anisotropy & \\
\hline Mead-type spinner magnetometer & \\
\hline Bhattacharya solution of equation of anisotropy & \\
\hline mple calculation & \\
\hline - & \\
\hline & \\
\hline
\end{tabular}

\section{ILLUSTRATIONS}

Figure 1. Idealized plot of total magnetization vs. effective magnetic field

2. Magnetization curve with Rayleigh loop _.........- 6 
Figure 3. Classification of metric units of intensity of magnetization, magnetic field, and volume magnetic susceptibility --- 14

4. Diagram illustrating tensor combinations of $\tilde{\mathbf{K}}$ and $\tilde{\mathbf{N}}-\mathbf{2}_{2}$

5. Sketch of materials possessing magnetic susceptibility anisotropy -..-_.

6. Representations of susceptibility anisotropy _........ 36

7. Diagram illustrating the relationship between rock sample

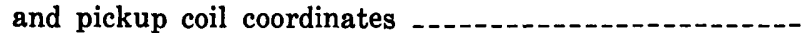

8. Diagram illustrating the relationship among the coordinate axes of the components pickup coil, rotating rock sample, and principal susceptibility

9. Diagram showing sequential order of orientations of rock sample coordinate system during the measurement process -...-..--

10. Diagram illustrating a Mead-type spinner magnetometer used for measuring susceptibility anisotropy

11. Diagram illustrating the relationship of declination and inclination to direction cosines of a principal susceptibility axis

12. Plots of maximum susceptibility plane, minor susceptibility axis, and remanent magnetization of rock sample..--

\section{TABLES}

TABLE 1. Criteria for definitions of various types of magnetic susceptibility

2. Anisotropic susceptibility data for 42 volcanic rock specimens obtained by the dynamic method of measurement.- 


\title{
WEAK-FIELD MAGNETIC SUSCEPTIBILITY ANISOTROPY AND ITS DYNAMIC MEASUREMENT
}

\author{
By William F. Hanna
}

\begin{abstract}
The apparent magnetic susceptibility, $\tilde{\mathbf{K}}_{A}$, of a uniformly magnetized body, such as a magnetic domain, mineral grain, rock unit, or rock formation, depends upon both the intrinsic magnetic susceptbility, $\tilde{\mathbf{K}}$, of the magnetic material and the demagnetizing factor, $\tilde{\mathbf{N}}$, associated with the shape of the body. This relationship, in tensor form, is
\end{abstract}

$$
\tilde{\mathbf{K}}_{\mathbf{A}}=[\tilde{\mathbf{I}}+\tilde{\mathbf{K}} \cdot \tilde{\mathbf{N}}]^{-1} \cdot \tilde{\mathbf{K}}
$$

which, for isotropic $\tilde{\mathbf{K}}$ and $\tilde{\mathbf{N}}$, reduces to the scalar relation

$$
K_{\mathrm{A}}=\frac{\mathrm{K}}{1+\mathrm{KN}} \text {. }
$$

Both $\tilde{\mathbf{K}}$ and $\tilde{\mathbf{N}}$ may be either isotropic or anisotropic. Anisotropic $\tilde{\mathbf{K}}$ of a single-domain magnetic mineral grain is associated with magnetocrystalline anisotropy. Anisotropic $\tilde{\mathbf{K}}$ of a rock specimen reflects anisotropic $\tilde{\mathbf{K}}$ and (or) $\tilde{\mathbf{N}}$ of constituent magnetic mineral grains. Anisotropic $\tilde{\mathbf{N}}$, associated with shape anisotropy of a body, becomes isotropic as the shape of the body becomes equidimensional. Laboratory measurements of the anisotropic $\tilde{\mathbf{K}}_{A}$ of an equidimensional rock specimen can be directly related to the anisotropic $\tilde{\mathbf{K}}$ of the rock. This anisotropic $\tilde{\mathbf{K}}$ of the rock may, under certain conditions, give information about shape anisotropiesi assocated with alinements of magnetic mineral grains or magnetic domains within individual grains of the rock.

The weak-field apparent susceptibility anisotropy of a uniformly magnetized rock specimen can be dynamically measured by means of a spinner magnetometer if interfering second-harmonic signals due to remanent dipole moments are cancelled and if effects due to electrical conductivity and remanent quadrupole moments are sufficiently small. Complete determination of the six susceptibility components, from which magnitudes and directions of principal axes can be computed, requires an additional measurement of one normal susceptibility component using a stationary-sample bridge. Measurements of 106 miscellaneous volcanic rock specimens, by means of a Mead-type spinner magnetometer, and computations based on procedures of $\mathrm{P}$. K. Bhattacharya, indicate that 42 specimens have measurable anisotropies and that 30 of these specimens have susceptibilities that are sufficiently linear to be represented by ellipsoids or spheroids. 


\section{INTRODUCTION}

The anisotropy of magnetic susceptibility of a rock sample may be adequately measured by means of a spinner magnetometer provided that the induced magnetization is related linearly to the magnetic field within the rock sample and provided that unwanted signals and noise can be eliminated in the measurement process. This dynamic process of measuring anisotropy, introduced by Bhattacharya (1950), has received relatively little attention because of the general inaccessibility of this dissertation in the literature. The main attraction of the dynamic method is that the same apparatus routinely used to measure remanent magnetization of samples can, with minimal alteration, be used to measure susceptibility anisotropy. For rocks not satisfying the linearity condition between magnetization and magnetic field and for those generating unwanted signals or noise that cannot be eliminated, more sophisticated devices (Graham, 1967) ${ }^{1}$ must be used to measure the associated relatively complex anisotropy.

After the work of Bhattacharya (1950), references to the dynamic method of measurement appeared in a report by Howell, Martinez, and Statham (1958) on magnetic properties of sedimentary and metamorphic rocks; in dissertations of Noltimier $(1965 ; 1967)$ and Hanna $(1965)$ on magnetic properties of redbeds and volcanic rocks, respectively; and in the instruction manuals by commercial manufacturers of spinner magnetometers, such as Princeton Applied Research Corporation and Schonstedt Instrument Company. However, detailed accounts of the measurement and computation of anisotropy by means of a spinner magnetometer are not generally available. This lack of a comprehensive published account has become evident during the past 10 years from the numerous requests received for information about the dynamic method of measurement. This report is intended to provide an overview of the physical and mathematical framework of magnetic susceptibility and associated anisotropy, its measurement by means of a spinner magnetometer developed by Professor Judson Mead of Indiana University, its computation by the technique of the late Dr. P. K. Bhattacharya, and some examples of measurements and results for volcanic rocks.

\footnotetext{
1 The most highly refined technique for measuring susceptibility anisotropy of a rotating rock sample was developed over a 12-year period by the late John W. Graham, a pioneer in this type of geophysical investigation. Graham is credited by Bhattacharya (1950) for his assistance in the original study involving the dynamic method.
} 


\section{ACKNOWLEDGMENT}

I first became interested in problems of weak-field susceptibility anisotropy in graduate research directed by Professor Judson Mead at Indiana University.

\section{DEFINITIONS OF MAGNETIC SUSCEPTIBILITY}

\section{GENERAL DEFINITION,}

When a substance, such as a rock, is exposed to a magnetic field, it acquires magnetization, which is said to be caused by the field. The functional relationship between this magnetization and the effective magnetic field at a point within the substance is defined in terms of the magnetic susceptibility. If $\mathrm{H}_{\text {eff }}$ is the magnitude of the effective magnetic field applied in a particular direction relative to coordinate axes of the material, $\mathrm{K}\left(\mathrm{H}_{\mathrm{eff}}\right)$ is the magnitude of the magnetic susceptibility, and $J\left(\mathrm{H}_{\mathrm{eff}}\right)$ is the magnitude of the magnetization caused by $\mathrm{H}_{\text {eff }}$, the defining relation is

$$
J\left(\mathrm{H}_{\text {eff }}\right)=\left[\mathrm{K}\left(\mathrm{H}_{\mathrm{eff}}\right)\right] \mathrm{H}_{\mathrm{eff}} \text {. }
$$

In equation $1, \mathrm{H}_{\text {eff }}$ is an independent variable and the component of an axial vector, $\mathrm{J}\left(\mathrm{H}_{\text {eff }}\right)$ is a dependent variable of $\mathrm{H}_{\text {eff }}$ and the component of an axial vector, and $\mathrm{K}\left(\mathrm{H}_{\mathrm{eff}}\right)$ is a multivalued function of $\mathrm{H}_{\text {eff }}$ and the component of a tensor. The magnetic susceptibility is an aptly named quantity, for it expresses the extent to which a substance is susceptible of acquiring magnetization in the presence of a magnetic field. Because the general definition of magnetic susceptibility given by equation 1 contrasts slightly with a more restrictive definition ordinarily used in geophysics, we shall consider several susceptibility definitions in terms of a plot of magnetization versus effective magnetic field.

\section{DEFINITIONS BASED ON MAGNETIZATION CURVE}

The relationship between magnetization and effective magnetic field at a point within a ferromagnetic substance is shown in figure 1. In the diagram, we consider the magnetization to be total magnetization (combination of remanent and induced magnetization) and the substance to be homogeneous, at constant temperature and pressure, and in an initially demagnetized or neutral state. From its zero value at $O, \mathrm{~J}\left(\mathrm{H}_{\text {eff }}\right)$ increases nonlinearly along the dashed line to its saturation value at $B$, where further increase in $\mathrm{H}_{\text {eff }}$ results in negligibly small, if any, increase of $\mathrm{J}\left(\mathrm{H}_{\text {eff }}\right)$. If $\mathrm{H}_{\text {eff }}$ at $\mathrm{H}_{B}$ is decreased and reversed to its value at $\mathrm{H}_{F}, \mathrm{~J}\left(\mathrm{H}_{\mathrm{eff}}\right)$ decreases along the path $B C D E^{\prime} E F$ to its saturation 


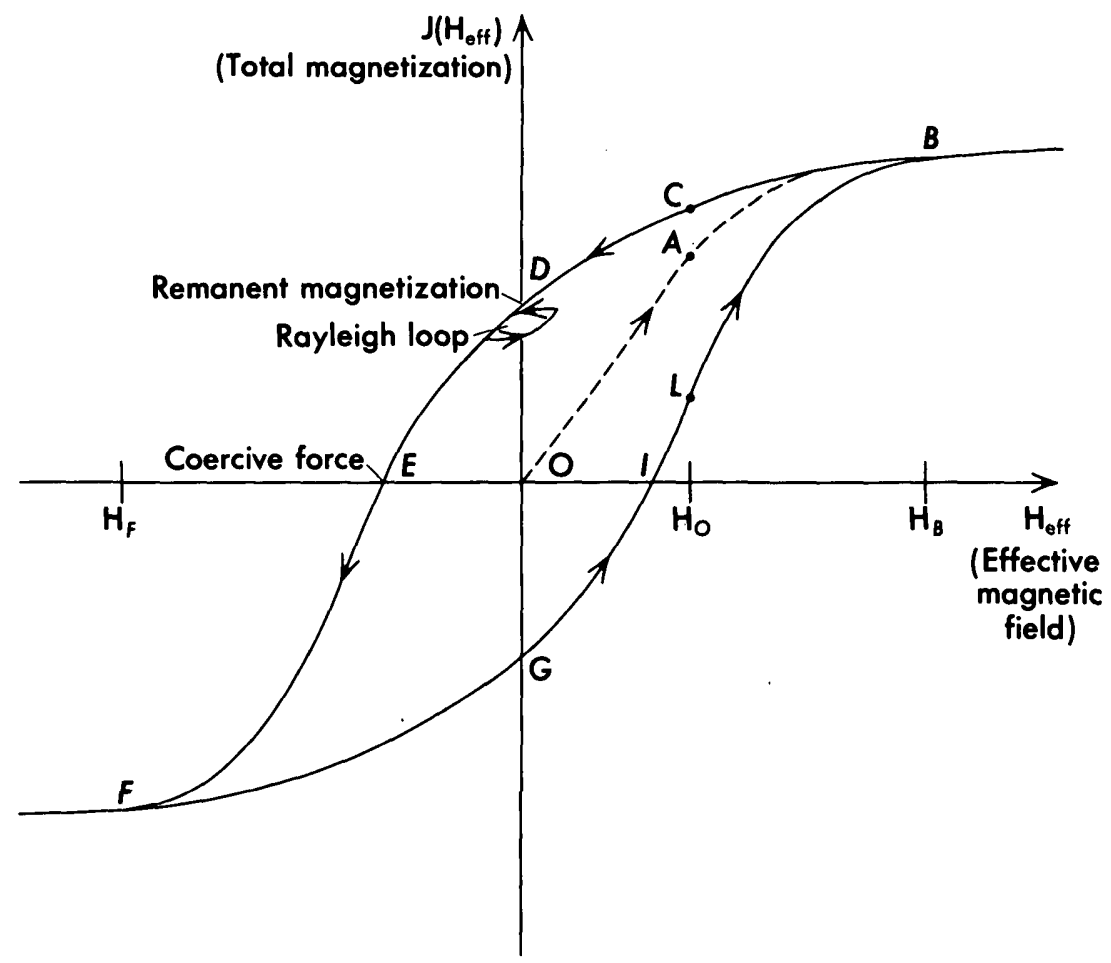

FIGURE 1.-Idealized plot of total magnetization vs. effective magnetic field showing initial magnetization curve $O A B$ and hysteresis loop BCDEFGILB. Magnetization curve serves to define initial, differential, reversible, irreversible, total, and maximum magnetic susceptibility.

value in the opposite direction. Another reversal and increase of $\mathrm{H}_{\text {eff }}$ causes $\mathrm{J}\left(\mathrm{H}_{\text {eff }}\right)$ to increase along the path $F G I L B$ to saturation again. Subsequent fluctuations of $\mathrm{H}_{\text {eff }}$ between the values $\mathrm{H}_{B}$ and $\mathrm{H}_{E}$ drive $\mathrm{J}\left(\mathrm{H}_{\text {eff }}\right)$ repeatedly around the path $B C D E^{\prime} E F G I L B$, termed a hysteresis (from the Greek word meaning "to be behind") loop, because the decrease or increase of $\mathrm{J}\left(\mathrm{H}_{\text {eff }}\right)$ to zero takes place after the decrease or increase of $\mathrm{H}_{\text {eff }}$ to zero. The magnetization $O D$ or $O G$ is termed the remanent or permanent magnetization because it exists in the absence of a magnetic field, once the material is driven from its neutral state. The magnetic field $O E$ or $O I$ is termed the coercive force or coercive field because it is the field required to coerce or force the magnetization from its remanent value to zero. The hysteresis loop serves as a basis for defining several magnetic-susceptibility terms used in the literature, such as initial, reversible, irreversible, total, and maximum magnetic susceptibility (see, for example, Stoner, 1934; Nagata, 1961; Chikazumi, 1964 ; Standley, 1972). 
In terms of the hysteresis loop of figure 1, the initial susceptibility is the slope of the initial magnetization curve $O A B$ at the origin $O$. The differential susceptibility is defined as $\frac{d}{d \mathrm{H}_{\text {eff }}}\left[\mathrm{J}\left(\mathrm{H}_{\text {eff }}\right)\right]$ at any point on the initial magnetization curve, assuming $\mathrm{H}_{\text {eff }}$ is increasing. The reversible susceptibility is defined as the incremental change in $\mathrm{J}\left(\mathrm{H}_{\text {eff }}\right)$ per incremental change in $\mathrm{H}_{\text {eff }}$ as $\mathrm{H}_{\text {eff }}$ is decreasing. The irreversible susceptibility is the algebraic difference between the differential and reversible susceptibilities at a point on the curve. The total magnetic susceptibility is defined as the ratio of $J\left(\mathrm{H}_{\text {eff }}\right)$ to $\mathrm{H}_{\text {eff }}$ at any point on the initial magnetization curve, and the maximum susceptibility is the maximum value of total susceptibility. The multivalued characteristic of $\mathrm{K}\left(\mathrm{H}_{\mathrm{eff}}\right)$ is evident from the difference in slope of curves at points $L, A$, and $C$, corresponding to $\mathrm{H}_{\text {eff }}=\mathrm{H}_{o}$. The vertical separation of these points manifests the multivalued characteristic of $\mathrm{J}\left(\mathrm{H}_{\text {eff }}\right)$.

Of special interest in geophysical work are magnetic susceptibilities and magnetizations corresponding to weak magnetic fields on the hysteresis loop. Because the effective magnetic fields in crustal rocks near the earth's surface are generally less than 1 oersted in magnitude, the magnetic state of most rocks is close to point $D$ on the hysteresis loop of figure 1. If, for example, the magnetic state of a rock is represented by point $E^{\prime}$ (fig. 2), corresponding to a slightly negative effective magnetic field such as a naturally occurring demagnetizing field, an increase in $\mathrm{H}_{\text {eff }}$ will not necessarily drive $\mathrm{J}\left(\mathrm{H}_{\text {eff }}\right)$ along the path $E^{\prime} D$ to the point of remanent magnetization. Instead, $\mathrm{J}\left(\mathrm{H}_{\mathrm{eff}}\right)$ may be driven along the lower branch of an elliptical Rayleigh loop (Rayleigh, 1887) to point $E^{\prime \prime}$, whereupon it returns along the upper branch to point $E^{\prime}$, provided that $\mathrm{H}_{\text {eff }}$ is again reversed. This nonlinear change in magnetization for a small change in magnetic field results in a small change in remanent magnetization but no change in the overall magnetic state of the material given by point $E^{\prime}$. The reversible susceptibility associated with the small Rayleigh loop is commonly taken to be the major axis of the loop, shown by a dashed line in figure 2 .

If the change in magnetic field is kept sufficiently small, say, to less than 1 oersted, the magnetization of most crustal rocks undergoes a linear change. For example. if $\mathrm{H}_{\text {eff }}$ fluctuates between the values $\mathrm{H}_{D^{\prime}}$ and $\mathrm{H}_{D^{\prime \prime}}$ in figure $2, \mathrm{~J}\left(\mathrm{H}_{\text {eff }}\right)$ will be repeatedly driven along the linear path segment $D^{\prime} D D^{\prime \prime}$. The slope of the linear segment $D^{\prime} D D^{\prime \prime}$ determines the reversible weak-field mag- 


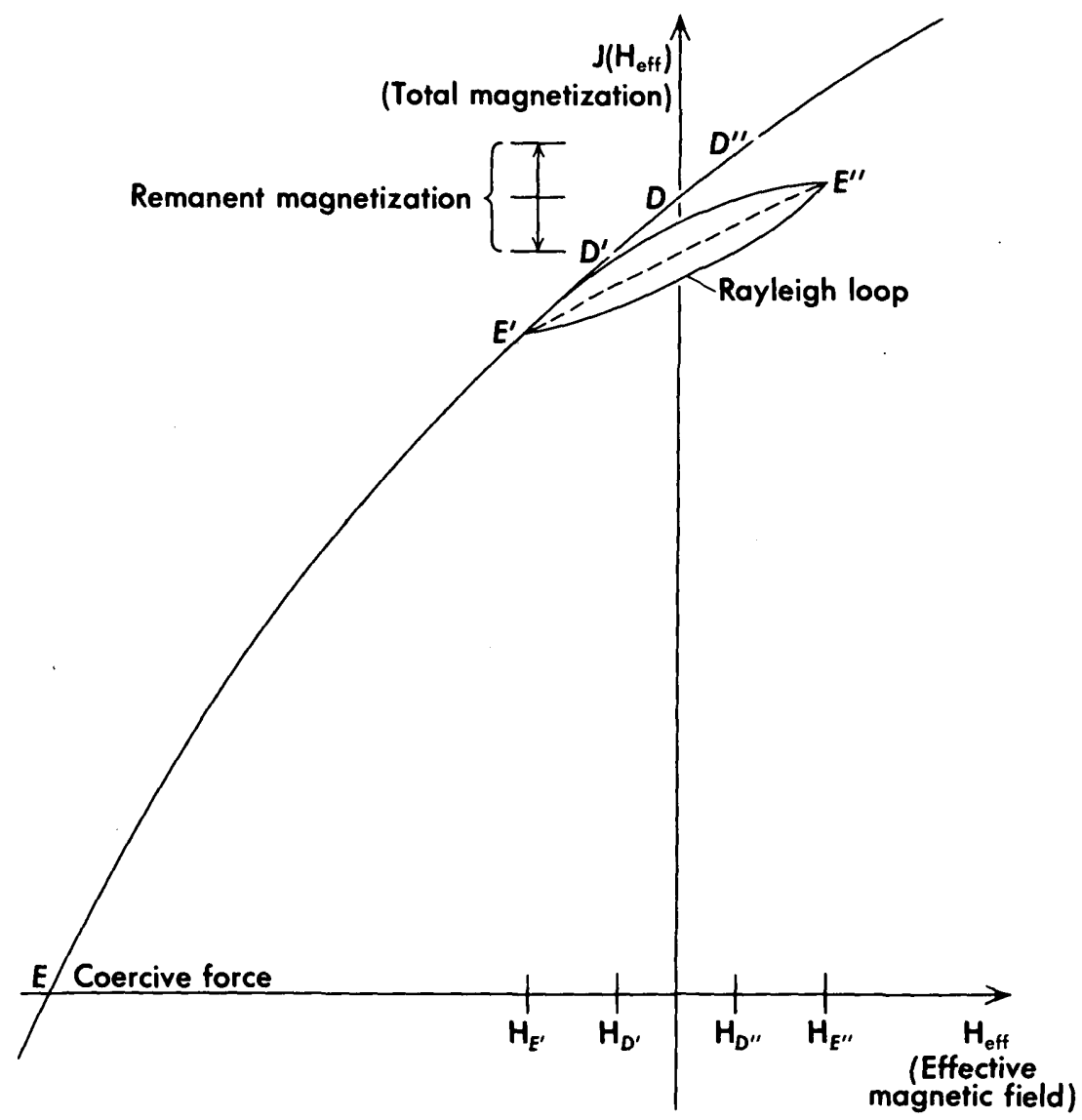

FIGURE 2.-Part of magnetization curve shown in figure 1, showing Rayleigh loop. Dashed line $E^{\prime} E^{\prime \prime}$ is an approximate representation of a linear reversible magnetic susceptibility.

netic susceptibility, and the increment of magnetization associated with the nonzero magnetic field is linear-induced magnetization. It is this linear-induced magnetization that is ordinarily considered in the definition of magnetic susceptibility as used in solidearth geophysics.

\section{THE DEFINITION USED IN GEOPHYSICS}

In much geophysical work involving the interpretation of magnetic anomaly data in tectonic mapping, petroleum exploration, and ore prospecting, the magnetic susceptibility is considered to be not only a linear quantity but also a single constant. This assumption is justified in studies involving most sedimentary and metamorphic rocks and most plutonic and volcanic rocks of felsic 
to moderately mafic composition. If $\mathrm{J}$ is the magnitude of induced magnetization, $\mathrm{H}_{\text {eff }}$ is the magnitude of effective magnetic field, and $\mathrm{K}$ is the magnitude of intrinsic magnetic susceptibilty of the rock, we may write

$$
\mathrm{J}=\mathrm{KH}_{\text {eff. }}
$$

In other geophysical work, such as studies of anomalies associated with highly magnetic mafic rocks or iron ores, analyses of the fabric of magnetic mineral grains within a rock, and investigations of the possible influence of induced magnetization on the acquisition of remanent magnetization, the magnetic susceptibility may be considered a linear quantity but not a single constant. Instead, the linear magnetic susceptibility in weak magnetic fields must generally be expressed by six constants. For these investigations, the magnetic susceptibility may be defined by the relation

$$
\overrightarrow{\boldsymbol{J}}=\tilde{\mathbf{K}} \cdot \overrightarrow{\boldsymbol{H}}_{\text {eff, }}
$$

where $\overrightarrow{\boldsymbol{J}}$ is the induced magnetization vector, $\tilde{\mathbf{K}}$ is the intrinsic magnetic susceptibility tensor, and $\overrightarrow{\boldsymbol{H}}_{\text {eff }}$ is the effective magnetic field vector. Because $\tilde{\mathbf{K}}$ operates on $\overrightarrow{\boldsymbol{H}}_{\text {eff }}$ to produce $\overrightarrow{\boldsymbol{J}}, \tilde{\mathbf{K}}$ is known as a linear vector operator. This operator is also a second-rank tensor, a quantity having nine components, as shown by expanding equation 3 in terms of cartesian components

$$
\begin{aligned}
& J_{x}=K_{x x}\left(H_{\text {eff }}\right)_{x}+K_{x y}\left(H_{\text {eff }}\right)_{y}+K_{x z}\left(H_{\text {eff }}\right)_{z}, \\
& J_{y}=K_{y x}\left(H_{\text {eff }}\right)_{x}+K_{y y}\left(H_{\text {eff }}\right)_{y}+K_{y z}\left(H_{\text {eff }}\right)_{z},
\end{aligned}
$$

and

$$
J_{z}=K_{z x}\left(H_{\text {eff }}\right)_{x}+K_{z y}\left(H_{\text {eff }}\right)_{y}+K_{z z}\left(H_{\text {eff }}\right)_{z} .
$$

Thus, an effective magnetic field applied along, say, the $y$ direction produces an induced magnetization not only along the $y$ direction but also along the $x$ and $y$ directions.

The nine susceptibility components in equations 4 are reduced to six components as a consequence of the law of conservation of energy. This law requires that

$$
K_{x y}=K_{x y}, K_{x z}=K_{z x} \text {, and } K_{y z}=K_{z y} \text {. }
$$

Relations 5 indicate that, for example, the magnetization induced along the $z$ direction by a magnetic field along the $x$ direction is equal to the magnetization induced along the $x$ direction by a field of the same strength along the $z$ direction. Rocks for which 


$$
K_{x y}=K_{x z}=K_{y z}=0
$$

and

$$
K_{x x}=K_{y y}=K_{z z}
$$

are said to have an isotropic magnetic susceptibility. Rocks for which relations 6 and 7 do not hold are said to possess an anisotropic magnetic susceptibility.

\section{DEFINITIONS BASED ON OTHER PARAMETERS}

In addition to susceptibilities conveniently defined in terms of the hysteresis loop and to those previously mentioned which have been adopted for use in geophysical research, many others commonly appear in the literature. A list of many of these special definitions of susceptibility, together with criteria for the definitions and comments about distinctive characteristics of each susceptibility, is presented in table 1. Susceptibility terms used in the present report include those described as ferromagnetic, volume, crystalline, domain, mineral grain, rock, rock formation, intrinsic, apparent, reversible, linear, constant, principal, weakfield, dynamic sample, stationary sample, A. C., and bulk, as noted in table 1. Anisotropic susceptibility treated throughout the report is considered to be linear, a characteristic which implies that the induced magnetization is related to only the first power of the effective magnetic field.

\section{UNITS OF MAGNETIC SUSCEPTIBILITY}

When considering various expressions of units that have been applied to magnetic susceptibility, it is important to note that metric systems of magnetic units are commonly grouped in three ways (Kennelly, 1936; Stratton, 1941; Smythe, 1968). First, they belong to either a centimetre-gram-second (CGS) system or to a metre-kilogram-second (MKS) system according to the mechanical units used. Second, they belong to either a classical or practical system depending upon the relative magnitudes of certain units, such as those of electric current or charge. Third, they belong to either the rationalized or unrationalized system depending upon whether factors of $4 \pi$ appear in certain expressions, such as those of current and charge. The CGS classical units may be subdivided into electromagnetic, electrostatic, or a mixture of these, according to arbitrarily chosen relationships of electrical permittivity and magnetic permeability to the speed of light in a vacuum.

The most commonly used systems of magnetic units are shown in the classification diagram of figure 3 . The values given in figure 
3 are all based on unit (value of one) magnitudes for these quantities in the SI metric system.

In rock-magnetic research, the Gaussian system has been used most widely. Volume magnetic susceptibility in this system has been traditionally expressed as $\mathrm{emu} / \mathrm{cm}^{3}$, an ambiguous designation identical with that used for volume intensity of magnetization in the same system of units. As an alternative to the emu/ $\mathrm{cm}^{3}$ Gaussian unit for susceptibility, the dimensionally equivalent gauss/oersted has been adopted by Collinson, Creer, and Runcorn (1967), even though it is $4 \pi$ times larger than the traditional Gaussian unit. The gauss/oersted is numerically equivalent to one SI unit.

As use of the internationally adopted SI units increases in geophysical work, the most important conversion factor for units of magnetic susceptibility will be

and

$$
1 \frac{\mathrm{emu}}{\mathrm{cm}^{3}}=4 \pi(\mathrm{SI})
$$

$$
1(\mathrm{SI})=7.96 \times 10^{-2} \frac{\mathrm{emu}}{\mathrm{cm}^{3}} .
$$

One SI unit of susceptibility (actually a dimensionless quantity) is representative of a susceptibility value characteristic of many types of highly magnetic coarse-grained rocks, such as gabbro, magnetite sandstone, and magnetite-rich iron ore.

\section{PHYSICAL AND THEORETICAL FRAMEWORK OF SUSCEPTIBILITY ANISOTROPY}

\section{INTRINSIC VS. APPARENT SUSCEPTIBILITY}

Because the induced magnetization of a finite magnetic body is measured upon application of a carefully measured magnetic field applied externally to the body, this magnetization can be conveniently referred to the external magnetic field rather than to the internal effective magnetic field. This convenience of expression leads to the definition of apparent magnetic susceptibility,

$$
\overrightarrow{\boldsymbol{J}}=\tilde{\mathbf{K}}_{\boldsymbol{A}} \cdot \overrightarrow{\boldsymbol{H}}_{\text {ext }},
$$

where $\overrightarrow{\boldsymbol{J}}$ is the induced magnetization vector, $\overrightarrow{\boldsymbol{H}}_{\text {ext }}$ is the external magnetic field vector, and $\widetilde{\mathbf{K}}_{A}$ is the apparent magnetic susceptibility tensor. This definition contrasts with expression 3 , defining intrinsic magnetic susceptibility,

$$
\overrightarrow{\boldsymbol{J}}=\tilde{\mathbf{K}} \cdot \overrightarrow{\boldsymbol{H}}_{\text {eff }},
$$




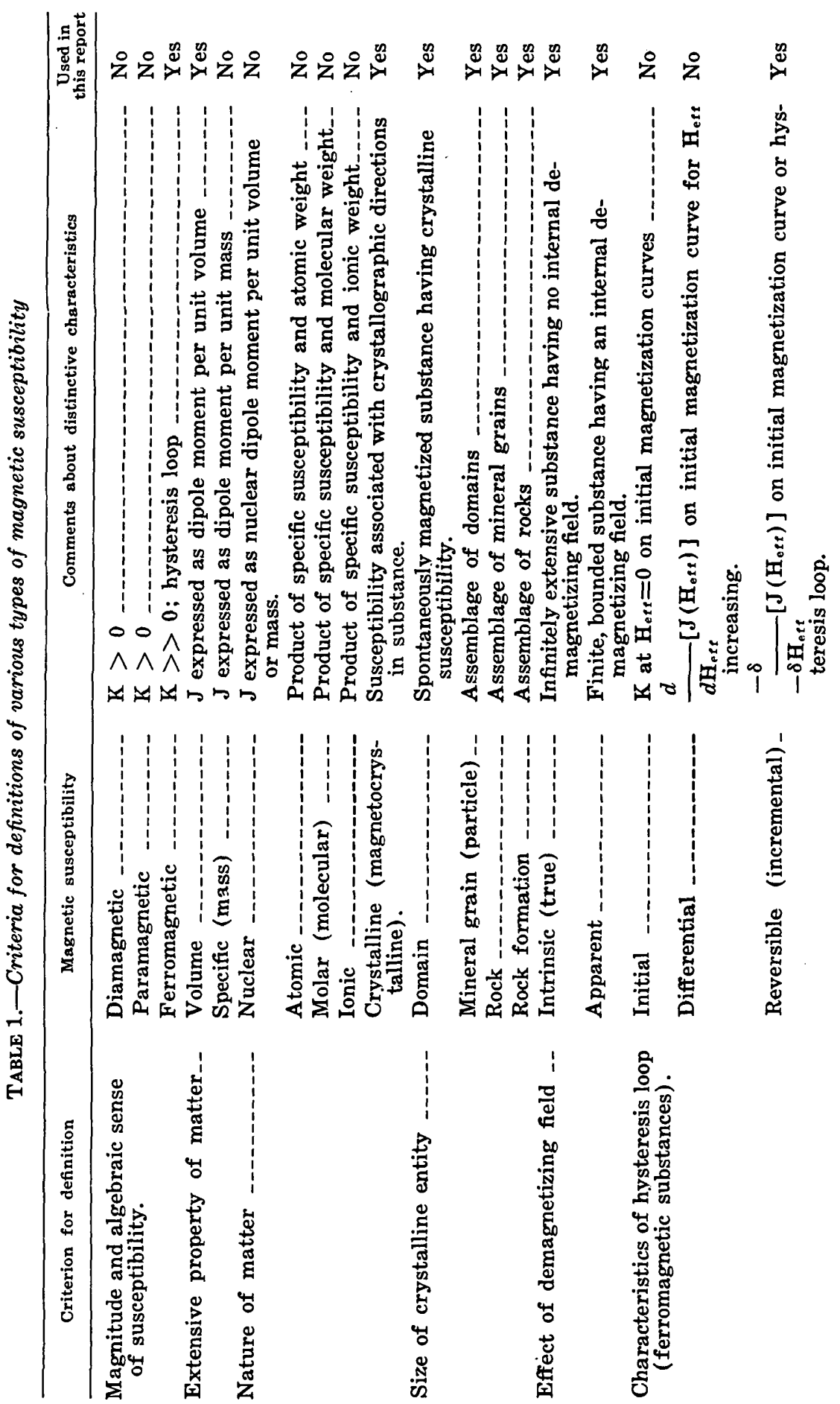




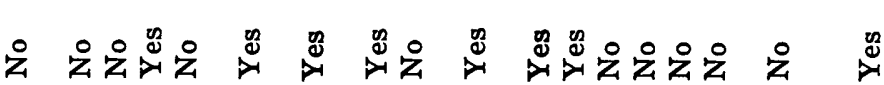

苛

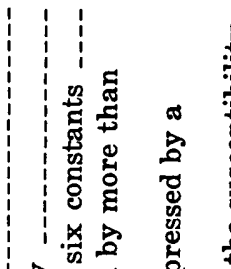

胥

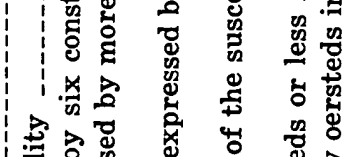

:

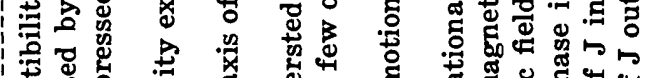

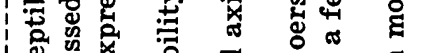

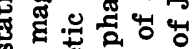

ฐ

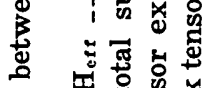

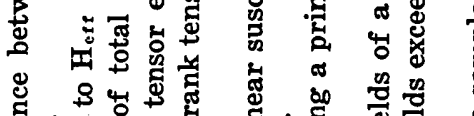

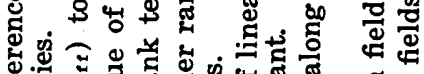

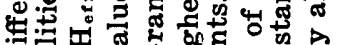

..

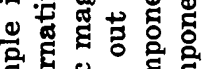

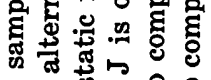

$\cong$

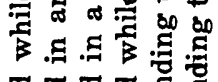

\%

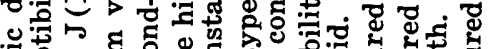

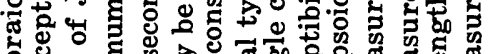

to

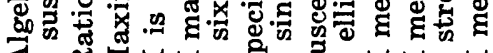

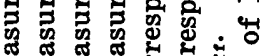

(.

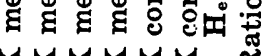

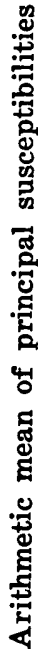

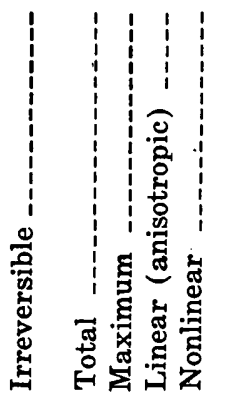

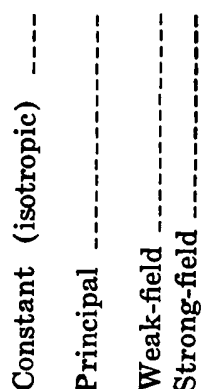

है

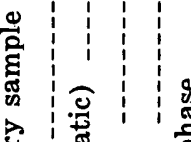

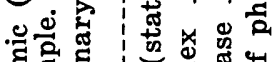

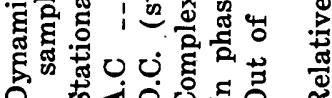
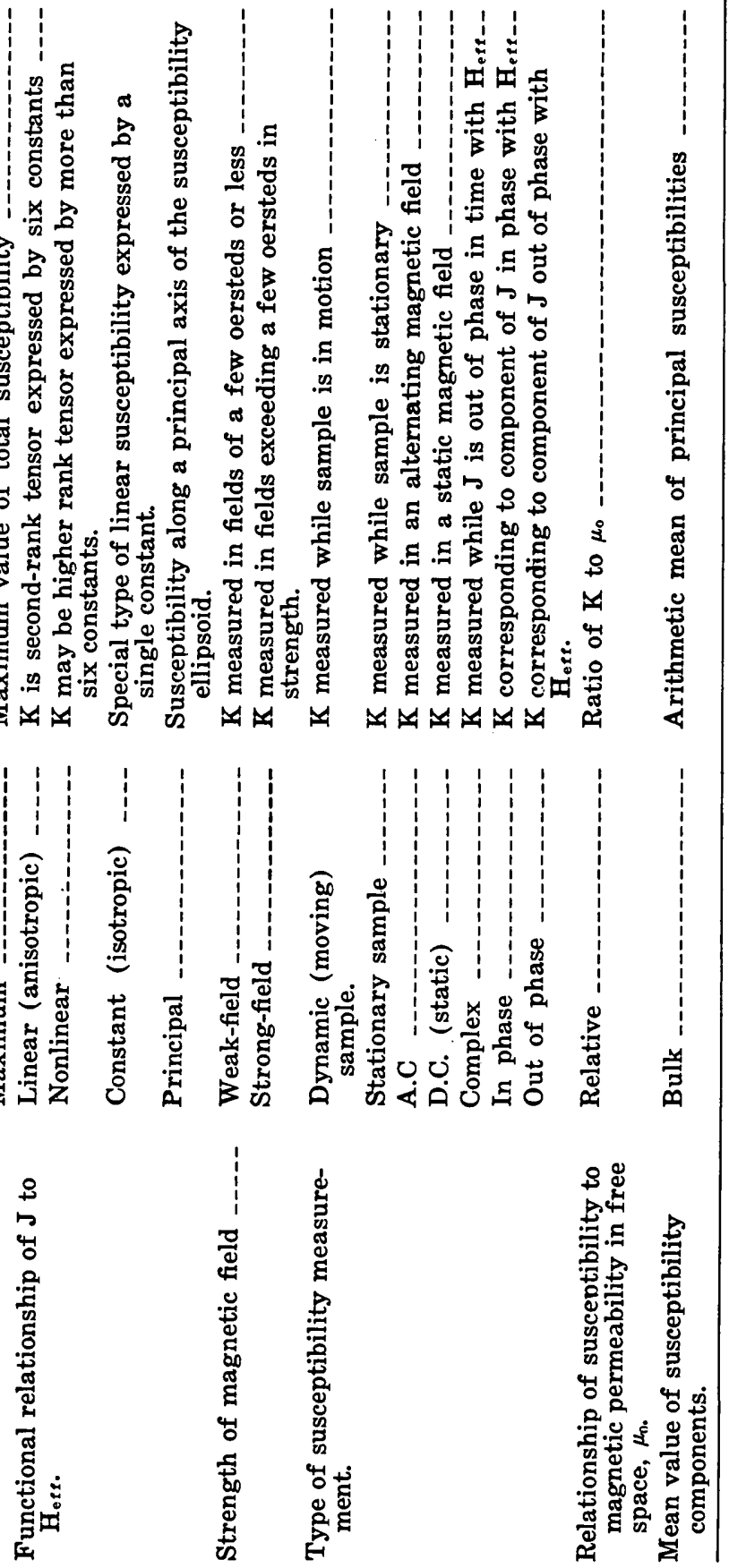


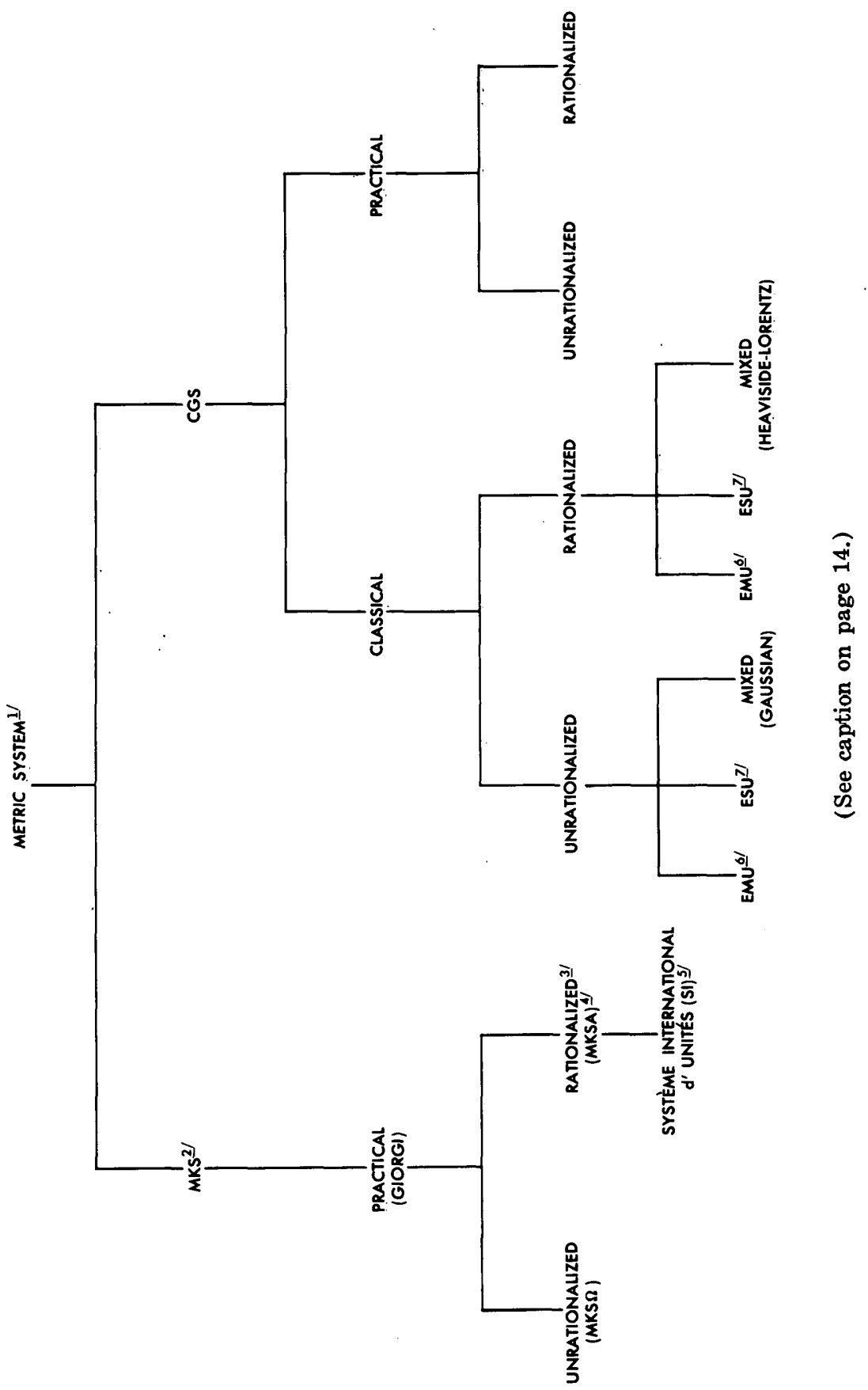




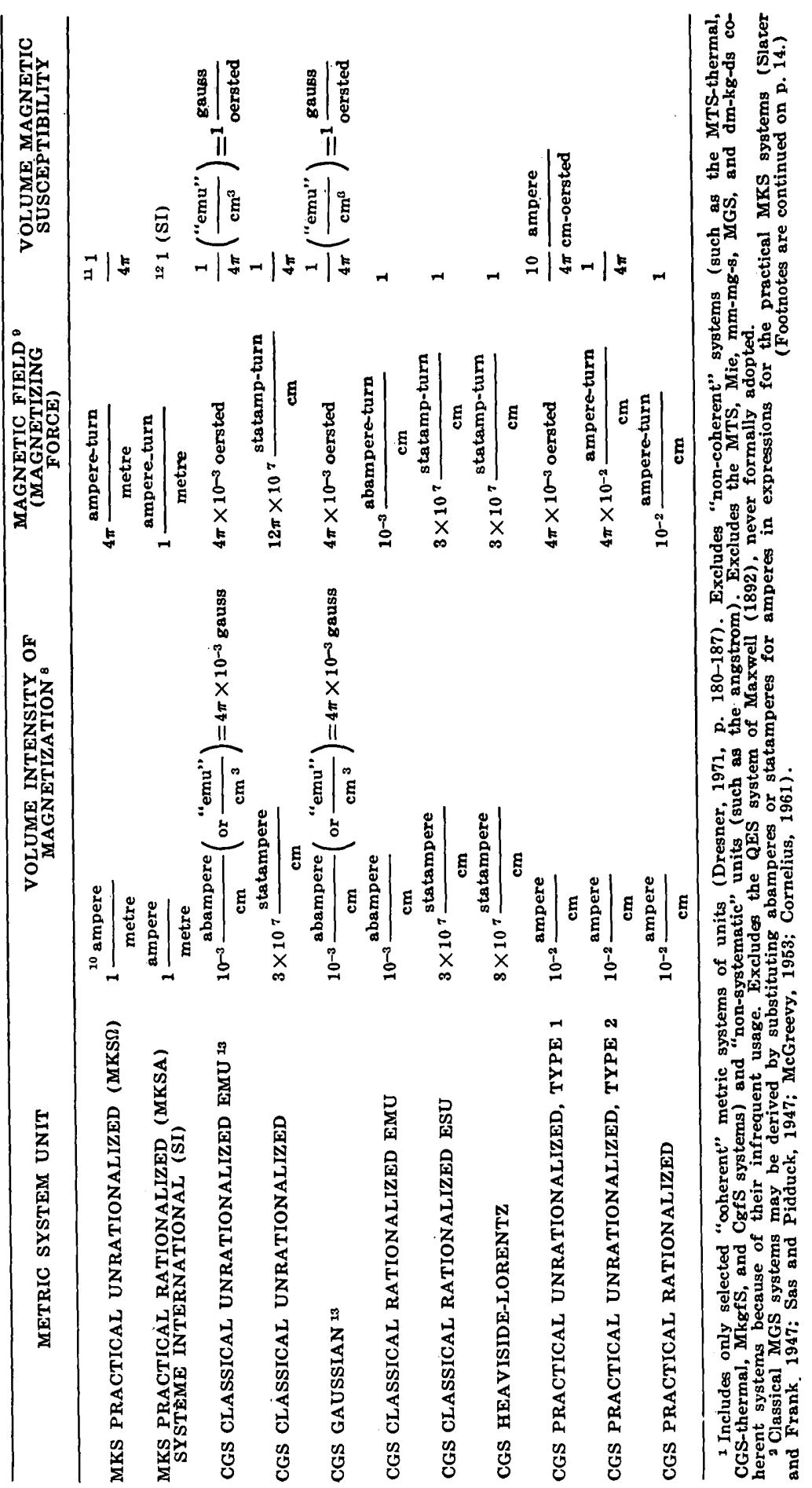




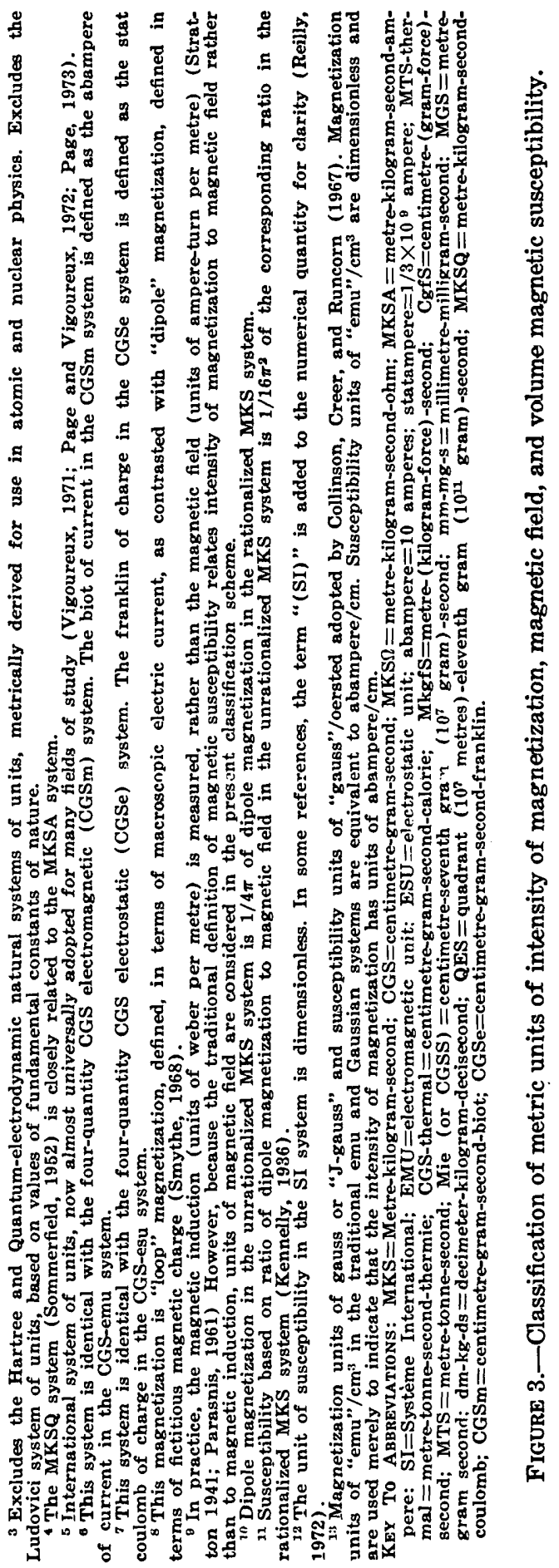


where, as previously noted, $\overrightarrow{\boldsymbol{H}}_{\text {eff }}$ is the effective magnetic field vector inside the body and $\tilde{\mathbf{K}}$ is the intrinsic magnetic susceptibility.

The apparent magnetic susceptibility is intimately related to the shape of the body and to an internal demagnetizing field which is produced within the body upon application of the external magnetic field. The intrinsic magnetic susceptibility, in contrast, does not depend upon the shape of the body. The intrinsic susceptibility is a property of the magnetic material itself which may be considered to extend continuously without bounds. The intrinsic susceptibility is ordinarily computed from the measured apparent susceptibility, given information about the shape of the magnetic body.

Both intrinsic and apparent magnetic susceptibility may be either isotropic or anisotropic. If the intrinsic susceptibility is isotropic, the apparent susceptibility can be isotropic only if the body is equidimensional. If the intrinsic susceptibility is anisotropic, the apparent susceptibility will in general be anisotropic also, whether or not the body is equidimensional. Under highly fortuitous circumstances, the apparent susceptibility of a nonequidimensional body possessing anisotropic intrinsic susceptibility may itself be isotropic, provided that the anisotropy associated with the shape of the body cancels the intrinsic anisotropy. In practice, measurements of susceptibility anisotropy are ordinarily restricted to equidimensional specimens so that observed apparent susceptibility anisotropy can be directly related to intrinsic susceptibility anisotropy.

The finite body to which the concepts of intrinsic and apparent susceptibility apply may be a mineral grain consisting of one or more magnetic domains, ${ }^{2}$ a rock body (a naturally occurring rock unit or a specimen cut from such a unit) composed of magnetic mineral grains, or a rock formation consisting of magnetic rock bodies. The intrinsic susceptibility anisotropy of any of these bodies depends upon the intrinsic susceptibilities and shapes of magnetic constituents that make up the body.

For example, if the finite body consists of a single magnetic domain, the intrinsic susceptibility of the domain is associated exclusively with the crystalline material of the domain. If the finite body is a multidomain magnetic mineral grain, the intrinsic susceptibility of the grain is associated with both the intrinsic

2 A magnetic domain is a spontaneously magnetized region generally having dimensions of a few hundredths of a micron, which forms all or part of a magnetic mineral grain (see, for example, Bates, 1961; Chikazumi, 1964). 
susceptibilities of the domains and the shapes of the domains. If the finite body is a rock unit, the intrinsic susceptibility of the rock unit is associated with both the intrinsic susceptibilities of the grains and the shapes of the grains. If the finite body is a rock formation composed of a group of rock units, the intrinsic susceptibility of the rock formation is associated with both the intrinsic susceptibilities of the rock units and the shapes of the rock units.

\section{INTRINSIC SUSCEPTIBILITY OF A MAGNETIC DOMAIN}

The smallest physical entity to which the concept of magnetic susceptibility is generally applied in rock magnetic studies is the magnetic domain. ${ }^{3}$ In the absence of an applied magnetic field, the spontaneous magnetization of a domain is alined parallel to one of several crystallographic directions called easy magnetization directions of the magnetic mineral. For example, in magnetite, the axis of easiest magnetization is perpendicular to the octahedral plane, whereas the axis of most difficult magnetization is perpendicular to the hexahedral plane (Nagata, 1961). If the crystalline material of a magnetic domain is subjected to an applied magnetic field, the spontaneous magnetization will tend to rotate toward the direction of the applied field. If the applied field is sufficiently weak, the magnetization will reversibly rotate to its initial direction upon removal of the applied field.

\section{MAGNETOCRYSTALLINE ANISOTROPY}

Because the crystalline material constituting a magnetic domain is more easily magnetized along some crystallographic directions than along others, the domain is said to possess a magnetocrystalline anisotropy of magnetic susceptibility. Thus, the magnetization of a domain composed of a particular magnetic mineral can be characterized by a separate curve, such as that shown in figure 1 , for each direction of applied magnetic field relative to the crystallographic axes of the mineral. According to studies of Uyeda, Fuller, Belshé, and Girdler (1963), a weakfield magnetocrystalline anisotropy is negligibly small in cubic titanomagnetites but larger in rhombohedral ilmenohematites. These observations indicate that the initial magnetization curves, $O A B$ (fig. 1), for various applied field directions in titanomagnetites coincide or overlap for small values of applied field but di-

\footnotetext{
3 Domains occur in a broad class of ferromagnetic materials, including true ferromagnetic, antiferromagnetic, and ferrimagnetic minerals referred to in this report as "magnetic min. erals." The atomic and subatomic origins of magnetic susceptibility of all materials. including those that are diamagnetic and paramagnetic, are subjects of quantum mechanics, beyond the intended scope of this report.
} 
verge or splay for larger values of applied field. The corresponding curves for ilmenohematites diverge or splay for relatively small values of applied field.

The geometry and relative mathematical complexity of magnetocrystalline anisotropy in a material is dependent upon the crystal class of the material. Studies of crystal symmetry using time- as well as space-coordinate transformations indicate the existence of 90 magnetocrystalline classes of materials having 1,651 space groups, in contrast to the 32 crystal classes and 230 space groups corresponding to space-coordinate transformations alone (Birss, 1964 ; Bhagavantam, 1966; Billings, 1969). Although the large number of magnetocrystalline classes places many restrictions on physical properties of a material not expressible by second-rank tensors, fewer restrictions are placed on second-rank tensor properties. The constraints posed by Neumann's Principle (see, for example, Nye, 1960, p. 20) merely require that the tensor geometry corresponding to magnetic susceptibility have a symmetry at least as high as the crystal symmetry of the material and that certain crystallographic axes in the material coincide with particular directions of the tensor property. The main crystallographic systems represented by naturally occurring magnetic minerals are the cubic system (magnetite-ulvöspinel series and related inverse-spinel minerals) and the hexagonal system (ilmenite-hematite series and pyrrhotite).

\section{INTRINSIC SUSCEPTIBILITY OF A MULTIDOMAIN GRAIN}

In a typical magnetic mineral grain composed of two or more domains, distinct walls (called "Bloch walls" (Kittel, 1953, p. 183)) separating domains of oppositely directed spontaneous magnetization (called $180^{\circ}$ walls) tend to aline parallel to easy directions of magnetization. If a magnetic field is applied parallel to the easy direction, the $180^{\circ}$ walls move laterally; domains having spontaneous magnetizations parallel to the applied field grow at the expense of domains having magnetizations antiparallel to the applied field. If a magnetic field is applied perpendicular to the easy direction, the $180^{\circ}$ walls remain stationary, and the spontaneous magnetizations of the domains rotate toward the applied field direction (Stacey, 1961). Ordinarily, applied fields required to produce a given amount of magnetization by magnetization rotation are larger than those required to produce the same amount of magnetization by $180^{\circ}$ wall displacement. In relatively weak applied fields of a few oersteds, $180^{\circ}$ wall movements 
and spontaneous magnetization rotations are found to be reversible upon cancellation of the applied field.

The development of $180^{\circ}$ walls (and walls having other angular relationships between adjacent domain magnetizations) in a magnetic mineral grain depends upon the shapes of the domains, which, in turn, relate to amounts of magnetostatic energy developed at the margins of the domains. The ease of movements of domain walls upon application of a magnetic field are thus also related to the external shapes of the domains. The ease of rotations of magnetization upon application of a field are, however, not dependent upon the shapes of domains, but are dependent only upon the magnetocrystalline energy of the domain. The intrinsic susceptibility of a multidomain grain is therefore related to the intrinsic susceptibilities of domains having magnetocrystalline anisotropy and to the shapes of these domains. Elongations or alinements of domains can be detected in weak applied fields, provided that the grain has not been subjected to strong demagnetizing fields (Stacey, 1961).

\section{APPARENT SUSCEPTIBILITY OF A SPHERICAL GRAIN}

When a magnetic body of finite dimensions, such as a magnetic mineral grain, is exposed to an external magnetic field, a demagnetizing field is established within the grain opposite in direction to the external field. The applied field within the grain, fundamental to the definition of intrinsic magnetic susceptibility given in equation 1, consists of the vector sum of external and demagnetizing fields. Because the external field is much easier to measure than the internal field, it is convenient to determine experimentally the apparent magnetic susceptibility, $\widetilde{\mathbf{K}}_{\boldsymbol{A}}$, given by relation 8 ,

$$
\overrightarrow{\boldsymbol{J}}=\tilde{\mathbf{K}}_{\boldsymbol{A}} \cdot \overrightarrow{\boldsymbol{H}}_{\text {ext }}
$$

The apparent magnetic susceptibility satisfies all the second-rank tensoral properties of intrinsic magnetic susceptibility and is generally anisotropic.

The origin of the internal demagnetizing field of a mineral grain may be analytically demonstrated ${ }^{4}$ by using a method of potential field analysis credited to Poisson (Maxwell, 1892, art. 437-438). This method relates the magnetic potential, $\Omega$, of a body having uniform magnetization to the gravitational potential, V,

- The internal demagnetizing field is classically pictured (Williams, 1931; Bitter, 1937) as the return lines of magnetic force associated with equatorial regions of magnetic dipoles within a body. Although these lines of force vectorially add to the external field in polar regions of each dipole, they subtract from the field in equatorial regions and are therefore equivalent to a demagnetizing field. 
of that body, modified by replacing a constant-density term by a magnetization term. Poisson's relation may be written

$$
\Omega=-\frac{d \mathrm{~V}}{d \xi},
$$

where $\xi$ is the direction of magnetization, the gravitational contant taken as unity. The internal demagnetizing field, $\boldsymbol{H}_{\mathrm{d}}$, is obtained by taking the negative gradient of the magnetic potential,

$$
\overrightarrow{\boldsymbol{H}}_{\mathrm{d}}=-\nabla \boldsymbol{\Omega} \text {. }
$$

The demagnetizing field component in the $\xi$ direction is therefore

$$
\left(H_{\mathrm{d}}\right)_{\xi}=\frac{d^{2} \mathrm{~V}}{d \xi^{2}}
$$

The requirement of uniform magnetization for application of Poisson's relation is not in itself also a requirement that the internal demagnetizing field be uniform. However, if the external field is made uniform by experimental design, Poisson's relation will hold only if the demagnetizing field is also uniform. Further, if the demagnetizing field is uniform, the left-hand side of equation 9 is constant, and integration of equation 10 indicates that the modified gravitational potential, V, must be a quadratic function of coordinates within the body. As indicated by Maxwell (1892, art. 437), the only cases known for which this potential is a quadratic function of coordinates of a finite body are those in which the body is bounded by a complete surface of the second degree, namely, an ellipsoid. Therefore, a finite body, such as a mineral grain, exposed to a uniform external field will have a uniform magnetization and internal demagnetizing field only if it has the shape of a triaxial ellipsoid, spheroid (ellipsoid of revolution), or sphere.

As an illustration of applying Poisson's relation for determining the internal demagnetizing field of a body, we write the modified gravitational potential for a spherical mineral grain magnetized in the $+x$ direction as

$$
\mathrm{V}=2 / 3 \pi J_{x}\left(3 a^{2}-\mathrm{X}^{2}-\mathrm{Y}^{2}-\mathrm{Z}^{2}\right),
$$

where $J_{x}$ is the magnetization, $a$ is the radius of the sphere, and $\mathrm{X}, \mathrm{Y}$, and $\mathrm{Z}$ are cartesian components of a point inside the sphere. Using Poisson's relation (equation 9), we have for the magnetic potential

$$
\Omega=-\frac{d \mathrm{~V}}{d \mathrm{X}}=4 / 3 \pi J_{x} \mathrm{X}
$$


The internal demagnetizing field, obtained by using equation 10 is

$$
\left(H_{\mathrm{d}}\right)_{x}=\frac{d^{2} \mathrm{~V}}{d \mathrm{X}^{2}}=-4 / 3 \pi J_{x} .
$$

If the spherical grain were magnetized along either the $+y$ or $+z$ direction instead of along the $+x$ direction, similar equations result, namely

and

$$
\left(H_{\mathrm{d}}\right)_{v}=-4 / 3 \pi J_{y}
$$

$$
\left(H_{\mathrm{d}}\right)_{z}=-4 / 3 \pi J_{z} .
$$

For the special case of a sphere, we may write

$$
H_{\mathrm{d}}=-4 / 3 \pi J
$$

valid for any direction of magnetization. If we define the demagnetizing factor, $\mathrm{N}$, of the spherical body by the relation

$$
H_{\mathrm{d}} \stackrel{\Delta}{=} \mathrm{N} J \text {, }
$$

we see by direct substitution that

$$
\mathrm{N}=\frac{4 \pi}{3}
$$

As alternatives to the method of Poisson used above, methods based on vector analysis (Stoner, 1945) and the application of Gauss' Theorum (see, for example, Chikazumi, 1964) may be used to derive the demagnetizing field and associated demagnetizing factor for a uniformly magnetized body.

If, in experimental work, the apparent magnetic susceptibility is measured, the intrinsic magnetic susceptibility must be obtained by computation. Considering a spherical grain having an isotropic intrinsic susceptibility, we may write for the applied field inside of the grain

$$
H=H_{\text {ext }}-\mathrm{N} J,
$$

and for the induced magnetization at that internal point

$$
\begin{aligned}
J & =\mathrm{K} H \\
& =\mathrm{K}\left(H_{\mathrm{ext}}-\mathrm{N} J\right) .
\end{aligned}
$$

Because, from equation 3,

$$
J=\left(\frac{\mathrm{K}}{1+\mathrm{NK}}\right) H_{\text {ext }},
$$

and by direct substitution, we have 


$$
\mathrm{K}_{\mathrm{A}}=\frac{\mathrm{K}}{1+\mathrm{NK}}
$$

or, alternatively,

$$
\mathrm{K}=\frac{\mathrm{K}_{\mathbf{A}}}{1-\mathrm{NK}_{\mathrm{A}}}
$$

provided that

$$
\mathrm{K}_{\mathbf{\Delta}}<\frac{1}{\mathrm{~N}}, \mathrm{~K}>0, \mathrm{~N}>0,
$$

and that $\mathrm{K}$ is finite, conditions valid for magnetic minerals.

If we substitute the numerical value of $\mathrm{N}$ for the sphere into 12,13 , and 14 , we have

$$
\mathrm{K}_{\mathrm{A}}=\frac{3 \mathrm{~K}}{3+4 \pi \mathrm{K}}
$$

and

$$
\mathrm{K}=\frac{3 \mathrm{~K}_{\mathbf{A}}}{3-4 \pi \mathrm{K}_{\mathrm{A}}}
$$

provided that

$$
\mathrm{K}_{\mathrm{A}}<\frac{3}{4 \pi}
$$

Relation 16 indicates that the maximum value of apparent susceptibility for a uniformly magnetized spherical grain is $2.39 \times$ $10^{-1} \mathrm{emu} / \mathrm{cm}^{3}$, no matter how high the intrinsic susceptibility value. Considering that naturally occurring titaniferous magnetite has an intrinsic susceptibility which ranges from $5 \times 10^{-2}$ to $5 \times 10^{-1} \mathrm{emu} / \mathrm{cm}^{3}$ (Nagata and Akimoto, 1961, p. 99), we see from equation 15 that spherical grains of this magnetite would have an apparent susceptibility in the range of $4.13 \times 10^{-2}$ to $1.62 \times 10^{-1} \mathrm{emu} / \mathrm{cm}^{3}$. For a representative intrinsic susceptibility of $10^{-1} \mathrm{emu} / \mathrm{cm}^{3}$, the corresponding apparent susceptibility for a spherical grain is $7.05 \times 10^{-2} \mathrm{emu} / \mathrm{cm}^{3}$. It is of interest that the apparent and intrinsic susceptibilities of a uniformly magnetized spherical grain agree to within 1 percent if the intrinsic susceptibility is less than $2.41 \times 10^{-3} \mathrm{emu} / \mathrm{cm}^{3}$; they agree to within 10 percent if the intrinsic susceptibility is less than $2.65 \times$ $10^{-2} \mathrm{emu} / \mathrm{cm}^{3}$.

It may be noted that relations 12 and 13, written for a spherical grain having isotropic intrinsic susceptibility, ${ }^{5}$ are also valid along

\footnotetext{
5 For example, magnetite has an isotropic intrinsic magnetic susceptibility in a sufficiently weak applied field.
} 
each of three orthogonal axes in a spherical grain having anisotropic intrinsic susceptibility. In these expressions, $\mathrm{K}$ and $\mathrm{K}_{\mathbf{A}}$ are replaced by their corresponding values along a particular principal axis of the anisotropic grain. The anisotropy of apparent susceptibility of such an anisotropic spherical grain is associated entirely with its anisotropy of intrinsic susceptibility and not at all with its external shape.

\section{SHAPE ANISOTROPY OF AN ELLIPSOIDAL GRAIN}

Although any apparent susceptibility anisotropy possessed by a spherical grain must be associated entirely with its intrinsic anisotropy, the apparent anisotropy of an ellipsoidal grain is associated largely with its shape. For the ideal case of an intrinsically isotropic ellipsoidal grain, apparent anisotropy is associated entirely with grain shape. The relative contributions of intrinsic anisotropy and grain shape to apparent anisotropy depend upon the crystal symmetry, composition, and shape of the mineral grain. For example, experimental work of Uyeda, Fuller, Belshé, and Girdler (1963) indicates that apparent anisotropy of most naturally occurring grains of high-symmetry minerals, such as magnetite, is associated more with grain shape than intrinsic anisotropy (magnetocrystalline anisotropy). Conversely, apparent anisotropy of most naturally occurring lower symmetry minerals, such as hematite, is associated more with intrinsic anisotropy than with grain shape.

It is convenient to discuss the concept of shape anisotropy by considering an ellipsoidal grain having isotropic intrinsic susceptibility. Just as intrinsic anisotropy derives from a tensoral relationship between two vectors in relation 3 , shape anisotropy derives from a tensoral relationship between the demagnetizing field vector and the induced magnetization vector. If we extend the relation 11 to include cartesian coordinates of a vector, we may rewrite the expression by the three component equations

$$
\begin{aligned}
& \left(H_{\mathrm{d}}\right)_{\mathrm{x}}=-\left(N_{x x} J_{x}+N_{x y} J_{y}+N_{x z} J_{z}\right), \\
& \left(H_{\mathrm{d}}\right)_{y}=-\left(N_{y x} J_{x}+N_{y y} J_{y}+N_{y z} J_{z}\right),
\end{aligned}
$$

and

$$
\left(H_{\mathrm{d}}\right)_{z}=-\left(N_{z x} J_{x}+N_{z y} J_{y}+N_{z z} J_{z}\right),
$$

where the demagnetizing factor terms are components of a symmetrical second-rank tensor.

Because of the complexity of the analytical expression for the potential (gravitational or magnetic) of a triaxial ellipsoid, the 
components of demagnetization factor for a triaxial-ellipsoidal grain cannot be written in a simple form. Instead, these components are ordinarily expressed in terms of elliptic integrals (Stoner, 1945), and numerical values of the components may be tabulated. If two principal axes of the ellipsoid are equal, as in a spheroid or ellipsoid of revolution, the analytical expression for potential is simpler, and demagnetization factors are more easily tabulated. For example, the demagnetizing factors for prolate and oblate spheroids, first derived by Maxwell (1892) and later evaluated by Stoner (1945), may be expressed by equations given in simple form by Nagata and Kobayashi $(1961$, p. 70$)$ as Prolate spheroid

$$
\begin{aligned}
& N_{x}=4 \pi\left(\frac{1-e^{2}}{e^{2}}\right)\left[\frac{1}{2 e} \ln \left(\frac{1+e}{1-e}\right)-1\right], \\
& N_{\nu}=N_{z}=2 \pi\left[\frac{1}{e^{2}}-\left(\frac{1-e^{2}}{2 e^{2}}\right) \ln \left(\frac{1+e}{1-e}\right)\right],
\end{aligned}
$$

where

$$
e \triangleq \frac{\sqrt{a^{2}-b^{2}}}{a}, a>b=c, \text { and }
$$

Oblate spheroid

$$
\begin{aligned}
& N_{x}=4 \pi\left[\frac{1}{e^{2}}-\frac{\sqrt{1-e^{2}}}{e^{3}} \sin ^{-1} e\right], \\
& N_{\nu}=N_{z}=2 \pi\left[\left(\frac{\sqrt{1-e^{2}}}{e^{3}}\right) \sin ^{-1} e-\left(\frac{1-e^{2}}{e^{3}}\right)\right],
\end{aligned}
$$

where

$$
e=\frac{\sqrt{b^{2}-a^{2}}}{b}, b=c>a .
$$

In these expressions, $a$ is the symmetry axis, or axis of revolution of the grain, and $b$ and $c$ are principal axes of equal length in the principal plane of symmetry. The coordinate $x$ coincides with $a, y$ with $b$, and $z$ with $c$.

From expressions 17 through 20, graphs of anisotropy factor, $P$, defined by

$$
P=\frac{1+\mathrm{K} N_{\nu}}{1+\mathrm{K} N_{x}}
$$

have been plotted as a function of the dimension ratio, $m$, of the spheroidal grain, defined by 


$$
m=\frac{b}{a},
$$

where $\mathrm{K}$ is the intrinsic susceptibility of the grain, which is assumed to be isotropic (Uyeda, Fuller, Belshé, and Girdler, 1963, p. 280-281). As an illustration of the use of such graphs, it may be quickly determined that the ratio of principal apparent susceptibilities of a prolate spheroidal grain having a major axis twice as long as its minor axis and an intrinsic susceptibility of $5 \times 10^{-2} \mathrm{emu} / \mathrm{cm}^{3}$ is 1.14 to 1 . Computation of this result would be an extremely tedious task without the use of a programmable calculator or a digital computer.

Expressions 17 through 20 may also be used to deduce the maximum and minimum values possible for an ellipsoid, and, therefore, for a uniformly magnetized grain. For a slender prolate spheroidal grain having the shape of a needle,

$$
N_{x}=0 \text { and } N_{y}=N_{z}=2 \pi \text {. }
$$

For a flat oblate spheroidal grain having the shape of a disc,

$$
N_{x}=4 \pi \text { and } N_{y}=N_{z}=0 .
$$

Thus, we see that the limits of values of $N$ for a finite body are

$$
0 \leq N \leq 4 \pi \text {. }
$$

These limits for values of $N$, in turn, set limits on maximum and minimum values of apparent susceptibility in an ellipsoidal grain as previously discussed.

Although the demagnetizing factor is strictly defined only for uniform magnetization, and, therefore, for ellipsoidal bodies, good approximations to $\tilde{\mathbf{N}}$ may be obtained for bodies having nonellipsoidal shapes (Bozorth and Chapin, 1942; Jahren, 1963; Olsen, 1966; Sharma, 1968). Thus, equations 17 through 20 can be applied to a wide variety of shapes of finite bodies, depending upon required precision of the measurement or calculation. For example, susceptibility anisotropy investigations of Uyeda, Fuller, Belshé, and Girdler (1963) have been successfully carried out using cylindrical specimens of rocks and minerals, approximated to spheroidal shapes having orthogonal axes of the same dimensions (lengths) as axes of the cylinders.

\section{COMBINATIONS OF INTRINSIC SUSCEPTIBILITY AND SHAPE ANISOTROPIES}

The apparent magnetic susceptibility tensor, $\tilde{\mathbf{K}}_{A}$, of a uniformly magnetized body measured in a weak magnetic field is a function of both the intrinsic susceptibility tensor, $\tilde{\mathbf{K}}$, of the magnetic ma- 
terial, and the demagnetization factor tensor, $\tilde{\mathbf{N}}$, associated with the shape of the body. The fundamental relationship among these three tensor quantities, one a dependent variable and the others independent variables, may be developed in the following way.

If $\overrightarrow{\boldsymbol{J}}, \tilde{\mathbf{K}}_{\boldsymbol{A}}, \overrightarrow{\boldsymbol{H}}_{\text {ext }}, \tilde{\mathbf{K}}, \overrightarrow{\boldsymbol{H}}_{\text {eff }}$, and $\tilde{\mathbf{N}}$ are the induced magnetization vector, apparent susceptibility tensor, external field vector, intrinsic susceptibility tensor, effective field vector, and demagnetization factor tensor, respectively, of a uniformly magnetized body, we may write, using dyadic notation of Gibbs (Wilson, 1909)

$$
\begin{gathered}
\overrightarrow{\boldsymbol{J}}=\tilde{\mathbf{K}}_{\boldsymbol{A}} \cdot \overrightarrow{\boldsymbol{H}}_{\text {ext }} \\
=\tilde{\mathbf{K}} \cdot \overrightarrow{\boldsymbol{H}}_{\text {eff }}, \\
\overrightarrow{\boldsymbol{H}}_{\text {eff }}=\overrightarrow{\boldsymbol{H}}_{\text {ext }}-\overrightarrow{\boldsymbol{N}} \cdot \overrightarrow{\boldsymbol{J}}, \\
\overrightarrow{\boldsymbol{J}}=\tilde{\mathbf{K}} \cdot\left(\overrightarrow{\boldsymbol{H}}_{\text {ext }}-\tilde{\mathbf{N}} \cdot \overrightarrow{\boldsymbol{J}}\right) \\
=\tilde{\mathbf{K}} \cdot \overrightarrow{\boldsymbol{H}}_{\text {ext }}-(\tilde{\mathbf{K}} \cdot \tilde{\mathbf{N}}) \cdot \overrightarrow{\boldsymbol{J}} .
\end{gathered}
$$

If $\tilde{\mathbf{I}}$ is the idemfactor of Gibbs (Wilson, 1909, p. 288),

$$
\tilde{\mathbf{I}} \cdot \vec{J}=\vec{J}
$$

and

$$
[\tilde{\mathbf{I}}+(\tilde{\mathbf{K}} \cdot \tilde{\mathbf{N}})] \cdot \overrightarrow{\boldsymbol{J}}=\tilde{\mathbf{K}} \cdot \overrightarrow{\boldsymbol{H}}_{\text {ext }},
$$

then

$$
\overrightarrow{\boldsymbol{J}}=\left\{[\tilde{\mathbf{I}}+(\tilde{\mathbf{K}} \cdot \tilde{\mathbf{N}})]^{-1} \cdot \tilde{\mathbf{K}}\right\} \cdot \overrightarrow{\boldsymbol{H}}_{\text {ext }} .
$$

The apparent susceptibility is therefore

$$
\tilde{\mathbf{K}}_{A}=[\tilde{\mathbf{I}}+(\tilde{\mathbf{K}} \cdot \tilde{\mathbf{N}})]^{-1} \cdot \tilde{\mathbf{K}} \text {. }
$$

Tensor components of $\tilde{\mathbf{K}}_{A}$, referred to a coordinate system $\left(x_{1}\right.$, $x_{2}, x_{3}$ ) fixed to the uniformly magnetized body, are

$$
\begin{aligned}
& \left(K_{A}\right)_{11}=\left[K_{11}(\mathrm{EI}-\mathrm{FH})+K_{21}(\mathrm{CH}-\mathrm{BI})+K_{s 1}(\mathrm{BF}-\mathrm{CE})\right] / \mathrm{L}, \\
& \left(K_{A}\right)_{12}=\left[K_{12}(\mathrm{EI}-\mathrm{FH})+K_{22}(\mathrm{CH}-\mathrm{BI})+K_{s 2}(\mathrm{BF}-\mathrm{CE})\right] / \mathrm{L}, \\
& \left(K_{A}\right)_{1 s}=\left[K_{1 s}(\mathrm{EI}-\mathrm{FH})+K_{2 s}(\mathrm{CH}-\mathrm{BI})+K_{s s}(\mathrm{BF}-\mathrm{CE})\right] / \mathrm{L}, \\
& \left(K_{A}\right)_{21}=\left[K_{11}(\mathrm{FG}-\mathrm{DI})+K_{21}(\mathrm{AI}-\mathrm{CG})+K_{s 1}(\mathrm{CD}-\mathrm{AF})\right] / \mathrm{L}, \\
& \left(K_{A}\right)_{22}=\left[K_{12}(\mathrm{FG}-\mathrm{DI})+K_{22}(\mathrm{AI}-\mathrm{CG})+K_{s 2}(\mathrm{CD}-\mathrm{AF})\right] / \mathrm{L}, \\
& \left(K_{A}\right)_{2 s}=\left[K_{1 s}(\mathrm{FG}-\mathrm{DI})+K_{2 s}(\mathrm{AI}-\mathrm{CG})+K_{s s}(\mathrm{CD}-\mathrm{AF})\right] / \mathrm{L}, \\
& \left(K_{A}\right)_{s 1}=\left[K_{11}(\mathrm{DH}-\mathrm{EG})+K_{21}(\mathrm{BG}-\mathrm{AH})+K_{s 1}(\mathrm{AE}-\mathrm{BD}] / \mathrm{L},\right. \\
& \left(K_{A}\right)_{s 2}=\left[K_{12}(\mathrm{DH}-\mathrm{EG})+K_{22}(\mathrm{BG}-\mathrm{AH})+K_{s 2}(\mathrm{AE}-\mathrm{BD}] / \mathrm{L},\right. \\
& \left(K_{A}\right)_{s s}=\left[K_{1 s}(\mathrm{DH}-\mathrm{EG})+K_{2 s}(\mathrm{BG}-\mathrm{AH})+K_{s s}(\mathrm{AE}-\mathrm{BD}] / \mathrm{L},\right.
\end{aligned}
$$


where

$$
\begin{aligned}
\mathrm{L}=\mathrm{A}(\mathrm{EI}-\mathrm{FH})+\mathrm{B}(\mathrm{FG}-\mathrm{DI})+\mathrm{C}(\mathrm{DH}-\mathrm{EG}), \\
\mathrm{A}=1+K_{11} N_{11}+K_{12} N_{21}+K_{1 s} N_{s 1}, \\
\mathrm{~B}=K_{11} N_{12}+K_{12} N_{22}+K_{1 s} N_{s 2}, \\
\mathrm{C}=K_{11} N_{1 s}+K_{12} N_{2 s}+K_{1 s} N_{s s} \\
\mathrm{D}=K_{21} N_{11}+K_{22} N_{21}+K_{2 s} N_{s 1}, \\
\mathrm{E}=1+K_{21} N_{12}+K_{22} N_{22}+K_{2 s} N_{s 2}, \\
\mathrm{~F}=K_{21} N_{1 s}+K_{22} N_{2 s}+K_{2 s} N_{s s}, \\
\mathrm{G}=K_{s 1} N_{11}+K_{s 2} N_{21}+K_{s s} N_{s 1}, \\
\mathrm{H}=K_{s 1} N_{12}+K_{s 2} N_{22}+K_{s s} N_{s 2}, \\
\mathrm{I}=1+K_{s 1} N_{1 s}+K_{s 2} N_{2 s}+K_{s s} N_{s s} .
\end{aligned}
$$

Using the symmetric properties

$$
K_{12}=K_{21}, K_{1 s}=K_{s 1}, K_{2 s}=K_{s 2},
$$

and

$$
N_{12}=N_{21}, N_{13}=N_{31}, N_{2 s}=N_{32} \text {, }
$$

we may, by direct substitution, obtain the expected result

$$
\left(K_{A}\right)_{12}=\left(K_{4}\right)_{21},\left(K_{A}\right)_{1 s}=\left(K_{4}\right)_{s 1},\left(K_{4}\right)_{2 s}=\left(K_{A}\right)_{s 2} \text {. }
$$

If $\tilde{\mathbf{K}}$ is isotropic and $\tilde{\mathbf{N}}$ is anisotropic, relation 21 reduces to

$$
\begin{aligned}
& \left(\mathrm{K}_{\mathrm{A}}\right)_{1}=\frac{\mathrm{K}}{1+\mathrm{KN}_{1}}, \\
& \left(\mathrm{~K}_{\mathrm{A}}\right)_{2}=\frac{\mathrm{K}}{1+\mathrm{KN}_{2}}, \\
& \left(\mathrm{~K}_{\mathrm{A}}\right)_{3}=\frac{\mathrm{K}}{1+\mathrm{KN}_{3}},
\end{aligned}
$$

where the subscripts $1,2,3$, refer to the three orthogonal principal axes of $\tilde{\mathbf{N}}$.

If $\tilde{\mathbf{N}}$ is isotropic and $\tilde{\mathbf{K}}$ is anisotropic, relation 21 reduces to

$$
\begin{aligned}
& \left(\mathrm{K}_{\mathrm{A}}\right)_{1}=\frac{\mathrm{K}_{1}}{1+\mathrm{NK}_{1}}, \\
& \left(\mathrm{~K}_{\mathrm{A}}\right)_{2}=\frac{\mathrm{K}_{2}}{1+\mathrm{NK}_{2}}, \\
& \left(\mathrm{~K}_{\mathrm{A}}\right)_{3}=\frac{\mathrm{K}_{3}}{1+\mathrm{NK}_{3}},
\end{aligned}
$$

when referred to the principal axes of $\tilde{\mathbf{K}}$.

If both $\tilde{\mathbf{K}}$ and $\tilde{\mathbf{N}}$ are isotropic, relation 21 reduces to the single expression 


$$
\mathrm{K}_{\mathrm{A}}=\frac{\mathrm{K}}{1+\mathrm{NK}}
$$

Although a combination of anisotropic $\tilde{\mathbf{K}}$ and anisotropic $\tilde{\mathbf{N}}$ generally gives rise to an anisotropic $\widetilde{\mathbf{K}}_{A}$, this combination may produce an isotropic $\tilde{\mathbf{K}}_{\boldsymbol{A}}$ under fortuitous circumstances. If the principal axes of $\widetilde{\mathbf{K}}$ happen to coincide with the principal axes of $\tilde{\mathbf{N}}$, and if the relative magnitudes of principal components of $\tilde{\mathbf{K}}$ and $\tilde{\mathbf{N}}$ are in the right proportion, an isotropic $\tilde{\mathbf{K}}_{\boldsymbol{A}}$ will result. For example, if $\mathrm{K}_{1}=1 \mathrm{emu} / \mathrm{cm}^{3}, \mathrm{~K}_{2}=2 \mathrm{emu} / \mathrm{cm}^{3}, \mathrm{~K}_{3}=3 \mathrm{emu} / \mathrm{cm}^{3}, \mathrm{~N}_{1}=$ $1, \mathrm{~N}_{2}=3 / 2$, and $\mathrm{N}_{3}=5 / 3$, an isotropic $\mathrm{K}_{\mathrm{A}}=1 / 2 \mathrm{emu} / \mathrm{cm}^{3}$ results. In naturally occurring materials, such combinations of alined $\tilde{\mathbf{K}}$ and $\tilde{\mathbf{N}}$ principal axes and relative magnitudes of principal components are not likely to occur.

The tensors $\tilde{\mathbf{K}}$ and $\tilde{\mathbf{N}}$ can combine in two ways to produce an isotropic $\widetilde{\mathbf{K}}_{A}$ and in three ways to produce an anisotropic $\widetilde{\mathbf{K}}_{A}$. These tensor combinations are summarized in figure 4 . These combinations may be analyzed in terms of magnetic bodies of various scales, such as a single magnetic domain, combinations of magnetic domains composing mineral grains, combinations of mineral grains composing magnetic rock units (naturally occurring units or specimens cut from such units), and combinations of rock units composing magnetic rock formations. As an illustration of possible tensor combinations, figure 4 shows that $13 \tilde{\mathbf{K}}$ and $\tilde{\mathbf{N}}$ combinations of single magnetic domains may give rise to an isotropic $\tilde{\mathbf{K}}_{A}$ of a magnetic rock formation, depending upon how these domains are distributed in multidomain mineral grains and magnetic rock units. Twenty-one $\tilde{\mathbf{K}}$ and $\tilde{\mathbf{N}}$ combinations of single domains may give rise to an anisotropic $\tilde{\mathbf{K}}_{A}$ of a magnetic rock formation. For a specimen cut from a magnetic rock unit, five $\tilde{\mathbf{K}}$ and $\tilde{\mathbf{N}}$ combinations of single domains can produce an isotropic $\tilde{\mathbf{K}}_{A}$, and eight combinations can produce an anisotropic $\tilde{\mathbf{K}}_{A}$. As noted previously, laboratory investigations of Uyeda, Fuller, Belshé, and Girdler (1963) suggest that the effects of anisotropic $\tilde{\mathbf{K}}$ and $\tilde{\mathbf{N}}$ in rocks and minerals are often separable. In general, the $\tilde{\mathbf{N}}$ term predominates in rocks composed of cubic titanomagnetites. The $\tilde{\mathbf{K}}$ term predominates in rocks composed of lower symmetry magnetic minerals, such as hematites, ilmenites, and pyrrhotites. In rocks for which $\tilde{\mathbf{K}}$ is very small in magnitude, anisotropy of $\tilde{\mathbf{K}}_{A}$ will be small, regardless of the magnitude of $\tilde{\mathbf{N}}$.

\section{INTRINSIC SUSCEPTIBILITY OF A ROCK BODY}

The intrinsic susceptibility of a body of magnetic rock, whether a naturally occurring rock unit or a specimen cut from such a 


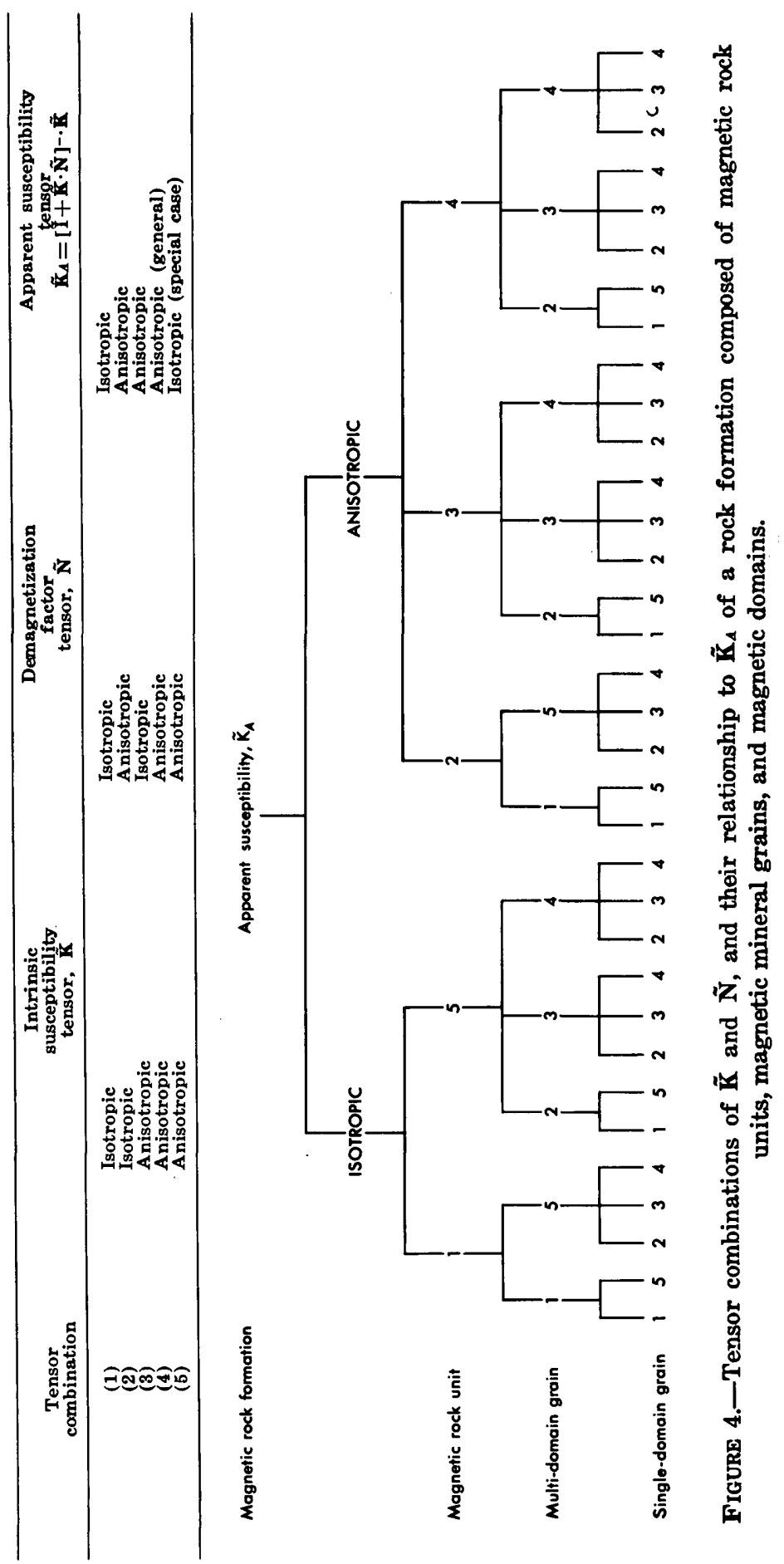


unit, depends upon the intrinsic susceptibilities and shapes of magnetic mineral grains and magnetic domains composing such grains within the rock unit. The relative contributions of intrinsic susceptibility and shape anisotropy of magnetic constituents of a rock can be determined only by a thorough microscopic analysis of grain and domain shapes, magnetizations, and compositions using techniques such as those described by Stoner (1934), Bates (1961), Chikazumi (1964), and Zijlstra (1967). In practice, however, measurements of intrinsic susceptibility anisotropy of rocks are often related to characteristics of magnetic constituents by using an assumed model. For example, a model of Nagata and Uyeda (1961) may be used to relate measured anisotropic $\tilde{\mathbf{K}}$ of rocks containing intrinsically isotropic titanomagnetite grains to the shapes of these grains.

Theoretical models of Stacey, Joplin, and Lindsay (1960) relate intrinsic susceptibilities of magnetic mineral grains to demagnetizing factors associated with shapes of the grains. In studies of igneous rocks, they suggest that a good approximation to average grain shape is the dimension ratio $1.65: 1.45: 1$ of a triaxial ellipsoid. Where this double ratio for a triaxial ellipsoid was converted to a typical single ratio of 1.5:1 for an oblate spheroid, the authors computed that a randomly oriented distribution of such spheroidal particles in a rock would result in a mean isotropic demagnetizing factor of 3.91. This mean factor is within 7 percent of the equivalent demagnetizing factor of $\frac{4 \pi}{3}$ for a similar distribution of spherical particles. Stacey (1961) further pointed out that an assembly of grains containing domains having random orientations has a mean isotropic intrinsic susceptibility of

$$
\mathrm{K}=1 / 3\left(\mathrm{~K}_{\mathrm{par}}+2 \mathrm{~K}_{\mathrm{per}}\right) \text {, }
$$

where $\mathrm{K}$ is the intrinsic susceptibility, $\mathrm{K}_{\mathrm{par}}$ is the weak-field susceptibility of the magnetic material measured parallel to its domain directions, and $\mathrm{K}_{\text {per }}$ is the susceptibility measured perpendicular to its domain directions.

In the model of Nagata and Uyeda (1961, p. 130), each magnetic mineral grain is identical, and each is assumed to be surrounded by a small hollow cavity in the continuous phase of the rock. If $p, K, N, L$, and $M$ are, respectively, the volume percentage of magnetic mineral grains within the rock, the isotropic intrinsic susceptibility of a single mineral grain, the demagnetizing factor of the grain, the demagnetizing factor of the rock specimen, and 
the demagnetizing factor of the cavity, then the apparent susceptibility, $\widetilde{\mathbf{K}}_{A}$, of the rock specimen is

$$
\mathrm{K}_{\mathrm{A}}=\frac{\mathrm{pK}}{1+[\mathrm{N}+(\mathrm{L}-\mathrm{M}) \mathrm{p}] \mathrm{K}} \text {. }
$$

If the shape of the cavity is assumed to be identical with the shape of the specimen,

and

$$
\mathrm{L}=\mathbf{M} \text {, }
$$

$$
\mathrm{K}_{\mathrm{A}}=\frac{\mathrm{pK}}{1+\mathrm{NK}} \text {. }
$$

Measurements of $\mathrm{K}_{\mathrm{A}}$ and estimates of $\mathrm{p}$ for rocks can be used to compute $\mathrm{N}$, which is the mean demagnetizing factor of the rocks. Computed $\mathrm{N}$ values are ordinarily found to be less than the $\mathrm{N}$ value for a distribution of spherical particles. This fact suggests that the particles are elongate, a conclusion also reached in the investigations by Stacey, Joplin, and Lindsay (1960).

As noted by Graham $(1954,1967)$ and Balsley and Buddington (1960) in studies of rock susceptibilities, large numbers of irregularly shaped mineral grains within a rock can be expected to be dispersed into relatively simple population distributions. For example, grains that have flat or platy dimensions tend to aline parallel to planar features of rocks, such as bedding in sedimentary or volcanic rocks and foliation in metamorphic rocks. Grains that are elongate tend to aline parallel to linear structures of rocks, such as phenocryst alinements in volcanic flows and lineations in metamorphic rocks.

\section{MINIMIZING ANISOTROPY CAUSED BY ROCK SPECIMEN SHAPE}

The only requirement for eliminating the shape effect of rock specimens used in susceptibility anisotropy measurements is to know the geometries of the specimens to the precision of the measurement; however, it is convenient to use equidimensional specimens in practice to avoid excessive computation. In many rock-magnetic laboratories, specimens having the shape of a sphere, cube, cylinder, or volume formed by the orthogonal intersection of two or three cylinders are used. Calculations of Sharma $(1968$, p. 132) show that demagnetizing factors associated with orthogonal axes of a cube are identical with the demagnetizating factor of a sphere. As noted by Jahren (1963); calculations by Werner (1945) show that the demagnetizing factors associated with orthogonal axes of a cylinder are equal to that of a sphere, provided the length-to-diameter ratio of the cylinder is 0.89 . A 
more recent computation by Noltimier (1971) indicates that the optimal length-to-diameter ratio for a uniformly magnetized cylinder is 0.865 , if shape anisotropy is to be minimized. Experiments of Porath, Stacey, and Cheam (1966) on cylinders of iron and nickel indicate that the optimal length-to-diameter ratio in weak magnetic fields is 0.85 and in strong magnetic fields, 0.88 . It may be noted that the length-to-diameter ratio of rock cores used by Doell and Cox (1965) to minimize nondipolar components of remanent magnetization was about 0.92 . To one significant figure, the optimal length-to-diameter ratio may be taken to be 0.9 .

\section{APPARENT SUSCEPTIBILITY ANISOTROPY OF A ROCK FORMATION}

The largest entity to which the concept of anisotropic susceptibility is ordinarily applied is the magnetic rock formation, consisting of a number of magnetic rock bodies or units. If the magnitude of intrinsic susceptibility of the formation is very large, say, greater than $10^{-2} \mathrm{emu} / \mathrm{cm}^{3}$, the susceptibility anisotropy will affect computation of magnetic anomalies associated with a model of the formation. In analyzing magnetic anomalies associated with strongly magnetized rocks, therefore, it is helpful either, to compute demagnetizing effects directly (Sharma, 1966) during computation of the magnetic field or to correct for shape anisotropy after uncompensated computations have been made. The gross geometric relationships among magnetic rock formation, rock unit, specimen, mineral grain, and magnetic domain are shown in figure 5 .

\section{ANALYSIS OF SUSCEPTIBILITY ANISOTROPY OF A ROCK SPECIMEN}

\section{TENSOR COMPONENTS OF LINEAR SUSCEPTIBILITIES}

The quantity of direct interest in most studies of magnetic susceptibility anisotropy is the intrinsic susceptibility anisotropy of a rock. This quantity may, with certain assumptions, be related to mean shape anisotropies of magnetite grains within the rock, or magnetic domains composing individual grains within the rock. As this property of a rock is ordinarily determined by using finite specimens of the rock, the quantity actually measured is apparent magnetic susceptibility anisotropy. The apparent susceptibility anisotropy can be most easily related to desired intrinsic susceptibility anisotropy if the shape of the rock specimen 


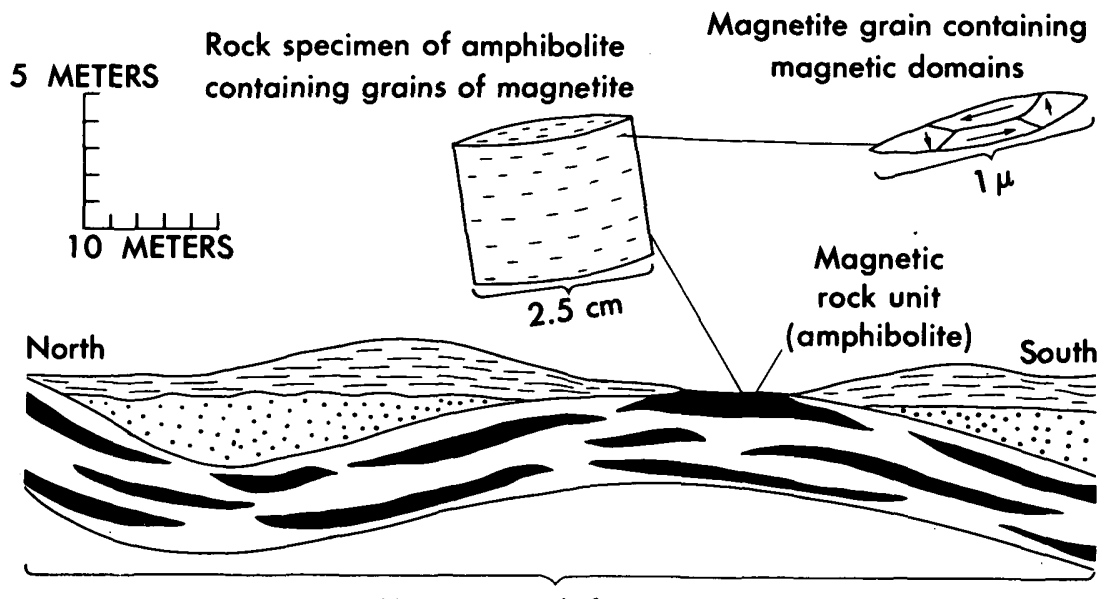

Magnetic rock formation

(Layer of amphibolite-quartzofeldspathic gneiss)

FIgURE 5.-Idealized sketch of material entities possessing magnetic susceptibility anisotropy, including a magnetic rock formation, magnetic rock units, magnetic rock specimen, magnetic mineral grains, and magnetic domains.

is equidimensional. Specimens having the shape of a sphere, cube, cylinder having length-to-diameter ratio of about 0.9 , or volume formed by the orthogonal intersection of two or three identical cylinders are ideal for use in laboratory work. The uniformly magnetized equidimensional rock specimen forms the practical basis here for quantitatively analyzing magnetic susceptibility anisotropy.

The linear relationship between the induced magnetization vector $\vec{J}$ of a uniformly magnetized equidimensional rock specimen and an external magnetic field vector, $\overrightarrow{\boldsymbol{H}}$, applied throughout the volume of the rock specimen is expressed by relation 8 .

$$
\overrightarrow{\boldsymbol{J}}=\tilde{\mathbf{K}} \cdot \overrightarrow{\boldsymbol{H}}
$$

where $\tilde{\mathbf{K}}$ is the apparent magnetic susceptibility of the rock specimen. As noted previously, $\widetilde{\mathbf{K}}$ is a second-rank tensor ${ }^{6}$ having, in general, nine components which may be expressed in dyadic, matrix, or subscript form. The dyadic form has been used in a previous section to describe apparent magnetic susceptibility, and we shall have occasion to use the matrix and subscript forms to describe this quantity in various contexts. Discussions of these

6 The second-rank tensor property of apparent magnetic susceptibility has been previously noted by Nagata and Uyeda (1961) and Coe (1966). 
forms of tensor expression may be found in Wilson (1909), Wills (1931), Nye (1960), Symon (1960), Tropper (1962), Post (1962), Sokolnikoff (1964), Hollingsworth (1967), Borisenko and Tarapov (1968), and Billings (1969). For a specimen characterized by anisatropic $\tilde{\mathbf{K}}, \overrightarrow{\boldsymbol{J}}$ is parallel to $\overrightarrow{\boldsymbol{H}}$ only along three orthogonal axes of the specimen, which are called the principal axes of the tensor. If a specimen is characterized by isotropic $\tilde{\mathbf{K}}, \vec{J}$ is everywhere parallel to $\overrightarrow{\boldsymbol{H}}$, and $\tilde{\mathbf{K}}$ is reduced to a scalar quantity.

The nine components of the susceptibility tensor, $K_{i j}(i, j=1$, $2,3)$, may be expressed in terms of three equations relating the induced magnetization vector components along three orthogonal coordinate axes of the specimen to the external magnetic field vector components along these axes. If the coordinate axes are numbered 1, 2, and 3, corresponding, for example, to Cartesian coordinates, $\mathrm{X}_{1}, \mathrm{X}_{2}$, and $\mathrm{X}_{3}$, the three equations are

$$
\begin{aligned}
& J_{1}=K_{11} H_{1}+K_{12} H_{2}+K_{1 s} H_{s} \\
& J_{2}=K_{21} H_{1}+K_{22} H_{2}+K_{2 s} H_{s} \\
& J_{s}=K_{s 1} H_{1}+K_{s 2} H_{2}+K_{s s} H_{s}
\end{aligned}
$$

or, in briefer notation,

$$
J_{i}=\sum_{j=1}^{3} K_{i j} H_{j}, i=1,2,3 .
$$

Considerations of the principle of conservation of energy (Nye, 1960 , p. 57-60; Smythe, 1968, p. 21) require that

$$
K_{i j}=H_{j i}, i=1,2,3
$$

indicating that the susceptibility is a symmetric tensor which may be expressed by six components rather than nine.

By use of the formulation of Bhattacharya (1950), we may express the induced magnetization intensity in any direction having direction cosines $l_{l}, l_{2}$, and $l_{s}$, by

$$
J_{l}=\sum_{i=1}^{3} J_{i} l_{i}
$$

Combining equations 22 and 23 , we write

$$
J_{1}=\sum_{i=1}^{3} \sum_{j=1}^{3} l_{i} K_{i j} H_{j} .
$$

If $l_{1}, l_{2}$, and $l_{s}$ are taken to be the direction cosines of $\vec{H}$, we may write 


$$
H_{j}=l_{j} H, j=1,2,3
$$

Combining equations 24 and 25 , we write for the component of induced magnetization parallel to the external field

$$
J_{H}=H \sum_{i=1}^{3} \sum_{j=1}^{3} l_{i} l_{j} K_{i j} \text {. }
$$

The susceptibility component, $K_{H}$, parallel to the external field may be defined as the induced magnetization parallel to the field, divided by the field. Thus,

$$
K_{H}=\frac{J_{H}}{H}=\sum_{i=1}^{3} \sum_{j=1}^{3} l_{i} l_{j} K_{i j}
$$

which, expanded becomes

$$
K_{H}=l_{1}^{2} K_{11}+l_{2}^{2} K_{22}+l_{s}^{2} K_{s s}+2 l_{1} l_{2} K_{12}+2 l_{2} l_{s} K_{2 s}+2 l_{1} l_{s} K_{1 s}
$$

This component of susceptibility is of primary importance in susceptibility measuring systems, which are arranged to detect the induced magnetization component along the external magnetic field direction. $S_{i j}$

Just as the general second-degree equation, with coefficients

$$
\sum_{i=1}^{3} \sum_{j=1}^{3} S_{i j} X_{i} X_{j}=1
$$

possesses principal axes such that a transformation of axes allows us to write equation 27 as

$$
\sum_{i=1}^{3} S_{i} X_{i}{ }^{2}=1,
$$

so too does equation 26 have principal axes that allow us to rewrite the equation as

or, expanded, as

$$
\mathrm{K}_{H}=\sum_{i=1}^{3} l_{i}^{2} K_{i}
$$

$$
K_{H}=l_{1}^{2} K_{1}+l_{2}^{2} K_{q}+l_{s}^{2} K_{s} .
$$

Likewise, equation 22, which is the basic equation defining susceptibility, may be rewritten in terms of principal susceptibility components as

$$
J_{i}=K_{i} H_{i}, i=1,2,3 .
$$




\section{GEOMETRIC REPRESENTATIONS}

Tensor components of magnetic susceptibility may be represented in three-dimensional space by several mathematical surfaces. For example, one of the most direct representations of the important component, $K_{H}$, in equation 29 is the surface traced by a radius vector, $\vec{R}$, having a magnitude

$$
R=\frac{X_{i}}{l_{i}}=K_{H}, i=1,2,3
$$

where the $X_{i}$ are coordinates and the $l_{i}$ are directions cosines of $\overrightarrow{\boldsymbol{R}}$. If we substitute $R$ of equation 31 into equation 29, we have

$$
R^{3}=\sum_{i=1}^{3} X_{i}{ }^{2} K_{i}, i=1,2,3 .
$$

This equation is represented by the peanut-shaped surface shown in figure $6 A$. This surface may be referred to as the $K_{H}$ ovaloid.

A second representation of the susceptibility tensor may be obtained by comparing equation 29 with equation 28 , where the coefficients of $S_{1}$ are all positive in algebraic sign. Equation 28 is therefore a triaxial ellipsoid having its center at the origin of the $\mathrm{X}_{1}, \mathrm{X}_{2}$, and $\mathrm{X}_{3}$ coordinate system. If we take a radius vector, $\vec{R}$, parallel to $\overrightarrow{\boldsymbol{H}}$, having direction cosines $l_{1}, l_{2}$, and $l_{s}$, and extending from the origin to a point on the ellipsoidal surface, we may rewrite equation 28 as

$$
R^{2} \sum_{i=1}^{3} S_{i} l_{i}{ }^{2}=1
$$

If we rewrite equation 29 as

$$
\frac{1}{K_{H}} \sum_{i=1}^{3} K_{i} l_{i}^{2}=1,
$$

and take

$$
S_{i}=K_{i}
$$

and compare equations 33 and 34 , we see that $K_{H}$ may be represented by an ellipsoid traced out by a radius vector of length

$$
R=\frac{1}{\sqrt{K_{H}}} \text {. }
$$

This ellipsoidal surface is simpler in form than the $K_{H}$ ovaloid and is referred to as the representation quadric (Nye, 1960, p. 

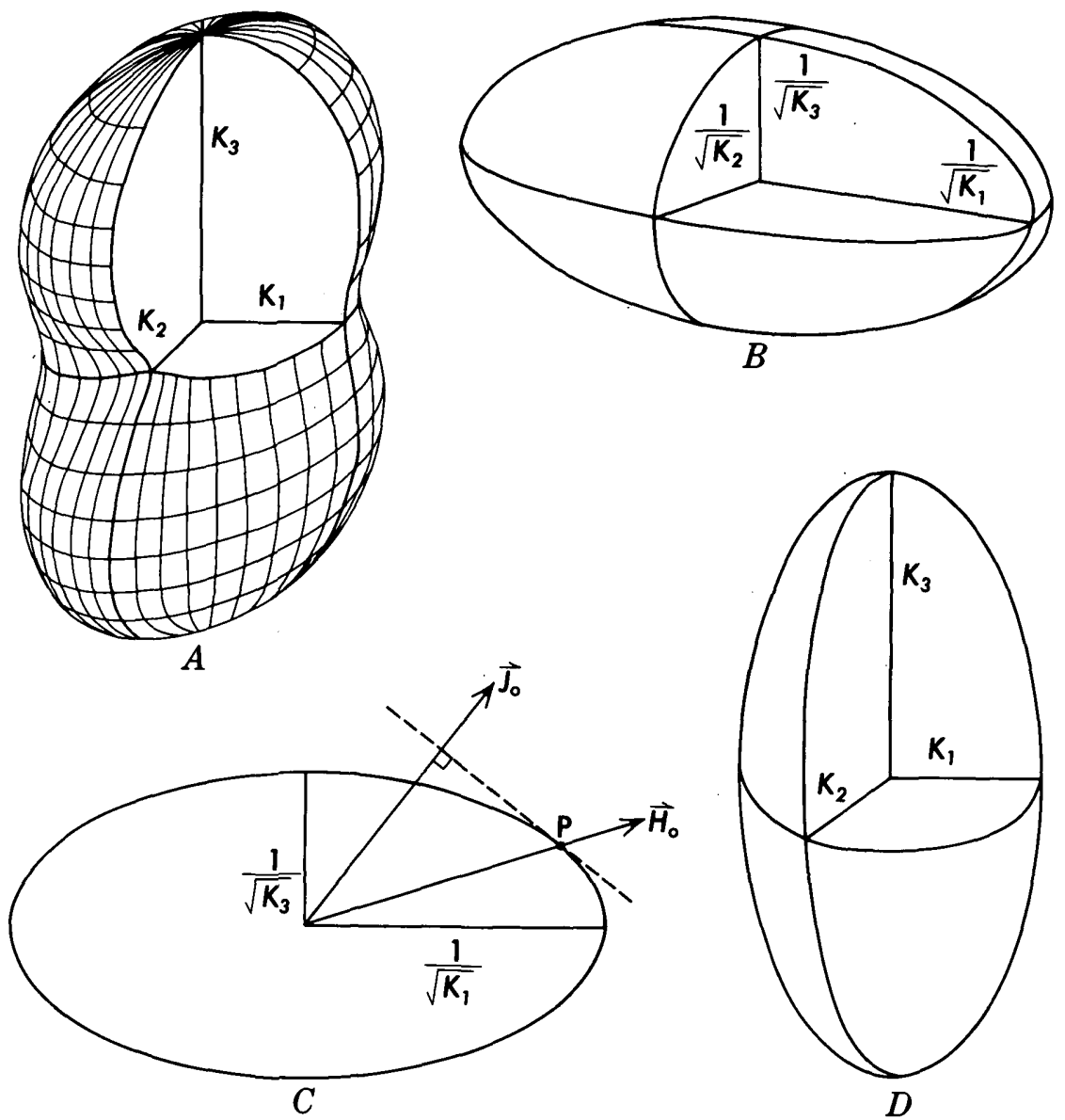

FIgURE 6.-Representations of susceptibility anisatropy. $A$. Perspective view of $\mathrm{K}_{\mathrm{H}}$ ovaloid. $B$. Perspective view of representation quadric. $C$. Cross section of principal plane of representation quadric, showing radius-normal property. If the external field vector, $\vec{H}_{\theta}$, intersects the ellipse at point $\mathrm{P}$, the resulting induced magnetization vector, $\vec{J}_{0}$, is normal to the tangent line at P. D. Perspective view of magnitude ellipsoid.

17). Along each coordinate axis, $X_{i}, \vec{R}$ takes the value

as shown in figure $6 B$.

$$
R_{i}=\frac{1}{\sqrt{K_{i}}}, i=1,2,3
$$

The representation quadric may be used not only to express the magnitude of $\mathrm{K}_{\mathrm{H}}$ in any direction, but also to indicate the direction of induced magnetization for a given external field direc- 
tion. This directional property may be demonstrated by considering the radius vector $\overrightarrow{\boldsymbol{R}}$, along the direction of $\overrightarrow{\boldsymbol{H}}$ (shown as $\overrightarrow{\boldsymbol{H}}_{o}$ in figure $6 C$ ), and having direction cosines $l_{1}, l_{2}$, and $l_{s}$, which extends from the origin to a point, $\mathrm{P}$, with coordinates $\left(R l_{1}, R l_{2}, R l_{s}\right)$ on the surface of the ellipsoid. The vector, $\vec{N}_{p}$, normal to the ellipsoidal surface at point $P$, is the gradient vector of the ellipsoidal function evaluated at point $P$ (see, for example, Kaplan, 1952). From equation 32 , the ellipsoidal function, $F\left(X_{i}\right)$, is

$$
\mathrm{F}\left(X_{i}\right)=\left(\sum_{i=1}^{3} K_{i} X_{i}^{2}\right)-K_{H}^{3}=0
$$

and the normal vector at $P$ is

$$
\vec{N}_{p}=\left.\nabla\left[\mathrm{F}\left(X_{i}\right)\right]\right|_{p}
$$

When we evaluate equation 36 , we have

$$
\begin{aligned}
\vec{N}_{\mathrm{p}} & =\left.\left(\vec{n}_{1} \frac{\partial}{\partial X_{1}}+\vec{n}_{2} \frac{\partial}{\partial X_{2}}+\vec{n}_{s} \frac{\partial}{\partial X_{s}}\right)\left(K_{1} X_{1}^{2}+K_{2} X_{2}^{2}+K_{s} X_{s}^{2}-K_{H}^{3}\right)\right|_{p} \\
& =\left(2 K_{1} X_{1}\right) \vec{n}_{1}+\left(2 K_{2} X_{2}\right) \vec{n}_{2}+\left.\left(2 K_{s} X_{s}\right) \vec{n}_{s}\right|_{p} \\
& =\left(2 K_{1} l_{1} R\right) \vec{n}_{1}+\left(2 K_{2} l_{2} \mathrm{R}\right) \vec{n}_{2}+\left(2 K_{s} l_{s} R\right) \vec{n}_{s}
\end{aligned}
$$

where $\vec{n}_{1}, \vec{n}_{2}$, and $\vec{n}_{s}$ are unit vectors along the $X_{1}, X_{2}$, and $X_{s}$ coordinates, and $R=\frac{1}{\sqrt{K_{H}}}$. We also note that the induced magnetization vector, $\vec{J}_{o}$ may be written from equation 22 , referred to principal axes, as

$$
\begin{aligned}
\overrightarrow{\boldsymbol{J}}_{o} & =\left(K_{1} H_{1}\right) \overrightarrow{\boldsymbol{n}}_{1}+\left(K_{2} H_{2}\right) \overrightarrow{\boldsymbol{n}}_{2}+\left(K_{s} H_{s}\right) \overrightarrow{\boldsymbol{n}}_{s} \\
& =\left(K_{1} l_{1} H\right) \overrightarrow{\boldsymbol{n}}_{1}+\left(K_{2} l_{2} H\right) \overrightarrow{\boldsymbol{n}}_{2}+\left(K_{s} l_{s} H\right) \overrightarrow{\boldsymbol{n}}_{s} .
\end{aligned}
$$

The induced magnetization vector, $\vec{J}_{o}$, is seen to be parallel to the normal vector $\vec{N}_{p}$, at point $\mathrm{P}$, by taking the cross product of the two vectors as follows

$$
\overrightarrow{\boldsymbol{J}}_{o} \times \vec{N}_{p}=\left|\begin{array}{ccc}
\overrightarrow{\boldsymbol{n}}_{1} & \overrightarrow{\boldsymbol{n}}_{2} & \overrightarrow{\boldsymbol{n}}_{s} \\
K_{1} l_{1} H & K_{2} l_{2} H & K_{s} l_{s} H \\
2 K_{1} l_{1} R & 2 K_{2} l_{2} R & 2 K_{s} l_{s} R
\end{array}\right|=0 .
$$

The fact that $\vec{J}_{o}$ is parallel to $\vec{N}_{p}$ is sometimes referred to as the radius-normal property of the representation quadric (Nye, 1960, p. 28). 
A third representation of magnetic susceptibility may be expressed by the surface traced out by the induced magnetization vector, $\overrightarrow{\boldsymbol{J}}$, for an external magnetic field of unit magnitude, or

$$
H=1 \text {. }
$$

When we rewrite equation 30 as

$$
J_{i}=K_{i} l_{i} H,
$$

square all terms

$$
J_{i}^{2}=K_{i}^{2} l_{i}^{2} H^{2}
$$

transpose terms, and sum $i$ from 1 to 3 , we have

$$
\sum_{i=l}^{3}\left(\frac{J_{i}}{H K_{i}}\right)^{2}=\sum_{i=1}^{3} l_{i}^{2}=1 .
$$

By using relation 37 , we may rewrite equation 38 as

$$
J^{2} \sum_{i=1}^{3}\left(\frac{l_{i}}{K_{i}}\right)^{2}=1
$$

where the $l_{i}$ are direction cosines of $\vec{J}$. We may compare equation 39 with the general equation for an ellipsoid referred to its principal axes and containing coefficients, $S_{i}$, which may be written

$$
\sum_{i=1}^{3}\left(\frac{X_{i}}{S_{i}}\right)^{2}=1,
$$

where the $S_{i}$ are the semiaxes of the ellipsoid. If the radius vector, $\vec{R}$, is taken parallel to $\vec{J}$, equation 40 may be expressed as

$$
R^{2} \sum_{i=1}^{3}\left(\frac{l_{i}}{S_{i}}\right)^{2}=1 .
$$

When we compare equations 39 and 41 and use relation 35 , we see that equation 39 is represented by an ellipsoid which has semiaxes of length $K_{1}, K_{2}$, and $K_{s}$ and which is traced out by a radius vector of length

$$
R=J \text {. }
$$

Because the radius vector is everywhere equal to $J$, assuming a unit external magnetic field, this surface is termed the magnitude ellipsoid (Nye, 1960, p. 47). It is shown in figure $6 D$.

The magnitude ellipsoid and representation quadric may be mutually derived from one another because the semiaxes of the 
latter are the reciprocal square root of the semiaxes of the former. These two surfaces are sometimes drawn concentrically to show in a single diagram both the magnitude and the direction of $\overrightarrow{\boldsymbol{J}}$ for a given $\overrightarrow{\boldsymbol{H}}$.

\section{CALCULATION OF PRINCIPAL SUSCEPTIBILITY COMPONENTS}

Magnetic susceptibility anisotropy is most conveniently expressed and geometrically represented by the magnitudes and directions of three principal susceptibility components rather than by the six components, $K_{i j}\left(i=1,2,3 ; K_{i j}=K_{j i}\right)$. The magnitudes and direction cosines $\left(l_{1}, l_{2}, l_{3}\right)$ of the principal susceptibilities may be obtained by using a generalized form of equation 30 , in which $K_{i}$ is taken to be $K$, and equation 22 combined as

$$
J_{i}=K H_{i}=\sum_{j=1}^{3} K_{i j} H_{j}, i=1,2,3
$$

or, by using relation 25 ,

$$
K l_{i}=\sum_{j=1}^{3} K_{i j} l_{j}, i=1,2,3 .
$$

When we expand equation 42 , we have

$$
\begin{aligned}
& \left(K_{11}-K\right) l_{1}+K_{12} l_{2}+K_{1 s} l_{s}=0 \\
& K_{12} l_{1}+\left(K_{22}-K\right) l_{2}+K_{2 s} l_{s}=0 \\
& K_{1 s} l_{1}+K_{2 s} l_{2}+\left(K_{s s}-K\right) l_{s}=0 .
\end{aligned}
$$

These homogeneous equations may be solved for $l_{1}, l_{2}$, and $l_{s}$, provided

$$
\left|\begin{array}{ccc}
\left(K_{11}-K\right) & K_{12} & K_{1 s} \\
K_{12} & \left(K_{22}-K\right) & K_{2 s} \\
K_{1 s} & K_{2 s} & \left(K_{s s}-K\right)
\end{array}\right|=0
$$

After expansion of the determinant, equation 44 is seen to be a cubic equation having three real roots, $K=K_{1}, K_{2}$, and $K_{3}$, which are the three principal susceptibilities. The directions of these principal susceptibilities, given by $l_{l}, l_{2}$, and $l_{s}$, may be determined by successively substituting the values $K_{1}, K_{2}$, and $K_{s}$ into equation 44.

\section{NONLINEAR SUSCEPTIBILITIES}

The assumption of linearity between magnetization and external field given by equation 22 is seldom, if ever, precisely observed in practice, either because the linear relationship is perturbed by experimental error or because the linear relationship 
itself is not valid. Where departures from linearity are known to fall within the range of expected experimental error, and where sufficient measurements are obtained to produce a redundancy of data, the observed data may be systematically adjusted to satisfy the linear relation. Graphically, this adjustment is analogous to fitting dispersed points of an $x-y$ plot to the best straight line defined in some sense, such as least squares. Analytically, the adjustment of data is equivalent to solving an overdetermined system of linear equations by minimizing residuals associated with experimental error, according to established procedures of numerical analysis (for comprehensive accounts, see Hamming, 1962, or Scheid, 1968). Analyses of errors involved in special types of anisotropic susceptibility measurements have been summarized by Stone (1967) and King (1967).

Where departures from linearity between magnetization and external field exceed expected experimental error, complex susceptibility anisotropies may be inferred. Nonlinear susceptibilities may theoretically occur as high-rank tensors that have components obeying special transformation laws (Saaty and Bram, 1964 ; Bloembergen, 1965), but more commonly they occur as nontensor quantities having no well-defined transformation laws.

A formal representation of nonlinear susceptibilities may be expressed by expanding the induced magnetization of a specimen in a power series in the external field, using the polyadic notation of J. W. Gibbs (Wilson, 1909),

$$
\overrightarrow{\boldsymbol{J}}=\tilde{\mathbf{K}} \cdot \overrightarrow{\boldsymbol{H}}+\tilde{\mathbf{K}}: \overrightarrow{\boldsymbol{H}} \overrightarrow{\boldsymbol{H}}+\tilde{\mathbf{K}}: \overrightarrow{\boldsymbol{H}} \overrightarrow{\boldsymbol{H}} \overrightarrow{\boldsymbol{H}}+\cdots
$$

The first term is a product of the linear susceptibility, a dyad, and the vector field. The second term is a product of the lowest order nonlinear susceptibility, a triad, and a dyad field term. The third term is a product of the next higher nonlinear susceptibility, a fourth-order polyad, and a triad field term. Rewritten in conventional subscript notation, equation 45 becomes

$$
J_{i}=\sum_{J=1}^{3} K_{i j} H_{j}+\sum_{j=1}^{3} \sum_{k=1}^{3} K_{i j k} H_{j} H_{k}+\sum_{j=1}^{3} \sum_{k=1}^{3} \sum_{l=1}^{3} K_{i j k l} H_{j} H_{k} H_{l}+\cdots
$$

or, in briefer notation,

$$
J_{i}=K_{i j} H_{j}+K_{i j k} H_{j} H_{k}+K_{i j k l} H_{j} H_{k} H_{l}+\cdots .
$$

The susceptibility $K_{i j}$ is the usual second-rank tensor, $K_{i j k}$, a thirdrank tensor, and $K_{i j k l}$, a fourth-rank tensor. As nonlinear tensoral susceptibilities become important only in relatively strong fields, they are not expected to contribute significantly to complex 
anisotropies observed in weak-field measurements. Nor, as previously noted, is the effect of magnetocrystalline anisotropy of magnetite expected to contribute to the complexity of weak-field anisotropies.

The most likely causes of complicated weak-field anisotropy in magnetite-bearing rocks appear to be highly irregular or uneven volume distributions of magnetite grains within rocks which contain a relatively small number of low-coercivity grains. If the number of grains per unit volume of rock is small, the statistical averaging effect of susceptibility anisotropies of dispersed grains is small. As the present report is limited to the measurement of linear susceptibilities, no attempt will be made to treat determination of nonlinear susceptibilities.

\section{ANISOTROPY MEASUREMENTS USING A SPINNER MAGNETOMETER}

\section{PRINCIPLES OF MEASUREMENT}

Because the susceptibility tensor may be represented by a surface-such as the magnitude ellipsoid-having three principal axes, it is reasonable to suppose that the tensor may be measured by determining the projections of these axes on three orthogonal planes. This procedure is analogous to measuring a remanent magnetization vector by determining its components projected on orthogonal planes. As remanent magnetization vector components may be effectively determined by rotating a rock sample in the vicinity of a pickup coil, this measurement procedure seems appropriate for measuring components of the anisotropic susceptibility tensor.

If a rock sample possessing anisotropic susceptibility is rotated about an axis near a pickup coil, the voltage induced in the coil is proportional to the time rate of change of the magnetic induction component parallel to the coil axis, integrated over the area of the coil loops (see, for example, Maxwell, 1892; Stratton, 1941). Further, the magnetic induction associated with the rock sample is proportional to the induced magnetization, and therefore to the susceptibility, of the rock sample. ${ }^{7}$ If the susceptibility components, $K_{i j}(i, j=1,2,3)$, are referenced to the

\footnotetext{
7 According to Maxwell's equations for large-scale electromagnetic phenomena (Maxwell, 1892: Stratton, 1941), the magnetastatic field giving rise to an electromotive force in the pickup coil may be generated by (1) a time rate of change of electric field intensity (electrostatic field) associated with electric charge or electric polarization, (2) electric current (conductive, rather than displacement), (3) a time rate of change of electric polarization, and (4) magnetization. In addition to the intrinsic magnetic susceptibility and the demagnetizing factor, second-rank tensors associated with the rock specimen (and, therefore the generated electromotive force) may include electric permittivitv (capacivity), magnetic permeability, electric conductivity or resistivity, electric susceptibility, electric depolarizing factor, and remanent magnetic or electric quadrupole moments.
} 

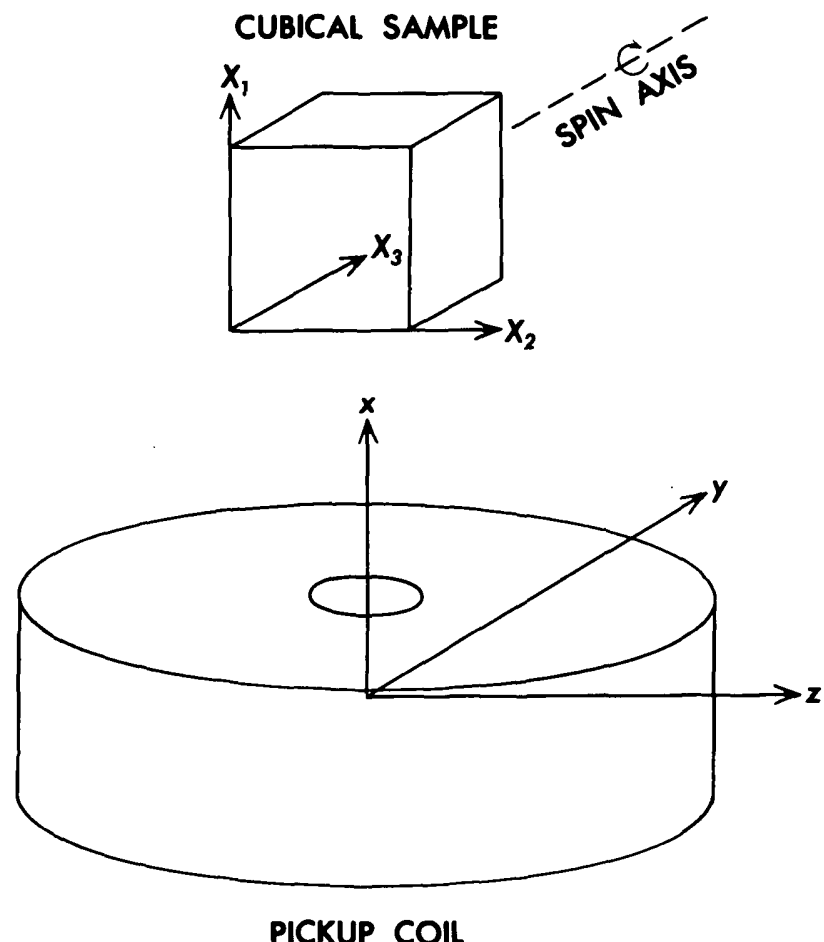

FIGURE 7.-Relationship between the coordinates of the rotating rock sample, $X_{1}, X_{2}$, and $X_{3}$, and the stationary coordinates of the pickup coil, $x, y$, and $z$.

coordinate system of the sample $\left(X_{1}, X_{2}, X_{s}\right)$, and the sample is rotated about the $X_{s}$ axis relative to the pickup coil coordinate system, $x, y, z$, where $z$ coincides with $X_{s}$, and $X_{1}$ coincides with the coil axis (fig. 7 ), we may write

$$
\epsilon_{x} \propto \frac{\mathrm{d} J_{x}}{\mathrm{~d} t}
$$

or

$$
\epsilon_{x} \propto \frac{\mathrm{d}}{\mathrm{d} t}\left(K_{x x} H_{x}+K_{x y} H_{y}+K_{x z} H_{z}\right),
$$

where $\epsilon_{x}$ is the voltage or electromotive force induced in the coil. The susceptibility components, $K_{x x}, K_{x y}$, and $K_{x z}$, may be expressed in the terms of the $K_{i j}$ sample coordinates or a set of $K_{i j}$ coordinates which are referred to the principal susceptibilities in the plane normal to the rotation axis (fig. 8) by using transformation equations involving orthogonal matrices.

If the sample coordinate system is rotated clockwise by an angle, $\theta$, about the $X_{s}$ axis, which coincides with the $z$ axis, the susceptibility components $K_{i j}(i, j=x, y, z)$ may be expressed in 


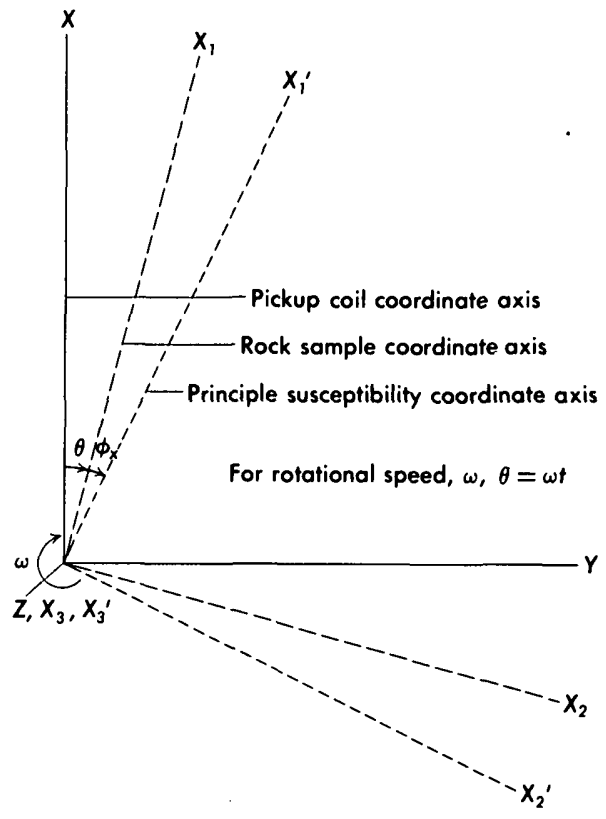

Figure 8.-Relationship among the coordinate axes of the pickup coil, the rotating rock sample, and the principal susceptibility components in the $X_{1}-X_{2}$ plane, normal to the rotation axis. Coordinate axes $Z, X_{3}$, and $X_{3}^{\prime}$ are directed along the rotation axis, into the plane of the diagram.

terms of the $K_{i j}(i, j=1,2,3)$ components by the matrix equation

$$
\begin{array}{r}
\left(\begin{array}{ccc}
\cos \theta & \sin \theta & 0 \\
-\sin \theta & \cos \theta & 0 \\
0 & 0 & 1
\end{array}\right)\left(\begin{array}{l}
K_{11} K_{12} K_{1 s} \\
K_{12} K_{22} K_{2 s} \\
K_{1 s} K_{2 s} K_{s s}
\end{array}\right)\left(\begin{array}{ccc}
\cos \theta & -\sin \theta & 0 \\
\sin \theta & \cos \theta & 0 \\
0 & 0 & 1
\end{array}\right) \\
= \\
=\left(\begin{array}{l}
K_{x x} K_{x y} K_{x z} \\
K_{x y} K_{y y} K_{y z} \\
K_{x z} K_{y z} K_{z z}
\end{array}\right) .
\end{array}
$$

If we take the matrix product on the left-hand side of equation 47 and equate elements of the product to matrix elements on the right-hand side of the equation, we have

$$
\begin{aligned}
& K_{x x}=K_{11} \cos ^{2} \theta+K_{22} \sin ^{2} \theta+2 K_{12} \sin \theta \cos \theta, \\
& K_{y y}=K_{11} \sin ^{2} \theta+K_{22} \cos ^{2} \theta-2 K_{12} \sin \theta \cos \theta, \\
& K_{z z}=K_{s s} \\
& K_{x y}=\left(K_{22}-K_{11}\right) \sin \theta \cos \theta+K_{12}\left(\cos ^{2} \theta-\sin ^{2} \theta\right), \\
& K_{x z}=K_{1 s} \cos \theta+K_{2 s} \sin \theta,
\end{aligned}
$$

and

$$
K_{y z}=-K_{1 s} \sin \theta+K_{2 s} \cos \theta .
$$


When we use trigonometric identities for double angles, we may rewrite $K_{x x}$ and $K_{x y}$ as

and

$$
K_{x x}=\frac{K_{11}}{2}(1+\cos 2 \theta)+\frac{K_{22}}{2}(1-\cos 2 \theta)+K_{12} \sin 2 \theta,
$$

$$
K_{x y}=\frac{\left(K_{22}-K_{11}\right)}{2} \sin 2 \theta+K_{12} \cos 2 \theta .
$$

If the $X_{s}$ spin axis has a rotational velocity, $\omega$, we may write $\theta$ as a function of time, $t$, as

$$
\theta=\omega t \text {. }
$$

If we substitute $K_{x x}, K_{x y}$, and $K_{x z}$ into equation 46 , we have

$$
\begin{aligned}
\epsilon_{x} \propto \frac{\mathrm{d}}{\mathrm{d} t}\{[1 / 2 & \left.\left(K_{11}+K_{22}\right)+1 / 2\left(K_{11}-K_{22}\right) \cos 2 \omega t+K_{12} \sin 2 \omega t\right] H_{x} \\
+ & {\left[1 / 2\left(K_{22}-K_{11}\right) \sin 2 \omega t+K_{12} \cos 2 \omega t\right] H_{y} } \\
+ & {\left.\left[K_{1 s} \cos \omega t+K_{2 s} \sin \omega t\right] H_{z}\right\} . }
\end{aligned}
$$

From equation 49 , we see that the external field components, $H_{x}$ and $H_{y}$, normal to the rotation axis, give rise to alternating voltages that have frequencies twice that of the rotational velocity, whereas the external field component, $H_{z}$, parallel to the rotation axis, gives rise to a voltage having the same frequency as the rotational velocity.

The signal generated by the anisotropic susceptibility of a rotating rock sample may be considered in greater detail by referencing the susceptibility components to coordinates $X_{1}^{\prime}, X_{2}^{\prime}$, and $X_{s}^{\prime}$, of which $X^{\prime}$, and $X_{2}^{\prime}$ are the directions of principal susceptibilities in the plane normal to the rotation axis, and $X^{\prime}$, is the direction of the rotation axis. From figure 8 , we see that transformation of coordinate axes corresponding to the rock sample and pickup coil has the same form as the transformation of axes corresponding to the principal susceptibilities and pickup coil, except that the angle of rotation is increased from $\theta=\omega t$ to $\theta^{\prime}=\omega t+$ $\phi_{x}$, where $\phi_{x}$ is the angle between the principal susceptibility in the vertical plane and the corresponding pickup coil coordinate axis. Using orthogonal matrices similar to those of equation 47 and noting that $K_{12}^{\prime}=0$ when referred to the principal susceptibility coordinates, we have

$$
\begin{gathered}
\left(\begin{array}{ccc}
\cos \left(\omega t+\phi_{x}\right) \sin \left(\omega t+\phi_{x}\right) & 0 \\
-\sin \left(\omega t+\phi_{x}\right) \cos \left(\omega t+\phi_{x}\right) & 0 \\
0 & 0 & 1
\end{array}\right)\left(\begin{array}{ccc}
K_{11}^{\prime} & 0 & K_{1 s}^{\prime} \\
0 & K_{2,}^{\prime} & K_{2 s}^{\prime} \\
K_{1 s}^{\prime} & K_{2 s}^{\prime} & K_{s s}^{\prime}
\end{array}\right) \\
\left(\begin{array}{ccc}
\cos \left(\omega t+\phi_{s}\right) & -\sin \left(\omega t+\phi_{x}\right) & 0 \\
\sin \left(\omega t+\phi_{x}\right) & \cos \left(\omega t+\phi_{x}\right) & 0 \\
0 & 0 & 1
\end{array}\right)-\left(\begin{array}{l}
K_{r r} K_{r y} K_{x z} \\
K_{x y} K_{y y} K_{y z z} \\
K_{x z} K_{y z} K_{z z}
\end{array}\right) .
\end{gathered}
$$


The equation for induced voltage, corresponding to equations 48 and 49 , is

$$
\begin{aligned}
\epsilon_{x} \propto \frac{\mathrm{d}}{\mathrm{d} t} & \left\{\left[1 / 2\left(K_{11}^{\prime}+K_{22}^{\prime}\right)+1 / 2\left(K_{11}^{\prime}-K_{22}^{\prime}\right) \cos 2\left(\omega t+\phi_{x}\right)\right] H_{x}\right. \\
& +\left[1 / 2\left(K_{22}^{\prime}-K_{11}^{\prime}\right) \sin 2\left(\omega t+\phi_{x}\right)\right] H_{y} . \\
& \left.+\left[K_{1 s}^{\prime} \cos \left(\omega t+\phi_{x}\right)+K_{2 s}^{\prime} \sin \left(\omega t+\phi_{x}\right)\right] H_{z}\right\}
\end{aligned}
$$

or

$$
\begin{aligned}
& \epsilon_{x} \propto\left[\omega\left(K_{22}^{\prime}-K_{11}^{\prime}\right) \sin 2\left(\omega t+\phi_{x}\right)\right] H_{x} \\
& \quad+\left[\omega\left(K_{22}^{\prime}-K_{11}^{\prime}\right) \cos 2\left(\omega t+\phi_{x}\right)\right] H_{y} \\
& \quad+\left[-\omega K_{13}^{\prime} \sin \left(\omega t+\phi_{x}\right)+\omega K_{23}^{\prime} \cos \left(\omega t+\phi_{x}\right)\right] H_{z} .
\end{aligned}
$$

If we combine the $H_{x}$ and $H_{y}$ terms, we may rewrite equation 51 as

$$
\begin{aligned}
\epsilon_{x} \propto\left\{\omega \left(K_{22}^{\prime}-\right.\right. & \left.\left.K_{11}^{\prime}\right) \sin \left[2\left(\omega t+\phi_{x}\right)+\tan ^{-1}\left(\frac{H_{y}}{H_{x}}\right)\right]\right\} \sqrt{H_{x}^{2}+H^{2}}{ }_{\nu} \\
& +\left\{-\omega K_{1 s}^{\prime} \sin \left(\omega t+\phi_{x}\right)+\omega K_{2 s}^{\prime} \cos \left(\omega t+\phi_{x}\right)\right\} H_{z} .
\end{aligned}
$$

The voltage associated with external field component $H_{z}$ may be cancelled by electrical filtering or by nulling the $H_{z}$ component during the measurement process. Ordinarily, only the external field component, $H_{x}$, is used during the measurement process, so that equation 52 becomes

$$
\epsilon_{x} \propto\left[\omega\left(K_{22}^{\prime}-K_{11}^{\prime}\right) \sin 2\left(\omega t+\phi_{x}\right)\right] H_{x} .
$$

The amplitude of the alternating emf is thus proportional to the principal susceptibility difference, $\left(K_{22}^{\prime}-K_{11}^{\prime}\right)$, the rotational velocity, $\omega$, and the external field component, $H_{r .}$. The frequency of the sinusoidal voltage is $2 \omega t$, that is, twice the rotational frequency of the rotation axis, and the observed phase angle is twice the angle between the principal susceptibility axis and the corresponding rock sample coordinate axis. The angular velocity, $\omega$, and the external field component, $H_{x}$, are known quantities in the measurement process, and they may be incorporated into a lumped coefficient giving the proportionality factor between the voltage amplitude, $\epsilon_{x}$, and the suisceptibility difference, $\left(K_{22}^{\prime}-\right.$ $K_{11}^{\prime}$ ) This lumped coefficient may include design factors associated with coil construction (Collinson and Creer, 1960, p. 190-194) and constants obtained by calibration of the measuring apparatus. Thus, equation 53 may be rewritten in terms of the observed voltage amplitude

$$
\epsilon_{x}=\xi\left(K_{11}^{\prime}-K_{22}^{\prime}\right) \text {, }
$$


where $\xi$ is the lumped proportionality factor previously determined. If $A$ is the adjusted observed voltage amplitude, such that

we may write

$$
A=\frac{\epsilon_{x}}{\xi},
$$

$$
A=K_{11}^{\prime}-K_{22}^{\prime} \text {. }
$$

The observed phase angle, $\phi_{x}$, for this axis orientation is called $\alpha$, that is

$$
\alpha=\phi_{x} .
$$

Similarly, if the rock sample is rotated about the $X_{1}$ and $X_{2}$ sample coordinate axes, we substitute for the left-hand side of equation 50 the matrix products

$$
\begin{aligned}
\left(\begin{array}{ccc}
\cos \left(\omega t+\phi_{y}\right) & 0 \sin \left(\omega t+\phi_{y}\right) \\
0 & 1 & 0 \\
-\sin \left(\omega t+\phi_{y}\right) & 0 \cos \left(\omega t+\phi_{y}\right)
\end{array}\right)\left(\begin{array}{ccc}
K_{11}^{\prime} K_{12}^{\prime} 0 \\
K_{12}^{\prime} K_{22}^{\prime} K_{2 s}^{\prime} \\
0 K_{2 s}^{\prime} K_{s s}^{\prime}
\end{array}\right) & \left(\begin{array}{ccc}
\cos \left(\omega t+\phi_{y}\right) & 0-\sin \left(\omega t+\phi_{y}\right) \\
0 & 1 & 0 \\
\sin \left(\omega t+\phi_{y}\right) & 0 & \cos \left(\omega t+\phi_{y}\right)
\end{array}\right)
\end{aligned}
$$

and

$$
\begin{aligned}
\left(\begin{array}{ccc}
1 & 0 & 0 \\
0 & \cos \left(\omega t+\phi_{z}\right) & \sin \left(\omega t+\phi_{z}\right) \\
0 & -\sin \left(\omega t+\phi_{z}\right) & \cos \left(\omega t+\phi_{z}\right)
\end{array}\right)\left(\begin{array}{ll}
K_{11}^{\prime} K_{12}^{\prime} K_{1 s}^{\prime} \\
K_{12}^{\prime} K_{22}^{\prime} 0_{1 s} \\
K_{1 s}^{\prime} 0^{\prime} K_{s s}^{\prime}
\end{array}\right) & \\
& \left(\begin{array}{ccc}
1 & 0 & 0 \\
0 & \cos \left(\omega t+\phi_{z}\right) & -\sin \left(\omega t+\phi_{z}\right) \\
0 & \sin \left(\omega t+\phi_{z}\right) & \cos \left(\omega t+\phi_{z}\right)
\end{array}\right) .
\end{aligned}
$$

If the phase angles are measured, in order, from $y$ to $x, x$ to $z$, and $z$ to $y$ (fig. 9), we have

and

$$
\begin{aligned}
& B=\left(K_{22}^{\prime}-K_{s s}^{\prime}\right), \\
& \beta=\phi_{y y} \\
& C=\left(K_{s s}^{\prime}-K_{11}^{\prime}\right),
\end{aligned}
$$

$$
\gamma=\phi_{z} .
$$

Given the measurements $A, B, C, \alpha, \beta$, and $\gamma$, we now inquire about the relationship between the measured amplitudes and phase angles to the desired susceptibility components $K_{i j}(i, j=1$, $2,3)$. To obtain this relationship, we construct the following matrix equations 

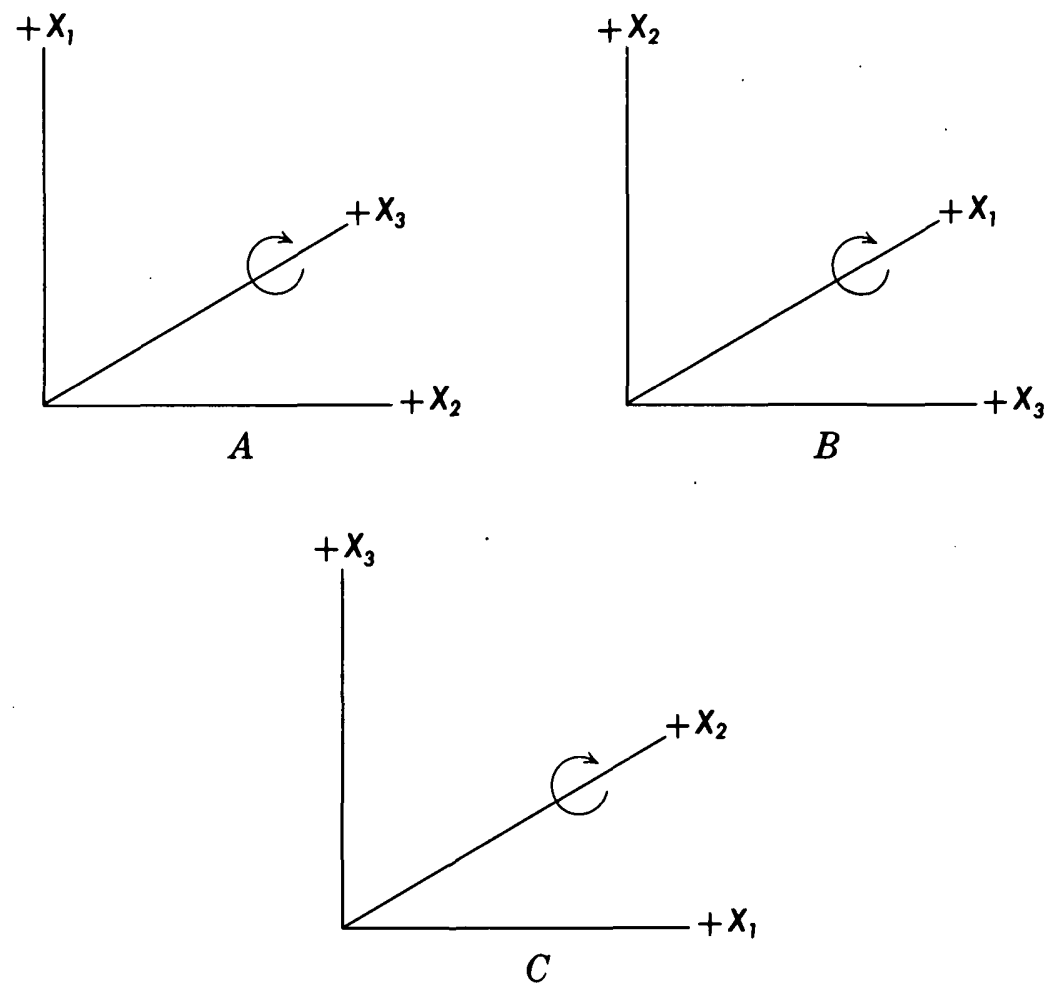

Figure 9.-Sequential order of orientations of rock sample coordinate system during the measurement process. (A) First spin position. (B) Second spin position. (C) Third spin position.

$$
\begin{aligned}
& \left(\begin{array}{ccc}
\cos \phi_{x} \sin \phi_{x} & 0 \\
-\sin \phi_{x} \cos \phi_{x} & 0 \\
0 & 0 & 1
\end{array}\right)\left(\begin{array}{ccc}
K_{11}^{\prime} & 0 & K_{1 s}^{\prime} \\
0 & K_{22}^{\prime} & K_{2 s}^{\prime} \\
K_{1 s}^{\prime} & K_{2 s}^{\prime} & K_{s s}^{\prime}
\end{array}\right)\left(\begin{array}{ccc}
\cos \phi_{x} & -\sin \phi_{x} & 0 \\
\sin \phi_{x} & \cos \phi_{x} & 0 \\
0 & 0 & 1
\end{array}\right) \\
& =\left(\begin{array}{l}
K_{11} K_{12} K_{1 s} \\
K_{12} K_{22} K_{2 s} \\
K_{1 s} K_{2 s} K_{s s}
\end{array}\right)
\end{aligned}
$$

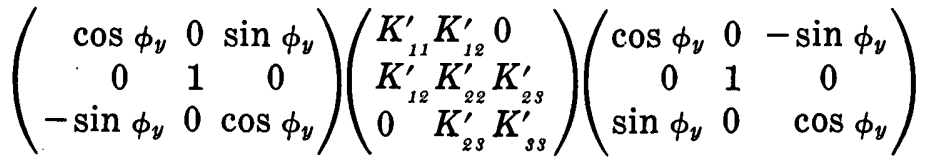

$$
\begin{aligned}
& =\left(\begin{array}{l}
K_{11} K_{12} K_{1 s} \\
K_{12} K_{22} K_{2 s} \\
K_{1 s} K_{2 s} K_{s s}
\end{array}\right) \text {, }
\end{aligned}
$$


and

$$
\begin{array}{r}
\left(\begin{array}{ccc}
1 & 0 & 0 \\
0 & \cos \phi_{z} \sin \phi_{z} \\
0 & -\sin \phi_{z} \cos \phi_{z}
\end{array}\right)\left(\begin{array}{ll}
K_{11}^{\prime} K_{12}^{\prime} K_{1 s}^{\prime} \\
K_{12}^{\prime} K_{22}^{\prime} 0 \\
K_{1 s}^{\prime} K_{1 s}^{\prime} & K_{s s}^{\prime}
\end{array}\right)\left(\begin{array}{ccc}
1 & 0 & 0 \\
0 & \cos \phi_{z} & -\sin \phi_{z} \\
0 & \sin \phi_{z} & \cos \phi_{z}
\end{array}\right) \\
=\left(\begin{array}{c}
K_{11} K_{12} K_{1 s} \\
K_{12} K_{22} K_{2 s} \\
K_{1 s} K_{2 s} K_{s s}
\end{array}\right) .
\end{array}
$$

From equation 54 we obtain

$$
\begin{aligned}
& K_{11}=1 / 2\left(K_{11}^{\prime}+K_{22}^{\prime}\right)+1 / 2\left(K_{11}^{\prime}-K_{22}^{\prime}\right) \cos 2 \phi_{x}, \\
& K_{22}=1 / 2\left(K_{11}^{\prime}+K_{22}^{\prime}\right)-1 / 2\left(K_{11}^{\prime}-K_{22}^{\prime}\right) \cos 2 \phi_{x},
\end{aligned}
$$

and

$$
K_{12}=-1 / 2\left(K_{11}^{\prime}-K_{22}^{\prime}\right) \sin 2 \phi_{x} .
$$

When we take the difference of the first two equations of system 57, we obtain

$$
K_{11}-K_{22}=\left(K_{11}^{\prime}-K_{22}^{\prime}\right) \cos 2 \phi_{x} .
$$

Thus, from relations 57 and 58

$$
K_{11}-K_{22}=A \cos 2 \alpha,
$$

and

$$
K_{12}=-1 / 2 A \sin 2 \alpha .
$$

Likewise, when using equations 55 and 56 , we obtain

$$
\begin{aligned}
K_{22}-K_{s s} & =B \cos 2 \beta, \\
K_{2 s} & =-1 / 2 B \sin 2 \beta, \\
K_{s s}-K_{11} & =C \cos 2 \gamma,
\end{aligned}
$$

and

$$
K_{1 s}=-1 / 2 C \sin 2 \gamma .
$$

As seen from equations 59 and 60 , the cross susceptibility components, $K_{12}, K_{2 s}$, and $K_{1 s}$, are completely determined in the measurement process, whereas the normal susceptibility components, $K_{11}, K_{22}$, and $K_{s s}$, are determined only to within an unknown constant. Thus, in order to completely specify the susceptibility tensor, it is necessary to measure one of the normal susceptibility components independently. Without this measurement, the observed spinner magnetometer data are limited to determinations of the differences of principal susceptibilities rather than to determinations of the principal susceptibility values themselves. The orientations of principal susceptibility axes may be determined from the spinner magnetometer data alone. 


\section{INDEPENDENT MEASUREMENT OF NORMAL SUSCEPTIBILITY COMPONENT}

Although differences of principal susceptibilities may adequately describe susceptibility anisotropy in some types of rock magnetic investigations, determinations of individual principal susceptibility components are necessary where complete determinations of anisotropy are required. Complete determination of principal susceptibility values is possible only if the normal susceptibility components, $K_{11}, K_{22}$, and $K_{s s}$, are known. Equations 59 and 60 indicate that independent determination of one of these normal susceptibilities is sufficient to determine the other two.

A straightforward method of determining one of the normal susceptibilities is by orienting one of the rock sample coordinates parallel to an external magnetic field and measuring the induced magnetization of the sample in that direction. This measurement may be conveniently made by using a commercially available susceptibility bridge or a similar apparatus that uses an alternating external field. Consistency is achieved if the frequency of the external field is twice the rotational frequency of the spinner magnetometer used to determine the other susceptibility components.

'If, for example, the measured induced magnetization along the $X_{s}$ sample coordinate, $J_{s}^{*}$, is associated with an applied external field, $H_{s}^{*}$, in that same direction, the normal susceptibility component is

$$
K_{s s}=K_{s s}^{*}=\frac{J_{s}^{*}}{H_{s}^{*}}
$$

By substituting in equations 59 and 60 , we have for values of normal susceptibilities

and

$$
\begin{aligned}
& K_{11}=A \cos 2 \alpha+B \cos 2 \beta+K_{s s}^{*}, \\
& K_{22}=B \cos 2 \beta+K_{s s}^{*},
\end{aligned}
$$

$$
K_{s s}=K_{s s}^{*} .
$$

These values, along with those for cross susceptibilities, $K_{12}, K_{18}$, and $K_{2 s}$, may be substituted into equation 44 to obtain the principal susceptibilities, $K_{1}, K_{2}$, and $K_{s}$.

The direct measurement of $K_{s s}$ by using a bridge or similar apparatus suggests the possibility of directly measuring each of the normal susceptibilities by this method and reserving the 
spinner magnetometer data for determinations of cross susceptibilities and orientations of principal susceptibility axes. However, for a detection system of given sensitivity, measurements of small differences of susceptibilities can be made more accurately than determinations of these differences by subtracting independently measured values of susceptibilities. Therefore, all of the signal observed in the spinner magnetometer measurement is directly related to anisotropy, whereas often less than 1 percent of the signal observed in the bridge measurement is associated with anisotropy.

\section{METHODS OF EXPRESSING ANISOTROPY}

The most direct way of expressing linear susceptibility anisotropy is by specifying the six components, $K_{11}, K_{22}, K_{s s}, K_{12}, K_{1 s}$, and $K_{2 s}$, or, equivalently, by expressing the principal susceptibilities, $K_{1}, K_{2}$, and $K_{s}$ in combination with the three angles associated with orientations of principal susceptibility axes relative to a fixed coordinate system of the rock specimen. ${ }^{8}$ In addition to these direct expressions, other quantities have been defined by various authors to indicate the magnitude of anisotropy of a rock. Among the more common expressions are the following:

$\{1\}$ Magnetic lineation, $L$, where

$$
L=\frac{K_{\max }-K_{\min }}{\bar{K}},
$$

\{2\} Magnetic foliation, $F$, where

$$
F=\frac{K_{\text {int }}-K_{\min }}{\bar{K}}
$$

\{3\} Prolateness $=\frac{L}{F}$,

$\{3\}$ Oblateness $=\frac{F}{L}$,

where

$$
\bar{K}=\frac{K_{\max }+K_{\text {int }}+K_{\min }}{3}
$$

sometimes termed $K_{B}$, the bulk susceptibility,

\{5\} Maximum anisotropy

$$
=\frac{K_{\max }-K_{\min }}{\bar{K}},
$$

\footnotetext{
${ }^{8}$ A complete description of the anisotropy actually requires a determination of only five quantities, either five susceptibility tensor components, or a combination of three principal susceptibilities and two angles indicating the orientation of principal axes.
} 
\{6\} Degree of anisotropy, $\mathrm{P}(\max / \mathrm{min})$

$$
\frac{K_{\max }}{K_{\min }}
$$

\{7\} Degree of anisotropy, $P(\max /$ int)

$$
=\frac{K_{\text {max }}}{K_{\text {int }}},
$$

\{8\} Degree of anisotropy, $\mathrm{P}$ (int/min)

\{9\} Anisotropy

$$
=\frac{K_{\text {int }}}{K_{\text {min }}} \text {, }
$$

$$
=\frac{K_{\max }+K_{\text {int }}}{2 K_{\min }},
$$

\{10\} Magnetic anisotropy ratio

$$
=\frac{K_{\text {par }}}{K_{\text {per }}}
$$

where $\mathrm{K}_{\text {par }}$ is measured for magnetic field parallel to rock layering and $\mathrm{K}_{\mathrm{per}}$ is measured for magnetic field perpendicular to rock layering,

\{11\} Excess of susceptibility of the magnetic stratum plane, $\bar{h}$, where

$$
\bar{h}=\frac{K_{\max }+K_{\text {int }}}{2}-K_{\min }, \text { and }
$$

\{12\} Azimuthal anisotropy quotient,

$$
q=\frac{K_{\max }-K_{\min }}{\bar{h}} .
$$

Expressions 1, 2, 3, and 4 have been used by King and Rees (1962) and Khan (1962); they are similar to definitions by F. D. Stacey (Stacey, Joplin, and Lindsay, 1960) which were based on demagnetization factor components. These expressions convey information analogous to ratios of $K_{m a x} / K_{i n t} / K_{m i n}$, which have been rationalized by dividing by $K_{\text {int }}$. Ratios of $K_{m a x} / K_{\text {int }} / K_{\text {min }}$, rationalized by dividing by $K_{m i n}$, have been used by Girdler (1961a). Expression 5 was adopted by Howell, Martinez, and Statham (1958), and expressions 6, 7, and 8, by Uyeda, Fuller, Belshé, and Girdler (1963). Expression 9 was used by Balsley and Buddington (1960) and expression 10 by Jahren (1963). Expressions 
11 and 12, defined by Granar (1959), are extensions of definitions first given by Isling (1943). Hamilton and Rees (1965) considered $K_{\text {int }}$ to represent approximately the mean susceptibility, $\left(K_{\max }-K_{\min }\right) / K_{\text {int }}$, the intensity of foliation in terms of mean susceptibility, and $q$ of expression 12, the intensity of lineation in terms of that of the foliation. If the susceptibility magnitude ellipsoid assumes the shape of an oblate or prolate spheroid, the anisotropy may be specified by the ratio of maximum-to-minimum susceptibility, equivalent to the anisotropy factor used by Uyeda, Fuller, Belshé, and Girdler (1963).

A convenient expression of anisotropy suitable for use with the spinner magnetometer, an apparatus by which differences of susceptibilities rather than susceptibilities themselves are measured, is expression. 5. This definition is adopted in this report and modified to indicate a percent rather than a pure number. The term is given as

$$
\text { Maximum percent anisotropy }=\left(\frac{K_{m a x}-K_{m i n}}{K_{b u l k}}\right) 100 \text { percent }
$$

where

$$
\begin{aligned}
K_{b u l i} & =1 / 3\left(K_{11}+K_{22}+K_{s s}\right) \\
& =1 / 3\left(K_{1}+K_{2}+K_{s}\right) .
\end{aligned}
$$

\section{MEAD-TYPE SPINNER MAGNETOMETER}

A particular type of spinner apparatus used successfully in the early 1960's to measure susceptibility anisotropy was designed by Professor Judson Mead of Indiana University (Hanna, 1965; Hanna and Mead, 1968) and is herein referred to as the Mead-type spinner magnetometer. The basic component of the apparatus, which is shown schematically in figure 10, is a motordriven spinner magnetometer (operated at a rotational speed of $600 \mathrm{rpm}$ ) ordinarily used to measure the $10-\mathrm{Hz}$ signal associated with the remanent magnetization of a rock specimen. For purposes of measuring susceptibility anisotropy, an auxiliary horizontal shaft is geared to the main shaft in a $1: 2$ ratio so that the auxiliary shaft rotates at $300 \mathrm{rpm}$, or half the speed of the main shaft. A vertically oriented Helmholtz coil is placed in the region of the sample holder (which is attached to the auxiliary shaft) to provide a fixed uniform external magnetic field. When a rock sample is rotated at $300 \mathrm{rpm}$, the $10-\mathrm{Hz}$ signal generated by the anisotropic susceptibility normal to the shaft is amplified, filtered, and detected by the pickup. system of the basic spinner 


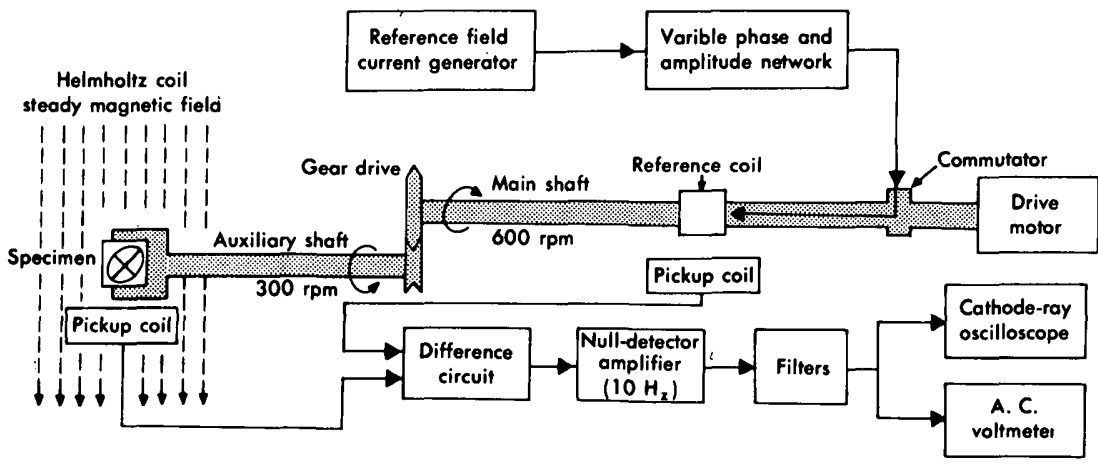

Figure 10.-Mead-type spinner magnetometer used for measuring magnetic susceptibility anisotropy. The pickup and detection system, consisting of components shown below the rotating shafts in the diagram, is also used for measuring remanent magnetization of a rotating sample and magnetic susceptibility of a stationary sample.

magnetometer. For purposes of independently measuring the magnetic susceptibility along a particular rock specimen coordinate (to completely specify the susceptibility ellipsoid), the Helmholtz coil may be used to produce a $10-\mathrm{Hz}$ alternating field surrounding the rock which is held stationary, so that the $10-\mathrm{Hz}$ pickup system of the spinner magnetometer may again be used. An external field of two oersteds, which is sufficiently weak to insure linearity between magnetization intensity and magnetic field (see, for example, Nagata, 1961), is used for both the anisotropic susceptibility measurements of the rotating specimen and the apparent susceptibility measurement of the stationary specimen.

In addition to the usual sources of noise associated with the spinner magnetometer operation, ${ }^{9}$ unwanted $10-\mathrm{Hz}$ signals may be introduced by the remanent quadrupole moment and anisotropic electrical conductivity of the rotating specimen as well as by second harmonics of various $5-\mathrm{Hz}$ signals. Although these $5-\mathrm{Hz}$ signals may be partly associated with effects of anisotropic susceptibility and conductivity, by far the strongest second harmonic is generated by the remanent dipole moment. Signals produced exclusively by external magnetic field components parallel to the

\footnotetext{
- Noise sources include background electromagnetic fields which may be at least partially cancelled by external shielding or by budking coils, electrostatic charge on the surface of the plastic sample holder which may be effectively removed by commercially available phonograph record sprays or by steam (although the $5-\mathrm{Hz}$ fundamental frequency is removed by filtering, the $10-\mathrm{Hz}$ second harmonic will be passed) mechanical vibrations associated with the rotating shaft or with the pickup coil, and many noise sources associated with electronic components of the pickup and detection system (for a comprehensive review of these latter sources, see Ficchi (1964) and King (1966)). The noise level of the entire system was such that the lower limit of precisely measurable susceptibility difference was approximately $5 \times 10^{-7}$ $\mathrm{emu} / \mathrm{cm}^{3}$, and the lower limit of detectable susceptibility difference was about $5 \times 10^{-3} \mathrm{emu} /$ $\mathrm{cm}^{3}$.
} 
spinner shaft may be eliminated by cancelling these field components by using bucking coils or by orienting the spinner shaft perpendicular to the magnetic meridian of the laboratory. In previous studies of volcanic rocks (Hanna, 1965), the spinner shaft was oriented parallel to the magnetic meridian so that a single horizontally oriented coil or permanent magnet could be used to null all of the horizontal external field component, leaving only a vertical component of external field.

For volcanic rocks, the $10-\mathrm{Hz}$ second harmonic signal associated with the remanent dipole moment is often many times larger than the desired signal associated with susceptibility anisotropy. In order to eliminate the effect of this second harmonic signal, a signal is introduced by the reference circuit component of the spinner magnetometer, ordinarily used for measuring remanent magnetization. This reference circuit component consists of a reference coil made up of two orthogonal square-section coils wound on a plastic cube matching the size of the cubical rock specimen and placed directly over a flat-lying pickup coil identical with the pickup coil directly beneath the specimen holder (fig. 10). An external field produced by the vertically oriented Helmholtz coil is then adjusted until the field surrounding the specimen holder is cancelled. Rotation of the rock specmien in the resulting field-free space produces an output due to the second harmonic of the remanent dipole moment and to the remanent quadrupole moment. For the volcanic rocks studied, the second harmonic signal associated with the dipole moment was always at least an order of magnitude stronger than the signal associated with the quadrupole moment, and therefore the combined signal was almost purely sinusoidal in form. ${ }^{10}$ This sinusoidal output is effectively nulled by introducing the correct proportions of direct current through each of the orthogonal coils of the rotating reference coil, that is, by adjusting the magnitude and phase of the reference circuit signal. After balance is achieved, a vertical external field is introduced within the Helmholtz coil, giving rise to the desired output associated with susceptibility anisotropy and to unwanted output associated with electrical conductivity.

Although there is no provision in the apparatus to eliminate possible signals associated with electrical conductivity, and although no attempt was made to measure the electric conductivity of rock specimens by using other equipment, there is reason to believe that the effects of conductivity were negligibly small for the rock specimens measured. For example, rocks possessing nearly

20 Of 106 specimens studied the remanent quadrupole signal was detectable in only 16 , that is, 15 percent of all measured specimens. 
isotropic susceptibility produced no observable signal, indicating an absence of conductivity effects. Also, rocks possessing anisotropic susceptibility generated an almost purely sinusoidal signal, suggesting that signals associated with conductivity were absent or were fortuitously in-phase or anti-phase with respect to the anisotropic susceptibility signal. Because the amplitudes of signals associated with electrical conductivity are directly proportional not only to the size and conductivity of the conducting material, but also to the time rate of change of the external magnetic field (see, for example, Chikazumi, 1964; Olsen, 1966), the relatively slow rotational speed chosen for the system tends to diminish conductivity effects.

\section{BHATTACHARYA SOLUTION OF EQUATION OF ANISOTROPY}

Having shown in equations 59, 60, and 61 that observed data from spinner magnetometer measurements and one bridge-type measurement directly determine values for $K_{i j}(i, j=1,2,3)$, we proceed to find the principal susceptibility axes by solving the cubic equation represented by equation 44 . Using the terminology of Bhattacharya (1950), we write

$$
\begin{aligned}
& a=K_{11} ; b=K_{22} ; c=K_{s s} \\
& f=K_{2 s} ; g=K_{1 s} ; h=K_{12} .
\end{aligned}
$$

The cubic equation 44 , after expansion of the determinant, is

$$
\begin{aligned}
K^{3}-(a+b+c) K^{2}+ & \left(b c+c a+a b-f^{2}-g^{2}-h^{2}\right) K \\
& -\left(a b c+2 f g h-a f^{2}-b g^{2}-c h^{2}\right)=0 .
\end{aligned}
$$

The three real roots, $K=K_{1}, K_{2}, K_{s}$ may be routinely determined by numerical methods used in digital-computer programming. However, where digital computer access is not available, it is useful to have closed-form analytical expressions for $K_{1}, K_{2}$, and $K_{s}$. Thus, we proceed with the solution of the equation of anisotropy as presented by Bhattacharya $(1950$, appendix 1, p. 94), using standard analytical techniques (see, for example, Dresden, 1930 ; Uspensky, 1948). Setting

$$
K=X+\frac{1}{3}(a+b+c),
$$

equation 66 then becomes

$$
X^{3}-q X-r=0,
$$


where

$$
\begin{aligned}
& q= \frac{1}{3}(a+b+c)^{2}-\left(b c+c a+a b-f^{2}-g^{2}-h^{2}\right) \\
& r=\frac{2}{27}(a+b+c)^{3} \\
&- \\
&-\frac{1}{3}(a+b+c)\left(b c+c a+a b-f^{2}-g^{2}-h^{2}\right) \\
&+\left(a b c+2 f g h-a f^{2}-b g^{2}-c h^{2}\right) .
\end{aligned}
$$

Because equation 68 can be solved trigonometrically, we make the substitution

$$
X=N Y
$$

and, from equation 68 , we write

$$
Y^{3}-\frac{q}{N^{2}} Y-\frac{r}{N^{3}}=0 \text {. }
$$

If we use the trigonometric identity

$$
\cos ^{3} \eta-\frac{3}{4} \cos \eta-\frac{1}{4} \cos 3 \eta=0
$$

and assume

$$
Y=\cos \eta
$$

in equation 72 , we have, by comparing equations 72 and 73 ,

and

$$
N=2 \sqrt{\frac{\bar{q}}{3}}
$$

$$
\cos 3 \eta=\frac{4 r}{N^{3}}=\frac{r}{2} \sqrt{\frac{27}{q^{3}}}
$$

The term, $\cos \eta$, may be determined from equation 76 by trigonometric tabulations, the three roots of equation 72, expressed as

$$
Y=\cos \eta, \cos \left(\eta+\frac{2 \pi}{3}\right), \cos \left(\eta+\frac{4 \pi}{3}\right) \text {. }
$$

From equations 67,71 , and 75 , the three values of susceptibility are

where

$$
K=\frac{1}{3}(a+b+c)+2 \sqrt{\frac{q}{3}} \cos \left(\eta+\frac{S_{\pi}}{3}\right)
$$

$$
S=0,2,4 \text {. }
$$


Because the first term of equation 78 is the bulk susceptibility, as defined by equation 63 or 64 , we may consider each principal susceptibility component to consist of the algebraic sum of the bulk susceptibility of the sample and a term associated with anisotropy. Of further interest is that the sum of equations 78 , with substituted values of $S=0,2$, and 4 , is

$$
\begin{gathered}
K_{1}+K_{2}+K_{s}=a+b+c \\
=K_{11}+K_{22}+K_{s s},
\end{gathered}
$$

an invariant property of the second-rank tensor.

This invariant relationship between the sum of principal susceptibilities and the sum of normal susceptibilities may be used to demonstrate a practical procedure in the calculation of anisotropy. Because values of normal susceptibilities, $K_{11}, K_{22}$, and $K_{s s}$, are ordinarily orders of magnitude larger than values of cross susceptibilities, computation of small percentages of anisotropy requires retention of large numbers of digits. If, however, the measured normal susceptibility, say $K_{s s}=K_{s s}^{*}$, is set equal to zero, corresponding normal susceptibilities have small values, similar to those of the cross susceptibilities, and fewer digits are required for computation. The corresponding computed principal susceptibilities of equation 78 are subsequently increased by addition of the constant, $K_{s s}^{*}$, to obtain the principal susceptibility components. This procedure is evident from equations 62 and 79 , which we combine as follows

$$
\begin{gathered}
\left(K_{11}+K_{22}+K_{s s}\right)_{K s s}=K_{s s}^{*}=A \cos 2 \alpha+2 B \cos 2 \beta+3 K_{s s}^{*} \\
=K_{1}+K_{2}+K_{s} .
\end{gathered}
$$

If $K_{s s}$ is set equal to zero, we have

$$
\begin{gathered}
\left(K_{11}+K_{22}+K_{s s}\right)_{K s s}=0=A \cos 2 \alpha+2 B \cos \beta \\
=\left(K_{1}-K_{s s}^{*}\right)+\left(K_{2}-K_{s s}^{*}\right)+\left(K_{s}-K_{s s}^{*}\right) .
\end{gathered}
$$

The corresponding principal susceptibilities are deficient by the term $K_{s s}^{*}$, and this term must be added to computed values $K$, $K_{2}$, and $K_{s}$. The procedure of setting the measured normal susceptibility equal to zero and subsequently adding this constant to computed principal susceptibilities is most important where computation facilities are insufficient to accommodate large numbers of digits (significant figures).

The direction cosines of each principal axis may be determined by using equation 44 , following the procedure of Bhattacharya 
$(1950$, p. 96). If we rewrite these equations in previously defined coefficients, we have

$$
\begin{aligned}
& a_{o} l_{1}+h l_{2}+g l_{s}=0 \\
& h l_{1}+b_{o} l_{2}+f l_{s}=0 \\
& g l_{1}+f l_{2}+c_{o} l_{s}=0
\end{aligned}
$$

where

$$
\begin{aligned}
& a_{o}=a-K \\
& b_{o}=b-K \\
& c_{o}=c-K .
\end{aligned}
$$

When we eliminate $l_{2}$ between the first two equations of 80 , we have

$$
l_{1}=\left(\frac{b_{o} g-f h}{h^{2}-a_{o} b_{o}}\right) l_{3}
$$

and when we eliminate $l_{1}$ between the second two equations of 80 , we have

$$
l_{2}=\left(\frac{c_{o} h-f g}{b_{o} g-f h}\right) l_{3} .
$$

We may use the ratios, $m$ and $n$, obtained from equations 82 and 83 , and write

$$
\begin{gathered}
m=\frac{l_{1}}{l_{s}}=\frac{b_{o} g-f h}{h^{2}-a_{o} b_{o}} \\
n=\frac{l_{2}}{l_{s}}=\frac{c_{o} h-f g}{b_{o} g-f h}
\end{gathered}
$$

to compute the direction cosines, or, alternatively the inclinations and declinations of the principal susceptibility axes.

If a radius vector, $\vec{R}$, passing through the the origin of the magnitude ellipsoid is taken parallel to a principal susceptibility axis; then the unit vector $\vec{R}_{o}$ along this axis has direction cosines $l_{1}, l_{2}$, and $l_{s}$, such that

$$
\begin{aligned}
& \vec{R}_{o}=l_{1} \vec{X}_{1}+l_{2} \vec{X}_{2}+l_{s} \vec{X}_{s} \\
& =\frac{X_{1}}{R} \vec{X}_{1}+\frac{X_{2}}{R} \vec{X}_{2}+\frac{X_{s}}{R} \vec{X}_{s}
\end{aligned}
$$

where $\vec{X}_{1}, \vec{X}_{2}$, and $\vec{X}_{s}$ are unit vectors along the specimen coordinate axes. From equation 86

$$
R=\frac{X_{1}}{l_{1}}=\frac{X_{2}}{l_{2}}=\frac{X_{3}}{l_{s}},
$$


which is the equation of the line along which the principal axis lies. If we rewrite equation 87 , we have

$$
\frac{X_{1}}{l_{1} / l_{s}}=\frac{X_{2}}{l_{2} / l_{s}}=X_{s}
$$

or, using equations 84 and 85 ,

$$
\frac{X_{1}}{m}=\frac{X_{\varepsilon}}{n}=X_{s}
$$

When we note from equation 89 that

$$
X_{1}=\frac{m}{n} X_{2}
$$

and

$$
X_{s}=\frac{X_{2}}{n}
$$

and if we use relation 87 , we have

$$
\begin{aligned}
& l_{1}=\frac{\frac{m}{n} X_{2}}{R}, \\
& l_{2}=\frac{X_{2}}{R},
\end{aligned}
$$

and

$$
l_{s}=\frac{X_{2} / n}{R}
$$

where

$$
R=\sqrt{\left(\frac{m}{n}\right)^{2} X_{2}^{2}+X_{2}^{2}+\left(\frac{X_{2}}{n}\right)^{2}}=\frac{X_{2}}{n} \sqrt{m^{2}+n^{2}+1}
$$

When we substitute equation 95 into equations 92,93 , and 94, we have for the direction cosines of the principal axis

$$
\begin{aligned}
& l_{1}=\frac{m}{\sqrt{m^{2}+n^{2}+1}}, \\
& l_{2}=\frac{n}{\sqrt{m^{2}+n^{2}+1}},
\end{aligned}
$$

and

$$
l_{s}=\frac{1}{\sqrt{m^{2}+n^{2}+1}}
$$


For ease of plotting, we may express the direction of the principal susceptibility axis in terms of inclination and declination (Bhattacharya, 1950, p. 98). If $I^{\prime}$ is the inclination and $D^{\prime}$ is the declination measured positive from the $+X_{1}$ to the $+X_{2}$ axis (fig. 11),

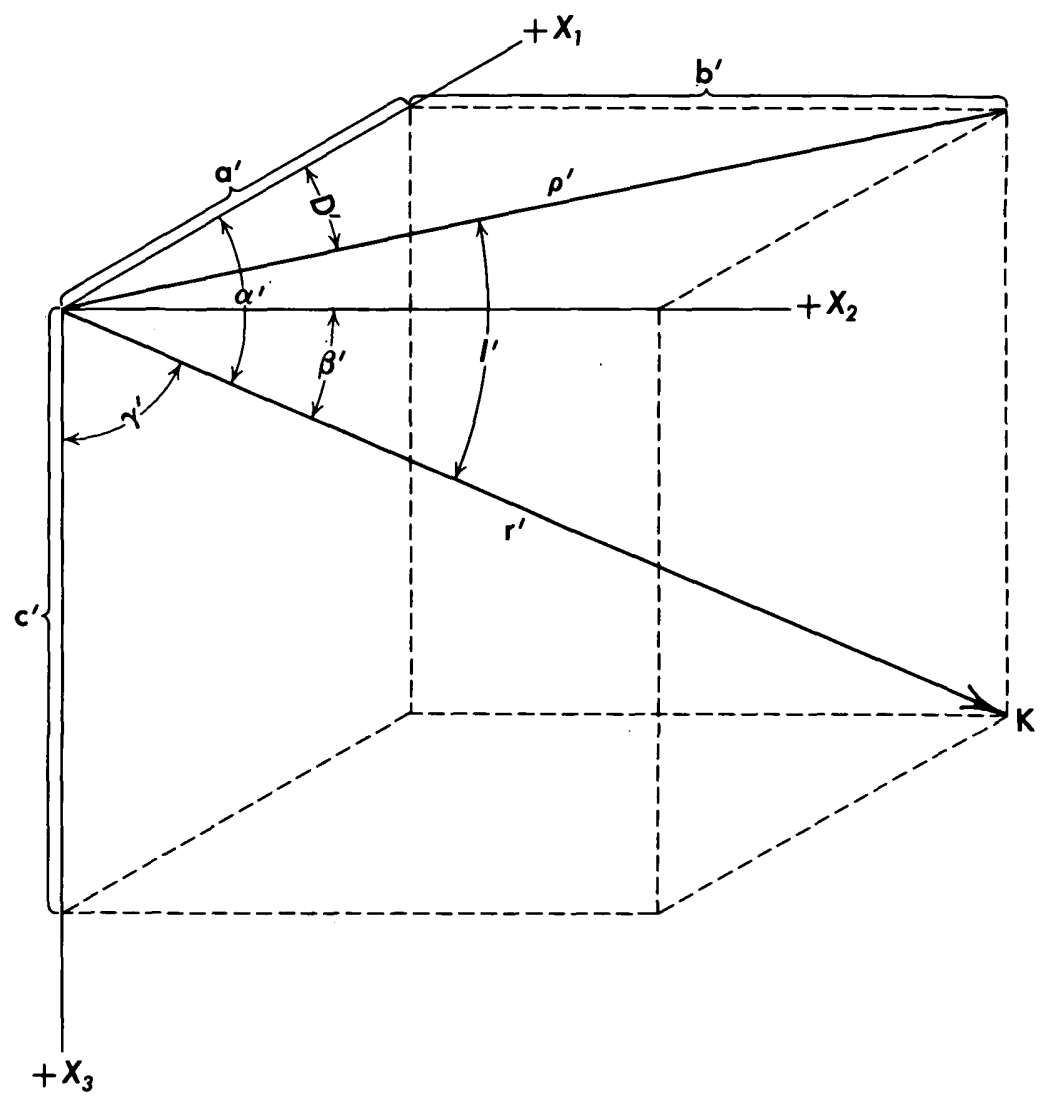

From diagram:

(1) $\cos D^{\prime}=\frac{a^{\prime}}{\rho^{\prime}}$, (2) $\sin D^{\prime}=\frac{b^{\prime}}{\rho^{\prime}}$, (3) $\cos l^{\prime}=\frac{\rho^{\prime}}{r^{\prime}}$, (4) $\sin l^{\prime}=\frac{c^{\prime}}{r^{\prime}}$,

(5) $I_{1}=\cos \alpha^{\prime}=\frac{a^{\prime}}{r^{\prime}}$, (6) $I_{2}=\cos \beta^{\prime}=\frac{b^{\prime}}{r^{\prime}}, \quad$ (7) $I_{3}=\cos \gamma^{\prime}=\frac{c^{\prime}}{r^{\prime}}$,

Therefore:

$$
\begin{array}{cc}
\cos \alpha^{\prime}=\cos D^{\prime} \cos I_{;}^{\prime} & \cos \beta^{\prime}=\sin D^{\prime} \cos I^{\prime} \\
\tan D^{\prime}=\frac{\cos \beta^{\prime}}{\cos \alpha^{\prime}}=\frac{I_{2}}{I_{1}} ; & \sin I^{\prime}=\cos \gamma^{\prime}=I_{3}
\end{array}
$$

FIGURE 11.-Relationship of declination, $D^{\prime}$, and inclination, $I^{\prime}$, to the direction cosines, $l_{1}, l_{2}$, and $l_{3}$ of a principal susceptibility axis referred to the rock sample coordinate system. 
we have

$$
\begin{aligned}
& l_{1}=\cos D^{\prime} \cos I^{\prime}, \\
& l_{2}=\sin D^{\prime} \cos I^{\prime}, \\
& l_{s}=\sin I^{\prime} .
\end{aligned}
$$

If we divide equation 100 by equation 99 and use equation 95 , we have

$$
\begin{gathered}
\tan D^{\prime}=\frac{l_{2}}{l_{1}}=\frac{n}{m} \\
\sin I^{\prime}=\frac{1}{\sqrt{m^{2}+n^{2}+1}} .
\end{gathered}
$$

The direction cosines or inclination and declination of each principal axis may be determined by the above procedures, provided the appropriate principal susceptibility value is substituted into expressions for $a_{o}, b_{o}$, and $c_{o}$, in equations 81 .

Estimates of uncertainties or errors in computed magnitudes and directions (orientations) of principal susceptibilities may be made in various ways, according to available information about errors in the magnetometer data. Standard expressions involving Taylor expansions have been developed for cases in which (1) the actual errors in data are assumed to be known and (2) some characteristic of errors in the data, such as the standard deviation of a set of measurements, is known (Beers, 1957; Parratt, 1961; Bevington, 1969). These expressions give either the error or error characteristic of the computed result by accounting for propagation of errors associated with the data. For purposes of illustration, we may consider the special case in which principal susceptibility axes coincide with coordinate axes of the specimen or specimen holder.

Considering the situation in which errors in measured values of $A$ and $\alpha$, given by $\Delta A$ and $\Delta \alpha$, are assumed to be known from previous experimental work, we may write for the error or uncertainty of the difference of principal susceptibilities, $\Delta \Lambda$,

$$
\begin{gathered}
\Delta \Lambda=\Delta\left(K_{11}-K_{22}\right) \\
=\Delta\left(K_{1}-K_{2}\right) \\
=\left[(\Delta A) \frac{\partial \Lambda}{\partial A}+(\Delta \alpha) \frac{\partial \Lambda}{\Delta \alpha}\right]+\left[(\Delta A)^{2} \frac{\partial^{2} \Lambda}{\partial A^{2}}+2(\Delta A)(\Delta \alpha) \frac{\partial^{2} \Lambda}{\partial A \partial \alpha}+\right. \\
\left.(\Delta \alpha)^{2} \frac{\partial^{2} \Lambda}{\partial \alpha^{2}}\right]+\left[(\Delta A)^{3} \frac{\partial^{3} \Lambda}{\partial A^{3}}+3(\Delta A)^{2}(\Delta \alpha) \frac{\partial^{3} \Lambda}{\partial A^{2} \partial \alpha}\right.
\end{gathered}
$$




$$
\begin{aligned}
& \left.+3(\Delta A)(\Delta \alpha)^{2} \frac{\partial^{3} \Lambda}{\partial A \partial \alpha^{2}}+(\Delta \alpha)^{3} \frac{\partial^{3} \Lambda}{\partial \alpha^{3}}\right]+\left[(\Delta A)^{4} \frac{\partial^{4} \Lambda}{\partial A^{4}}+\right. \\
& 4(\Delta A)^{3}(\Delta \alpha) \frac{\partial^{4} \Lambda}{\partial A^{3} \partial \alpha}+6(\Delta A)^{2}(\Delta \alpha)^{2} \frac{\partial^{4} \Lambda}{\partial A^{2} \partial \alpha^{2}}+4(\Delta A)(\Delta \alpha)^{3} \frac{\partial^{4} \Lambda}{\partial A \partial \alpha^{3}} \\
& \left.+(\Delta \alpha) \frac{{ }^{4}}{\partial \alpha^{4} \Lambda}\right]+\ldots \\
& =[(\Delta A)(\cos 2 \alpha)-2(\Delta a) A \sin 2 a] \\
& +\left[-4(\Delta \alpha)^{2} A \cos 2 \alpha-4(\Delta A)(\Delta \alpha) \sin 2 \alpha\right] \\
& +\left[-12(\Delta A)(\Delta \alpha)^{2} \cos 2 \alpha\right. \\
& \left.+8(\Delta \alpha)^{2} A \sin 2 \alpha\right] \\
& +\left[32(\Delta A)(\Delta \alpha)^{3} \sin 2 \alpha\right. \\
& \left.+16(\Delta \alpha)^{4} A \cos 2 \alpha\right]+\cdots \text {. }
\end{aligned}
$$

If, in our example, $\alpha=0^{\circ}$ (corresponding to principal susceptibility axes coincident with axes of the specimen or specimen holder), the previously determined uncertainty in axis direction is $\pm 2^{\circ}$, the measured susceptibility difference is $10^{-5} \mathrm{emu} / \mathrm{cm}^{3}$, and the previously determined error in this difference is \pm 5 percent, or $\pm 5 \times 10^{-7} \mathrm{emu} / \mathrm{cm}^{3}$ (corresponding to uncertainty in the measurement of electric current), the resulting error in susceptibility difference, $\Delta \Lambda$, ranges from $-5.41 \times 10^{-7}$ to $+4.44 \times 10^{-7}$ $\mathrm{emu} / \mathrm{cm}^{3}$. These figures are typical of propagated errors in the present study.

\section{SAMPLE CALCULATION}

As an example of the calculation of susceptibility anisotropy, data are presented which were obtained from measurements of a 2.54-cm cubical specimen of vesicular basalt from the Elkhorn Mountains Volcanics (Upper Cretaceous) northeast of Whitehall, Mont. The $\mathrm{X}_{1}-\mathrm{X}_{2}$ coordinate plane of the sample, as measured in the field, has a strike of N. $38^{\circ} \mathrm{W}$. and a dip of $77^{\circ} \mathrm{NE}$. The basaltic unit itself has a strike of N. $55^{\circ} \mathrm{W}$. and a dip of $60^{\circ} \mathrm{NE}$., as determined by the planar orientation of vesicles and the orientations of volcanic rock units stratigraphically above and below the basalt. The rock specimen, which was collected near the central part of the 82-m-thick flow unit, has a stable reversed remanent magnetization with declination, N. $54^{\circ}$ E., inclination, $-39^{\circ}$, and intensity, $1.04 \times 10^{-3} \mathrm{emu} / \mathrm{cm}^{3}$.

Apparently susceptibility ${ }^{11}$ magnitudes and associated phase angles obtained by using the Mead-type spinner magnetometer are

\footnotetext{
11 Apparent susceptibility values mav be converted to true susceptibility values using equation 3 and the demagnetization factor, $N=4 \pi / 3$.
} 


$$
\begin{aligned}
& A=2.13 \times 10^{-5} \mathrm{emu} / \mathrm{cm}^{3}, \\
& B=7.81 \times 10^{-5} \mathrm{emu} / \mathrm{cm}^{3} . \\
& C=9.80 \times 10^{-5} \mathrm{emu} / \mathrm{cm}^{3}, \\
& \alpha=173^{\circ}, \\
& \beta=7^{\circ}, \\
& \gamma=85^{\circ},
\end{aligned}
$$

and for the independent measurement of $\mathrm{K}_{3: 3}$,

$$
K_{s s}^{*}=2.30 \times 10^{-3} \mathrm{emu} / \mathrm{cm}^{3} \text {. }
$$

In the computation process, we initially set $K_{33}=0$ so that, from equations 59,60 , and 65 , we write to three significant figures,

$$
\begin{aligned}
& a=9.51 \times 10^{-5} \mathrm{emu} / \mathrm{cm}^{3} \\
& b=7.58 \times 10^{-5} \mathrm{emu} / \mathrm{cm}^{3} \\
& c=0 \\
& f=0.945 \times 10^{-5} \mathrm{emu} / \mathrm{cm}^{3} \\
& g=0.851 \times 10^{-5} \mathrm{emu} / \mathrm{cm}^{3}
\end{aligned}
$$

and

$$
h=-0.258 \times 10^{-5} \mathrm{emu} / \mathrm{cm}^{3} .
$$

Also, from equations 66 through 78 , we have

$1 / 3(a+b+c)=5.70 \times 10^{-5} \mathrm{emu} / \mathrm{cm}^{3}$

$1 / 3(a+b+c)^{2}=97.3 \times 10^{-5}$ units,

$2 / 27(a+b+c)^{3}=370 \times 10^{-5}$ units,

$$
\begin{aligned}
& b c+a c+a b-f^{2}-g^{2}-h^{2}=70.4 \times 10^{-5} \text { units, } \\
& a b c+2 f g h-a f^{2}-b g^{2}-c h^{2}=-14.4 \times 10^{-5} \text { units, } \\
& q=26.9 \times 10^{-5} \text { units, } \\
& r=-45.7 \times 10^{-5} \text { units, } \\
& \cos 3 \eta=-0.849 \\
& 3 \eta=148^{\circ} \\
& \eta=49.4^{\circ} \\
& \cos \eta=0.651 \\
& \cos \left(\begin{array}{rl}
\eta \pi \\
3
\end{array}\right)=-0.983 \\
& \cos \left(\begin{array}{rl}
2 \pi \\
\eta
\end{array}\right)=0.331 \\
& N=5.99 \times 10^{-5} \mathrm{units}, \\
& K_{1}=9.60 \times 10^{-5} \mathrm{emu} / \mathrm{cm}^{3} \\
& K_{2}=-0.195 \times 10^{-5} \mathrm{emu} / \mathrm{cm}^{3}
\end{aligned}
$$


and

$$
K_{s}=7.68 \times 10^{-5} \mathrm{emu} / \mathrm{cm}^{3} .
$$

To find the actual values of principal susceptibilities, we must add the term $K_{s s}^{*}=2.30 \times 10^{-3} \mathrm{emu} / \mathrm{cm}^{3}$ to each of the above terms, $K_{1} K_{2}, K_{s}$. The actual principal susceptibility values, $K_{1}^{*} K_{2}^{*}$ $K_{s}^{*}$, are

$$
\begin{aligned}
& K_{1}^{*}=2.30 \times 10^{-3}+9.60 \times 10^{-5}=2.40 \times 10^{-3} \mathrm{emu} / \mathrm{cm}^{3}, \\
& K_{2}^{*}=2.30 \times 10^{-3}-0.195 \times 10^{-5}=2.30 \times 10^{-3} \mathrm{emu} / \mathrm{cm}^{3},
\end{aligned}
$$

and

$$
K_{s}^{*}=2.30 \times 10^{-3}+7.68 \times 10^{-5}=2.38 \times 10^{-3} \mathrm{emu} / \mathrm{cm}^{3} .
$$

The magnitude ellipsoid has the shape of an oblate spheroid with the maximum plane of susceptibility normal to $K_{2}^{*}$, the minor axis. The maximum percent anisotropy, as defined by equation 74, is

Maximum percent anisotropy $=\left(\frac{9.80 \times 10^{-5}}{2.36 \times 10^{-3}}\right) 100$ percent

$$
=4.15 \text { percent. }
$$

The direction of the $K_{1}$ principal susceptibility axis is obtained from equations 81 through 103, as follows:

$$
\begin{aligned}
a_{o} & =-0.0911, \\
b_{o} & =-2.02, \\
c_{o} & =-9.60, \\
m & =12.5, \\
n & =0.379,
\end{aligned}
$$

with direction cosines $l_{1}, l_{2}$, and $l_{3}$,

$$
\begin{aligned}
& l_{1}=0.996 \\
& l_{2}=0.0301 \\
& l_{s}=0.0795,
\end{aligned}
$$

and their associated angles $\theta_{1}, \theta_{2}$, and $\theta_{3}$,

$$
\begin{aligned}
& \theta_{1}=4.88^{\circ}, \\
& \theta_{2}=88.2^{\circ},
\end{aligned}
$$

and

$$
\theta_{s}=85.4^{\circ} \text {. }
$$

The declination, $D^{\prime}$, and inclination, $I^{\prime}$, are obtained from

$$
\begin{aligned}
\tan D^{\prime} & =0.0303, \\
D^{\prime} & =1.73^{\circ} \\
\sin I^{\prime} & =0.0795,
\end{aligned}
$$


and

$$
I^{\prime}=4.56^{\circ} \text {. }
$$

The direction of the $K_{2}$ principal susceptibility axis is obtained from equivalent values, as follows :

$$
\begin{aligned}
a_{o} & =9.70, \\
b_{o} & =7.77, \\
c_{o} & =-0.195, \\
m & =-0.091, \\
n & =-0.110, \\
l_{1} & =-0.090, \\
l_{2} & =-0.109, \\
l_{s} & =0.990, \\
\theta_{1} & =95.2^{\circ}, \\
\theta_{2} & =96.2^{\circ}, \\
\theta_{s} & =8.12^{\circ}, \\
\tan D^{\prime} & =1.21, \\
D^{\prime} & =50.4^{\circ}, \\
\sin I^{\prime} & =0.990, \\
I^{\prime} & =81.9^{\circ} .
\end{aligned}
$$

Similarly, the direction of the $\mathrm{K}_{3}$ principal axis is given by

$$
\begin{aligned}
a_{o} & =1.83, \\
b_{o} & =-0.104, \\
c_{o} & =-7.68, \\
m & =0.604, \\
n & =7.59, \\
l_{1} & =0.0787, \\
l_{2} & =0.988, \\
l_{s} & =0.130, \\
\theta_{1} & =85.5^{\circ}, \\
\theta_{2} & =8.75^{\circ}, \\
\theta_{s} & =82.5^{\circ}, \\
\tan D^{\prime} & =1.26, \\
D^{\prime} & =51.5^{\circ}, \\
\sin I^{\prime} & =0.130,
\end{aligned}
$$

and

$$
I^{\prime}=7.48^{\circ} \text {. }
$$

Projections of the maximum plane of susceptibility and the minor axis, $\mathrm{K}_{2}$, are shown in figure 12 relative to the $X_{1}-X_{2}$ coordinate 
plane of the rock specimen, to geographic coordinates in the original outcrop position, and to the bedding plane. The direction of remanent magnetization, also shown for each of the three orientations in figure 12 is seen to be unrelated to the geometrical configuration of the susceptibility anisotropy.

\section{EXAMPLES OF ANISOTROPY DATA}

Additional examples of results from using the Mead-type spinner apparatus-data on 42 volcanic rock specimens possessing anisotropic susceptibility-are listed in table 2. Most of
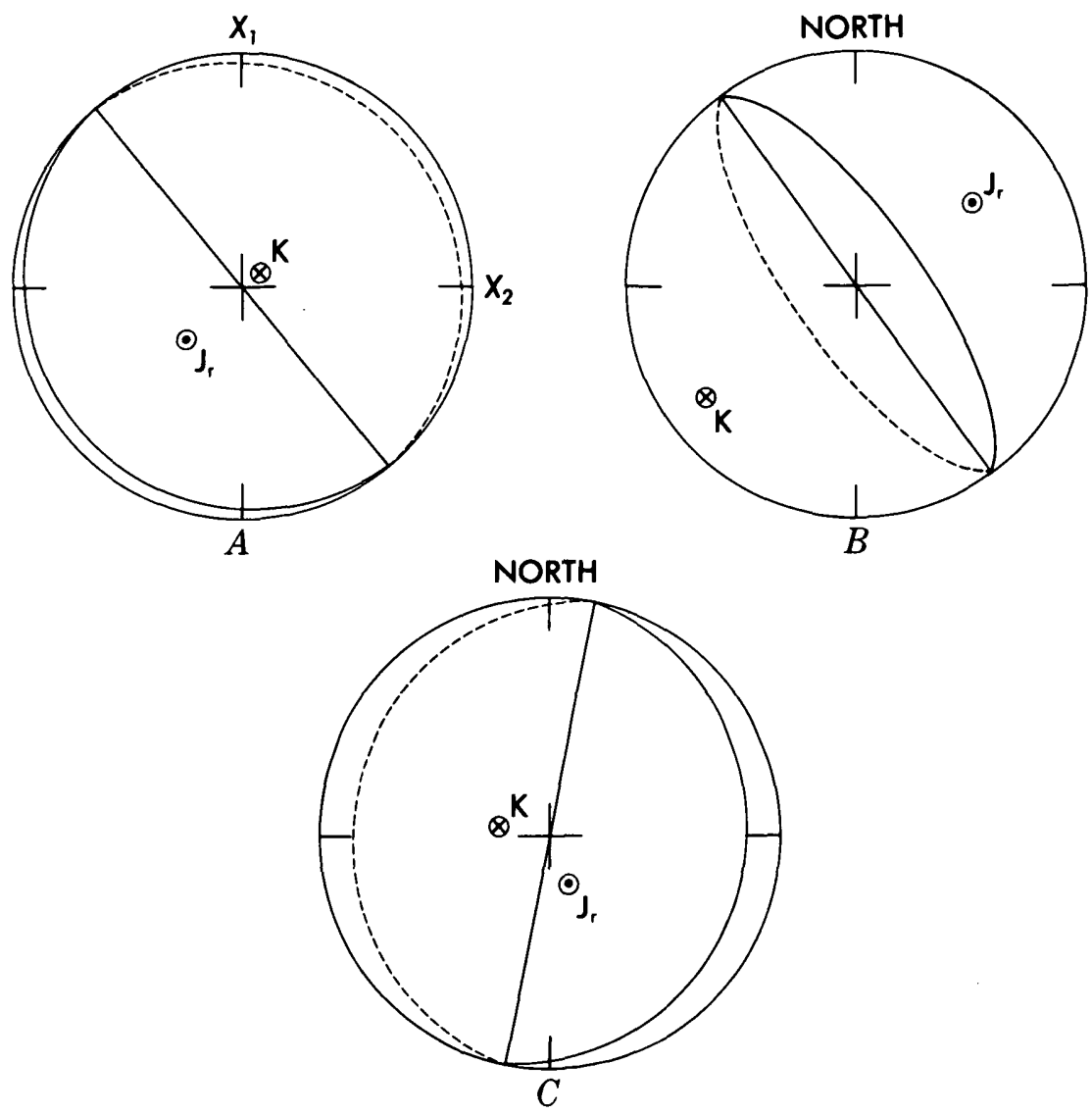

Figure 12.-Plots of maximum susceptibility plane, minor susceptibility axis, and remanent magnetization vector of rock sample relative to $(A)$ the $X_{1}-$ $X_{2}$ coordinate plane of the sample, $(B)$ geographic coordinates in the original outcrop position, and $(C)$ the bedding plane. Solid lines and plots of the minor axis are on the lower hemisphere. Dashed lines and plots of the reversed remanent magnetization vector are on the upper hemisphere. Equalarea projections. 
these samples have relatively strong remanent magnetization intensities of $10^{-4}$ to $10^{-2} \mathrm{emu} / \mathrm{cm}^{3}$, but for all measurements, fundamental frequency signals associated with remanent quadrupole moments were undetected, and second-harmonic signals associated with remanent dipole moments were effectively cancelled. These 42 specimens are part of a collection of 106 samples tested for anisotropy from among 77 lithologic units in the Elkhorn Mountains Volcanics (Upper Cretaceous) of western Montana. Among the 64 specimens not listed in table 2, approximately two-thirds have anisotropies which are detectable but not precisely measurable; the remaining one-third have no detectable anisotropy. Because of the reconnaissance nature of the sampling, no firm conclusions about the relationships between anisotropy configuration and rock type or rock fabric can be confidently drawn. In general, most of these rocks have anisotropies which are triaxial ellipsoidal or nonellipsoidal, although those that possess oblate spheroidal anisotropy have maximum susceptibility planes which are oriented nearly parallel to bedding planes or planes of flow.

Available data from susceptibility anisotropy studies of other volcanic and igneous rocks (Girdler, 1961b; Khan, 1962; Stone, 1963; Wing-Fatt and Stacey, 1966; Janák, 1972) suggest that relationships between susceptibility geometry and petrofabric elements of igneous rocks are not as well defined as these relationships in many sedimentary and highly foliated metamorphic rocks.

Intrinsic susceptibility components of the rock may be obtained directly from the measured apparent susceptibility components by use of the rock specimen demagnetizing factor $\mathrm{N}=$ $4 \pi / 3$. The principal intrinsic susceptibilities, $K_{I R 1}, K_{I R 2}$, and $K_{I R s}$ become

$$
\begin{aligned}
& K_{I R 1}=\frac{2.40 \times 10^{-3}}{1-(4.19)\left(2.40 \times 10^{-3}\right)}=2.42 \times 10^{-3} \mathrm{emu} / \mathrm{cm}^{3}, \\
& K_{I R 2}=\frac{2.30 \times 10^{-3}}{1-(4.19)\left(2.30 \times 10^{-3}\right)}=2.32 \times 10^{-3} \mathrm{emu} / \mathrm{cm}^{3}, \\
& K_{I R s}=\frac{2.38 \times 10^{-3}}{1-(4.19)\left(2.38 \times 10^{-3}\right)}=2.40 \times 10^{-3} \mathrm{emu} / \mathrm{cm}^{3} .
\end{aligned}
$$

The intrinsic magnetic susceptibilities of the magnetic mineral grains within the rock specimen may be estimated using the measured volume percent of magnetite, 2.7 percent $^{12}$ and an estimated average mineral grain demagnetization factor of $3.6 \mathrm{ob}-$ tained from various rock magnetic investigations noted by Nagata

\footnotetext{
12 Measured by point counts of one thin section and one polished section.
} 
TABLE 2.-Anisotropic susceptibility data for 42 volcanic rock [nd not determined, because of inconsistency of data. All susceptibilities are apparent

\begin{tabular}{|c|c|c|c|c|c|c|}
\hline Rock type & ${ }^{1} A$ & ${ }^{1} B$ & ${ }^{1} \mathrm{C}$ & ${ }^{2} a$ & ${ }^{2} \beta$ & ${ }^{2} \gamma$ \\
\hline 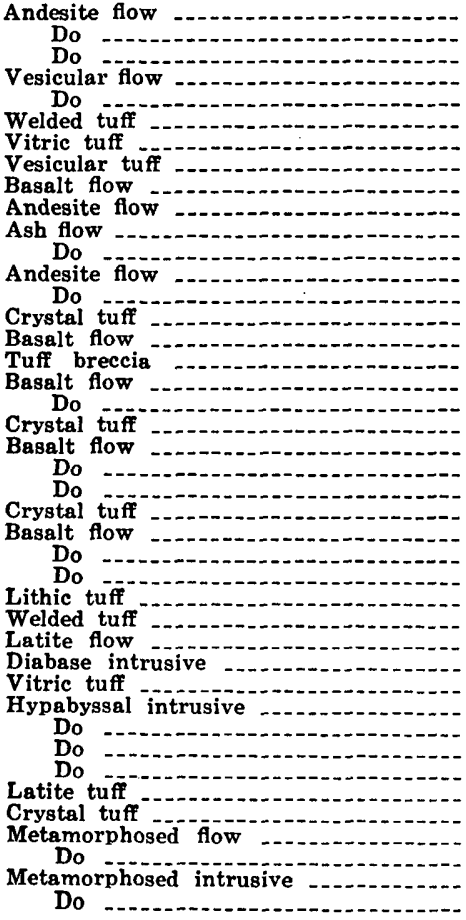 & $\begin{array}{c}33.0 \\
4.04 \\
.55 \\
87.2 \\
82.1 \\
66.6 \\
2.89 \\
21.3 \\
23.6 \\
37.9 \\
49.6 \\
9.12 \\
22.5 \\
13.9 \\
.12 \\
104 \\
19.9 \\
47.3 \\
281 \\
124 \\
109 \\
179 \\
22.3 \\
124 \\
129 \\
4.96 \\
11.1 \\
25.0 \\
43.0 \\
20.1 \\
310 \\
61.6 \\
8.00 \\
67.4 \\
48.0 \\
307 \\
45.7 \\
17.9 \\
1,190 \\
105 \\
744 \\
55.1\end{array}$ & $\begin{array}{c}14.8 \\
2.02 \\
.48 \\
47.7 \\
281 \\
2.40 \\
14.2 \\
78.1 \\
54.4 \\
29.1 \\
10.1 \\
4.05 \\
1.34 \\
5.02 \\
7.30 \\
115 \\
134 \\
14.0 \\
199 \\
79.6 \\
173 \\
159 \\
5.71 \\
150 \\
.50 \\
2.92 \\
26.9 \\
12.4 \\
16.6 \\
.12 \\
260 \\
17.6 \\
17.9 \\
.88 \\
76.1 \\
333 \\
7.64 \\
3.62 \\
626 \\
81.6 \\
320 \\
104\end{array}$ & $\begin{array}{c}4.34 \\
10.7 \\
.99 \\
74.6 \\
49.9 \\
6.31 \\
.06 \\
98.0 \\
28.3 \\
40.1 \\
54.3 \\
16.6 \\
2.32 \\
7.40 \\
7.82 \\
135 \\
39.9 \\
13.5 \\
27.0 \\
15.8 \\
112 \\
161 \\
5.42 \\
94.1 \\
19.1 \\
17.1 \\
9.90 \\
.48 \\
49.8 \\
19.1 \\
17.0 \\
61.6 \\
13.1 \\
317 \\
120 \\
309 \\
19.4 \\
13.7 \\
1,030 \\
547 \\
417 \\
54.9\end{array}$ & $\begin{array}{r}150 \\
106 \\
78 \\
75 \\
22 \\
48 \\
8 \\
173 \\
56 \\
32 \\
6 \\
89 \\
42 \\
16 \\
55 \\
154 \\
75 \\
148 \\
106 \\
156 \\
47 \\
34 \\
146 \\
17 \\
37 \\
82 \\
81 \\
39 \\
70 \\
101 \\
179 \\
73 \\
86 \\
73 \\
36 \\
72 \\
60 \\
83 \\
14 \\
164 \\
174 \\
68\end{array}$ & $\begin{array}{r}77 \\
66 \\
95 \\
158 \\
24 \\
10 \\
52 \\
7 \\
37 \\
29 \\
156 \\
69 \\
89 \\
106 \\
172 \\
153 \\
88 \\
119 \\
119 \\
86 \\
73 \\
175 \\
118 \\
55 \\
55 \\
71 \\
68 \\
60 \\
103 \\
5 \\
174 \\
50 \\
81 \\
5 \\
160 \\
135 \\
30 \\
3 \\
134 \\
170 \\
89 \\
91\end{array}$ & $\begin{array}{r}74 \\
32 \\
5 \\
154 \\
163 \\
21 \\
55 \\
85 \\
53 \\
26 \\
91 \\
22 \\
58 \\
85 \\
105 \\
76 \\
55 \\
103 \\
117 \\
54 \\
78 \\
19 \\
75 \\
116 \\
150 \\
33 \\
56 \\
5 \\
7 \\
7 \\
10 \\
165 \\
109 \\
140 \\
156 \\
169 \\
173 \\
1 \\
79 \\
126 \\
85 \\
84\end{array}$ \\
\hline
\end{tabular}

I A, B, C, magnitudes of anisotropic susceptibility signals in the first, second, and third spin orientations, respectively, adjusted to susceptibility units of $10^{-6} \mathrm{emu} / \mathrm{cm}^{3}$.

$2 a, \beta, \gamma$, phase angles measured in the first, second, and third spin orientations, respeatively, in degrees.

${ }^{3} \mathrm{~K}_{33} \cdot$ normal susceptibility component measured by static method parallel to $\mathrm{X}_{3}$ rock sample coordinate axis, in units of $10^{-6} \mathrm{emu} / \mathrm{cm}^{3}$.

and Uyeda (1961, p. 131). The estimated intrinsic susceptibilities, $K_{I M 1}, K_{I M 2}$, and $K_{I M s}$ are

$$
\begin{aligned}
& K_{I M 1}=\frac{2.42 \times 10^{-3}}{0.027-(3.6)\left(2.42 \times 10^{-3}\right)}=1.32 \times 10^{-1} \mathrm{emu} / \mathrm{cm}^{3}, \\
& K_{I M 2}=\frac{2.32 \times 10^{-3}}{0.027-(3.6)\left(2.32 \times 10^{-3}\right)}=1.24 \times 10^{-1} \mathrm{emu} / \mathrm{cm}^{3},
\end{aligned}
$$


specimens obtained by the dynamic method of measurement

susceptibilities which may be converted to true rock susceptibilities using the rock sample factor of $4 \pi / 3$ ]

\begin{tabular}{|c|c|c|c|c|c|c|}
\hline${ }^{3} K s s$ & ${ }^{\prime} K_{H}$ & ${ }^{8} K_{1}-K_{B}$ & ${ }^{5} K_{2}-K_{B}$ & ${ }^{5} K_{s}-K_{B}$ & $\begin{array}{l}\text { Maxi- } \\
\text { mum } \\
\text { per- } \\
\text { cent } \\
\text { aniso- } \\
\text { tropy }\end{array}$ & $\begin{array}{c}\text { Description } \\
\text { of anisotropy }\end{array}$ \\
\hline $\begin{array}{r}2,450 \\
758 \\
760 \\
2,290 \\
2,310 \\
2,990 \\
639 \\
2,300 \\
3.390 \\
3,010 \\
3,410 \\
3,000 \\
401 \\
3,400 \\
1,610 \\
10,100 \\
4,490 \\
5,910 \\
5.890 \\
5,210 \\
4,990 \\
2,810 \\
2,800 \\
3,190 \\
9,110 \\
509 \\
930 \\
3,490 \\
4,810 \\
1,590 \\
10,100 \\
6,790 \\
620 \\
6,210 \\
2,790 \\
10,000 \\
2,800 \\
1,490 \\
51,100 \\
41,900 \\
50,500 \\
2,540\end{array}$ & $\begin{array}{c}2,447 \\
756.0 \\
759.5 \\
2,284 \\
\text { nd } \\
2,989 \\
638.0 \\
2,357 \\
3,398 \\
\text { nd } \\
3,430 \\
2,995 \\
400.9 \\
3,401 \\
1,614 \\
10,158 \\
\text { nd } \\
5,911 \\
\text { nd } \\
5,186 \\
\text { nd } \\
\text { nd } \\
2,800 \\
3,194 \\
\text { nd } \\
506.0 \\
\text { nd } \\
3,488 \\
4,792 \\
1,584 \\
\text { nd } \\
6,771 \\
\text { nd } \\
6,192 \\
\text { nd } \\
9,892 \\
2,795 \\
1,487 \\
51,390 \\
41,980 \\
50,530 \\
\text { nd }\end{array}$ & $\begin{array}{c}15.0 \\
5.00 \\
.493 \\
55.6 \\
\text { nd } \\
33.0 \\
6.67 \\
39.0 \\
35.4 \\
\text { nd } \\
34.8 \\
7.62 \\
11.3 \\
7.42 \\
3.07 \\
98.1 \\
\text { nd } \\
24.3 \\
\text { nd } \\
50.1 \\
\text { nd } \\
\text { nd } \\
.118 \\
71.6 \\
\text { nd } \\
8.30 \\
\text { nd } \\
12.7 \\
22.1 \\
7.21 \\
\text { nd } \\
26.2 \\
\text { nd } \\
150 \\
\text { nd } \\
279 \\
20.5 \\
7.26 \\
751 \\
276 \\
389 \\
\text { nd }\end{array}$ & $\begin{array}{c}-18.7 \\
-5.98 \\
-.525 \\
-56.4 \\
\text { nd } \\
-33.7 \\
-7.58 \\
-58.9 \\
-27.9 \\
\text { nd } \\
-22.2 \\
-9.36 \\
-11.4 \\
-6.75 \\
-5.28 \\
-88.2 \\
\text { nd } \\
-24.8 \\
\text { nd } \\
-75.1 \\
\text { nd } \\
\text { nd } \\
.116 \\
-116 \\
\text { nd } \\
-8.95 \\
\text { nd } \\
\text {-14.7 } \\
-32.9 \\
-13.4 \\
\text { nd } \\
-44.8 \\
\text { nd } \\
-169 \\
\text { nd } \\
\text {-208 } \\
\text {-25.8 } \\
\text {-10.6 } \\
\text {-728 } \\
-274 \\
-359 \\
\text { nd }\end{array}$ & $\begin{array}{c}3.62 \\
.98 \\
.033 \\
.747 \\
\text { nd } \\
.738 \\
.905 \\
19.8 \\
-7.42 \\
\text { nd } \\
-12.6 \\
1.75 \\
.083 \\
-.673 \\
2.21 \\
-9.97 \\
\text { nd } \\
.473 \\
\text { nd } \\
25.0 \\
\text { nd } \\
\text { nd } \\
-.234 \\
44.8 \\
\text { nd } \\
.650 \\
\text { nd } \\
-1.92 \\
10.8 \\
6.16 \\
\text { nd } \\
18.6 \\
\text { nd } \\
18.9 \\
\text { nd } \\
-71.7 \\
5.21 \\
3.37 \\
-22.3 \\
-2.07 \\
-30.4 \\
\text { nd }\end{array}$ & $\begin{array}{r}1.38 \\
1.45 \\
.13 \\
4.90 \\
\text { nd } \\
2.23 \\
2.23 \\
4.15 \\
1.86 \\
\text { nd } \\
1.66 \\
.57 \\
5.66 \\
.42 \\
.52 \\
1.83 \\
\text { nd } \\
.83 \\
\text { nd } \\
2.41 \\
\text { nd } \\
\text { nd } \\
.01 \\
5.87 \\
\text { nd } \\
3.41 \\
\text { nd } \\
.78 \\
1.15 \\
1.30 \\
\text { nd } \\
1.05 \\
\text { nd } \\
5.14 \\
\text { nd } \\
4.87 \\
1.66 \\
1.20 \\
2.88 \\
1.31 \\
1.48 \\
\text { nd }\end{array}$ & $\begin{array}{l}\text { Triaxial ellipsoid. } \\
\text { Do. } \\
\text { Do. } \\
\text { Do. } \\
\text { Nonellipsoidal. } \\
\text { Triaxial ellipsoid. } \\
\text { Do. } \\
\text { Oblate spheroid. } \\
\text { Triaxial ellipsoid. } \\
\text { Nonellipsoidal. } \\
\text { Prolate spheroid. } \\
\text { Triaxial ellipsoid. } \\
\text { Do. } \\
\text { Do. } \\
\text { Oblate spheroid. } \\
\text { Triaxial ellipsoid. } \\
\text { Nonellipsoidal. } \\
\text { Triaxial ellipsoid. } \\
\text { Nonellipsoidal. } \\
\text { Oblate spheroid. } \\
\text { Nonellipsoidal. } \\
\text { Do. } \\
\text { Oblate spheroid. } \\
\text { Do. } \\
\text { Nonellipsoidal. } \\
\text { Triaxial ellipsoid. } \\
\text { Nonellipsoidal. } \\
\text { Triaxial ellipsoid. } \\
\text { Oblate spheroid. } \\
\text { Do. } \\
\text { Nonellipsoidal. } \\
\text { Oblate spheroid. } \\
\text { Nonellipsoidal. } \\
\text { Triaxial ellipsoid. } \\
\text { Nonellipsoidal. } \\
\text { Triaxial ellipsoid. } \\
\text { Do. } \\
\text { Oblate spheroid. } \\
\text { Triaxial ellipsoid. } \\
\text { Do. } \\
\text { Do. } \\
\text { Nonellipsoidal. }\end{array}$ \\
\hline
\end{tabular}

$K_{B}$ bulk susceptibility, defined as $1 / 3\left(K_{11}+K_{22}+K_{s s}\right)=1 / 3\left(K_{1}+K_{8}+K_{s}\right)$, in units of $10^{-b}$ $\mathrm{emu} / \mathrm{cm}^{3}$.

o $\left.K_{1}-K_{B}\right),\left(K_{2}-K_{B}\right),\left(K_{s}-B\right)$, differences of principal susceptibility components and bulk susceptibility in units of $10^{-6} \mathrm{emu} / \mathrm{cm}^{3}$.

- Maximum percent anisotropy, defined as $\left[\frac{\left(K_{\max }-K_{m i n}\right)}{K_{B}}\right] \times 100$ percent.

$$
K_{I M S}=\frac{2.38 \times 10^{-3}}{0.027-(3.6)\left(2.38 \times 10^{-3}\right)}=1.29 \times 10^{-1} \mathrm{emu} / \mathrm{cm}^{3} \text {. }
$$

We may take as the estimated mean intrinsic susceptibility, $K_{I N I}$, of the magnetic mineral grains the arithmetic mean of $K_{I I I}$, $K_{I M r 2}$, and $K_{I M s}$, which is

$$
K_{I M}=1 / 3\left(K_{I 1}+K_{I 2}+{ }_{I s}\right)=1.28 \times 10^{-1} \mathrm{emu} / \mathrm{cm}^{3} .
$$




\section{REFERENCES CITED}

Balsley, J. R., and Buddington, A. F., 1960, Magnetic susceptibility anisotropy and fabric of some Adirondack granites and orthogneisses: Am. Jour. Sci., v. 258-A (Bradley Volume), p. 6-20.

Bates, L. F., 1961, Modern magnetism: London, Cambridge Univ. Press, 514 p.

Beers, Yardley, 1957, Introduction to the theory of error: Cambridge, Mass., Addison-Wesley Publishing Co., 66 p.

Bevington, P. R., 1969, Data reduction and error analysis for the physical sciences: New York, McGraw-Hill Book Co., 336 p.

Bhagavantam, S., 1966, Crystal symmetry and physical properties: London, Academic Press, $230 \mathrm{p}$.

Bhattacharya, P. K., 1950, An investigation of changes in the magnetic field of the earth (Magnetic anisotropy of sedimentary rocks): Pasadena, Calif., California Inst. Technology, Ph.D. thesis, $100 \mathrm{p}$.

Billings, A. R., 1969, Tensor properties of materials-generalized compliance and conductivity: New York, Interscience Publishers, $171 \mathrm{p}$.

Birss, R. R., 1964, Symmetry and magnetism: Amsterdam, North-Holland Publishing Co.; New York, Interscience Publishers, 252 p.

Bitter, Francis, 1937, Introduction to ferromagnetism: New Yark, McGrawHill Book Co., 314 p.

Bloembergen, N., 1965, Nonlinear optics: New York, W. A. Benjamin, 222 p.

Borisenko, A. I., and Tarapov, I. E., 1968, Vector and tensor analysis with applications: Englewood Cliffs, N.J., Prentice-Hall, 257 p.

Bozorth, R. M., and Chapin, D. M., 1942, Demagnetization factors of rods: Jour. Appl. Physics, v. 13, p. 320-326.

Chikazumi, Soshin, 1964, Physics of magnetism: New York, John Wiley \& Sons, $554 \mathrm{p}$.

Coe, R. S., 1966, Analysis of magnetic shape anisotropy using second-rank tensors: Jour. Geophys. Research, v. 71 , no. 10, p. 2637-2644.

Collinson, D. W., and Creer, K. M., 1960, Measurements in paleomagnetism, in Runcorn, S. K., ed., Methods and techniques in geophysics: New York, Interscience Publishers, v. 1, p. 168-210.

Collinson, D. W., Creer, K. M., and Runcorn, S. K., eds., 1967, Methods in paleomagnetism: New York, Elsevier Publishing Co., 609 p.

Cornelius, P., 1961, Electrical theory on the Giorgi system: London, CleaverHume Press, $187 \mathrm{p}$.

Doell, R. R., and Cox, Allan, 1965, Measurement of the remanent magnetization of igneous rocks: U. S. Geol. Survey Bull. 1203-A, 32 p.

Dresden, Arnold, 1930, Solid analytic geometry and determinants: New York, John Wiley and Sons, $310 \mathrm{p}$.

Dresner, Stephen, 1971, Units of measurement-An encyclopaedic dictionary of units, both scientific and popular, and the quantities they measure: New York, Hastings House, 287 p.

Ficchi, R. F., 1964, Electrical interference: New York, Hayden Book Co., $262 \mathrm{p}$.

Girdler, R. W., 1961a, The measurement and computation of anisotropy of magnetic susceptibility of rocks: Royal Astron. Soc. Geophys. Jour. v. 5, p. 34-44.

— 1961b, Some preliminary measurements of anisotropy of magnetic susceptibility of rocks: Royal Astron. Soc. Geophys. Jour., v. 5, p. 197206. 
Graham, J. W., 1954, Magnetic susceptibility anisotropy, an unexploited petrofabric element [abs.]: Geol. Soc. America Bull., v. 65, no. 12, pt. 2, p. 1257-1258.

1967, Preliminary account of a refined technique for magnetic susceptibility anisotropy measurement of rocks, in Collinson, D. W., Creer, K. M., and Runcorn, S. K., eds., Methods in paleomagnetism: New York, Elsevier Publishing Co., p. 409-424.

Granar, Lars, 1959, Magnetic measurements on Swedish varved sediments: Arkiv. Geofysik, v. 3, no. 1, p. 1-40.

Hamilton, N., and Rees, A. I., 1965, The anisotropy of magnetic susceptibility of the Franciscan rocks of the Diablo Range, central California: California Univ., Scripps Inst. Oceanography, Marine Phys. Lab., MPL Tech. Memo. 164, 38 p.

Hamming, R. W., 1962, Numerical methods for scientists and engineers: New York, McGraw-Hill Book Co., 411 p.

Hanna, W. F., 1965, Magnetic properties of selected volcanic rocks of southwestern Montana: Bloomington, Ind., Indiana Univ., Ph.D. thesis, 207 p.

Hanna, W. F., and Mead, Judson, 1968, Measuring anisotropic magnetic susceptibility of a rotating rock specimen [abs] : Am. Geophys. Union Trans., v. 49 , no. 4 , p. 672 .

Hollingsworth, C. A., 1967, Vectors, matrices, and group theory for scientists and engineers: New York, McGraw-Hill Book Co., 355 p.

Howell, L. G., Martinez, J. D., and Statham, E. H., 1958, Some observations on rock magnetism: Geophysics, v. 23, no. 2, p. 285-298.

Ising, Gustaf, 1943, On the magnetic properties of varved clay: Arkiv. Matematik, Astronomi, Fysik, v. 29A, no. 5, p. 1-37.

Jahren, C. E., 1963, Magnetic susceptibility of bedded iron-formation: Geophysics, v. 28 , no. 5 , p. $756-766$.

Janák, F., 1972, Magnetic susceptibility anisotropy of various rock types and its significance for geophysics and geology: Geophys. Prospecting [Netherlands], v. 20, p. 375-384.

Kaplan, Wilfred, 1952, Advanced calculus: Cambridge, Mass., Addison-Wesley Press, $679 \mathrm{p}$.

Kennelly, A. E., 1936, Magnetic formulae expressed in the M.K.S. system of units: Am. Phil Soc. Proc., v. 76, no. 3, p. 343-377.

Khan, M. A., 1962, The anisotropy of magnetic susceptibility of some igneous and metamorphic rocks: Jour. Geophys. Research, v. 67 , no. 7, p. 28732885.

King, R. A., 1966, Electrical noise: London, Chapman and Hall, 195 p.

King, R. F., 1967, Errors in anisotropy measurements with the torsion balance, in Collinison, D. W., Creer, K. M., and Runcorn, S. K., eds., Methods in paleomagnetism: New York, Elsevier Publishing Co., p. 387-398.

King, R. F., and Rees, A. I., 1962, The measurement of the anisotropy of magnetic susceptibility of rocks by the torque method: Jour. Geophys. Research, v. 67, p. $1565-1572$.

Kittel, Charles, 1953, Introduction to solid state physics: New York, John Wiley \& Sons, $396 \mathrm{p}$.

McGreevy, T., 1953, The M.K.S. system of units: London, Sir Isaac Pitman \& Sons, $283 \mathrm{p}$.

Maxwell, J. C., 1892, A treatise on electricity and magnetism [3d ed.]: Oxford Clarendon Press, 2 v. (Reprinted 1954, New York, Dover Pub., 2 v. in 1). 
Nagata, Takesi, 1961, Rock magnetism: Tokyo, Maruzen Co., 350 p.

Nagata, Takesi, and Akimoto, Syun-iti, 1961, Magnetic properties of rockforming ferromagnetic minerals, in Nagata, Takesi, ed., Rock magnetism: Tokyo, Maruzen Co., p. 75-125.

Nagata, Takesi, and Kobayashi, K., 1961, Measuring instruments for magnetic properties of rocks and minerals, in Nagata, Takesi, ed., Rock magnetism: Tokyo, Maruzen Co., p. 40-74.

Nagata, Takesi, and Uyeda, Seiya, 1961, General magnetic properties of rocks, in Nagata, Takesi, ed., Rock magnetism: Tokyo, Maruzen Co., p. 126-146.

Noltimier, H. C., 1965, The time dependent magnetic susceptibility of some red sediments: Univ. Newcastle-upon-Tyne, Newcastle-upon-Tyne, England, Ph.D. thesis, $345 \mathrm{p}$.

1967 Use of the spinner magnetometer for anisotropy measurements, in Collinson, D. W., Creer, K. M., and Runcorn, S. K., ed., Methods in paleomagnetism: New York, Elsevier Publishing Co., p. 399-402.

1971, Magnetic rock cylinders with negligible shape anisotropy: Jour. Geophys. Research, v. 76, no. 17, p. 4035-4037.

Nye, J. F., 1960, Physical properties of crystals-their representation by tensors and matrices: London, Clarendon Press, $322 \mathrm{p}$.

Olsen, Eigil, 1966, Applied magnetism-a study in quantities: New York, Springer-Verlag, 144 p.

Page, C. H., 1973, Ambiguities in the use of unit names: Science, v. 179, p. 873-875.

Page, C. H., and Vigoureux, Paul, eds., 1972, The International System of Units (SI) : U.S. Natl. Bur. Standards Spec. Pub. 330 (1972 ed.), 42 p.

Parasnis, D. S., 1961, Magnetism; from lodestone to polar wandering: London, Hutchinson, $128 \mathrm{p}$.

Parratt, L. G., 1961, Probability and experimental errors in science: New York, John Wiley \& Sons, 255 p.

Porath, Hartmut, Stacey, F. D., and Cheam, A. S., 1966, The choice of specimen shape for magnetic anisotropy measurements on rocks: Earth and Planetary Sci. Letters, v. 1, p. 92.

Post, E. J., 1962, Formal structure of electromagnetics: New York, Interscience Publishers, 204 p.

Rayleigh, J. W. S., 1887, Notes on electricity and magnetism (III. On the behavior of iron and steel under the operation of feeble magnetic forces) : Philos. Mag., v. 23, p. 225-245.

Reilly, W. I., 1972, Use of the International System of Units (SI) in geophysical publications: New Zealand Jour. Geology and Geophysics, v. 15, p. 148-154.

Saaty, T. L., and Bram Joṣeph 1964, Nonlinear mathematics: New York, McGraw-Hill Book Co., 381 p.

Sas, R. K., and Pidduck, F. B., 1947, The metre-kilogram-second system of electrical units: London, Methuen \& Co. 60 p.

Scheid, Francis, 1968, Theory and problems of numerical analysis: New York, McGraw-Hill Book Co., 422 p.

Sharma, P. V., 1966, Rapid computation of magnetic anomalies and demagnetization effects caused by bodies of arbitrary shape: Pure and Appl. Geophysics, v. 64, p. 89-109.

- 1968, Demagnetization effect of a rectangular prism: Geophysics, v. 33 , no. 1, p. 132-134. 
Slater, J. C., and Frank, N. H., 1947, Electromagnetism: New York, McGrawHill Book Co., 240 p.

Smythe, W. R., 1968, Static and dynamic electricity: New York, McGraw-Hill Book Co., 623 p.

Sokolnikoff, I. S., 1964, Tensor analysis-theory and applications to geometry and mechanics of continua: New York, John Wiley \& Sons, 361 p.

Sommerfield, J. W., 1952, Electrodynamics, Translated by E. G. Ramberg. v. 3 of lectures on theoretical physics: New York, Academic Press, 371 p.

Stacey, F. D., 1961, Theory of the magnetic properties of igneous rocks in alternating fields: Philos. Mag., v. 6, no. 67, p. 1241-1260.

Stacey, F. D., Joplin, Germaine, Lindsay, S., 1960, Magnetic anisotropy and fabric of some foliated rocks from S. E. Australia: Pure and Applied Geophysics, v. 47 , p. $30-40$.

Standley, K. J., 1972, Oxide magnetic materials: London, Oxford Univ., Clarendon Press, 254 p.

Stone, D. B., 1963, Anisotropic magnetic susceptibility measurements on a phonolite and on a folded metamorphic rock: Royal Astron. Soc. Geophy. Jour., v. 7, p. 375-390.

1967, An anisotropy meter, in Collinson, D. W., Creer, K. M., and Runcorn, S. K., eds., Methods in paleomagnetism: New York, Elsevier Publishing Co., p. 372-380 (with appendix on estimating the precision of a set of results).

Stoner, E. C., 1934, Magnetism and matter: London, Methuen \& Co., 575 p.

1945, The demagnetizing factors for ellipsoids: Philos. Mag., v. 36, no. 263, p. 803-821.

Stratton, J. A., 1941, Electromagnetic theory: New York, McGraw-Hill Book Co., $615 \mathrm{p}$.

Symon, K. R., 1960, Mechanics: Reading, Mass., Addison-Wesley Pub. Co. $557 \mathrm{p}$.

Tropper, A. M., 1962, Matrix theory for electrical engineers: Reading, Mass., Addison-Wesley Pub. Co., 98 p.

Uspensky, J. V., 1948, Theory of equations: New York, McGraw-Hill Book Co., $353 \mathrm{p}$.

Uyeda, Seiya, Fuller, M. D., Belshé, J. C., and Girdler, R. W., 1963, Anisotropy of magnetic susceptibility of rocks and minerals: Jour. Geophys. Research, v. 68, no. 1, p. 279-291.

Vigoureux, Paul, 1971, Units and standards for electromagnetism: New York, Springer-Verlag, $80 \mathrm{p}$.

Werner, Sture, 1945, Determinations of the magnetic susceptibility of ores and rocks from Swedish iron ore deposits: Stockholm Sveriges Geol. Undersökning, Årsb. 39, no. 5, ser. c, no. 472, 79 p.

Williams, S. R., 1931, Magnetic phenomena: New York, McGraw-Hill Book Co., 230 p.

Wills, A. P., 1931, Vector analysis with an introduction to tensor analysis: New York, Prentice Hall, 285 p.

Wilson, E. B., 1909, Vector analysis-a textbook for the use of students of mathematics and physics based upon the lectures of J. Willard Gibbs: New York, Dover Publishers, 436 p.

Wing-Fatt, Leong, and Stacey, F. D., 1966, Magnetic anisotropy of laboratory materials in which magma flow is simulated: Pure and Appl. Geophysics, v. 64 , p. 78-80.

Zijlstra, H., 1967, Experimental methods in magnetism, Volume IX, Measurement of magnetic quantities: New York, John Wiley \& Sons, 296 p. 